Aus der Klinik für Anaesthesiologie

(Prof. Dr. med. M. Quintel)

im Zentrum Anaesthesiologie-, Rettungs- und Intensivmedizin der Medizinischen Fakultät der Universität Göttingen

\title{
Evaluation der Effektivität eines neuen konduktiven Wärmesystems zur Prävention perioperativer Hypothermie in der Hals-Nasen-Ohren-Chirurgie
}

\author{
INAUGURAL-DISSERTATION \\ zur Erlangung des Doktorgrades \\ der Medizinischen Fakultät der \\ Georg-August-Universität zu Göttingen \\ vorgelegt von \\ Christine Maria Blecken \\ aus Gifhorn
}

Göttingen 2015 
De k a n:

I. Berichterstatter:

II. Berichterstatter:
Prof. Dr. rer. nat. H. K. Kroemer

Prof. Dr. med. A. Bräuer

PD Dr. med. Dr. rer. nat. A. Meyer

Tag der mündlichen Prüfung: $\quad$ 01.12.2015 


\section{Inhalt}

II. ABKÜRZUNGEN, ABBILDUNGEN UND TABELLEN ................................

II.1 Liste der verwendeten Abkürzungen ................................................

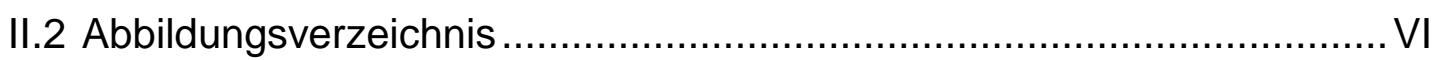

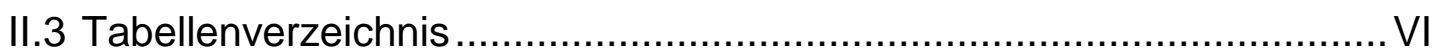

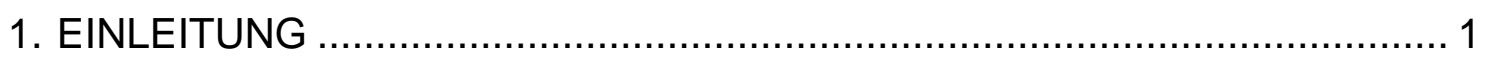

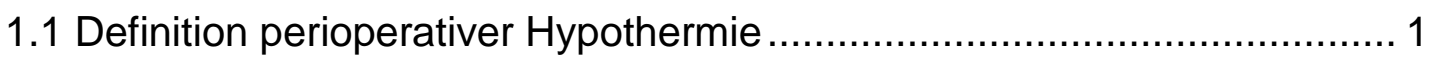

1.2 Bedeutung perioperativer Hypothermie ............................................... 1

1.2.1 Häufigkeit perioperativer Hypothermie.......................................... 1

1.2.2 Risiken und Auswirkungen perioperativer Hypothermie ................ 2

1.2.2.1 Kardiovaskuläre Komplikationen .................................... 2

1.2.2.2 Beeinträchtigung der Gerinnung .................................... 3

1.2.2.3 Wundinfektionen ....................................................... 4

1.2.2.4 Beeinflussung der Pharmakokinetik ................................. 5

1.2.2.5 Postoperatives Kältezittern (Shivering) ………………..... 5

1.2.2.6 Bedeutung für den Aufwachraum ...................................... 6

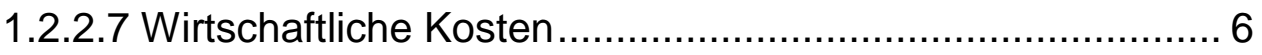

1.3 Entstehung perioperativer Hypothermie ........................................... 7

1.3.1 Normale Thermoregulation bei Abkühlung …….......................... 7

1.3.2 Bedeutung der Allgemeinanästhesie ........................................... 8

1.3.3 Auswirkungen der Allgemeinanästhesie als Phasenmodell ............ 8

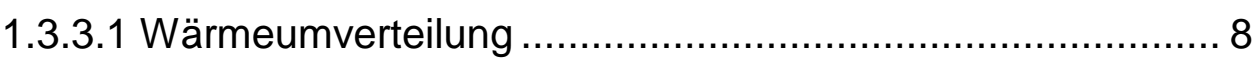

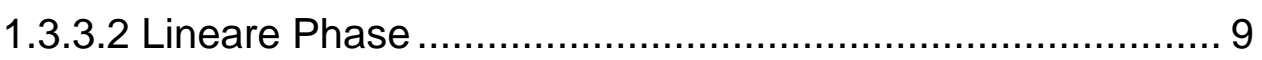

1.3.3.3 Plateauphase .......................................................... 10

1.4 Physikalische Grundlagen des Wärmetransfers ............................... 10

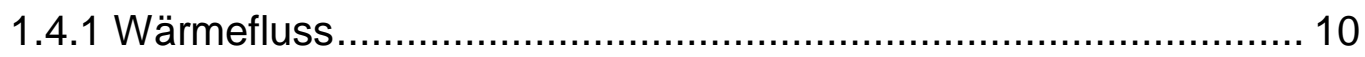

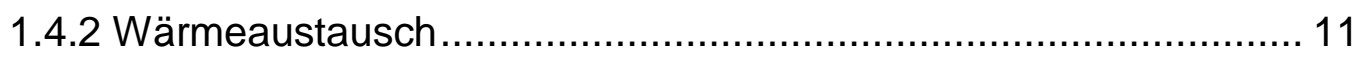

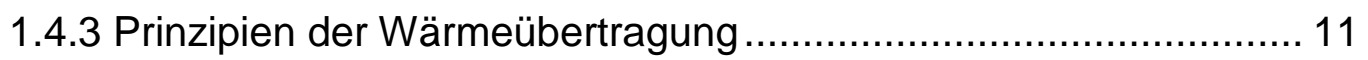

1.4.3.1 Konduktion ................................................................. 11

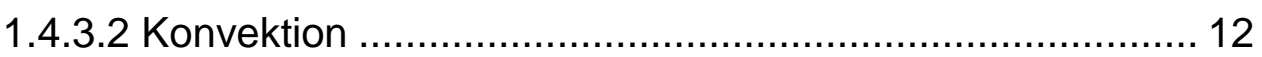

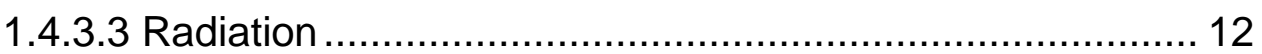

1.4.3.4 Evaporation ......................................................... 12

1.5 Prävention perioperativer Hypothermie ........................................... 13

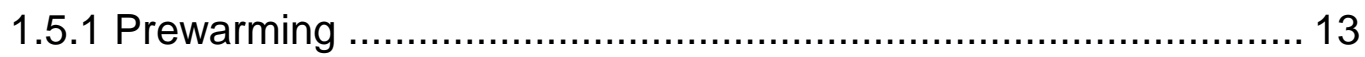

1.5.2 Intraoperative Wärmeprotektionsverfahren................................. 14

1.5.2.1 Wärmeprotektion durch Erhöhung der Raumtemperatur... 14

1.5.2.2 Wärmeprotektion durch Isolation..................................... 14

1.5.2.3 Wärmeprotektion durch Heizmatten unter dem Rücken.... 15 
1.5.2.4 Wärmeprotektion durch auf den Körper gelegte

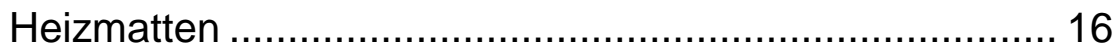

1.5.2.5 Wärmeprotektion durch konvektive Luftwärmer ............... 16

1.5.2.6 Sonstige Verfahren ..................................................... 17

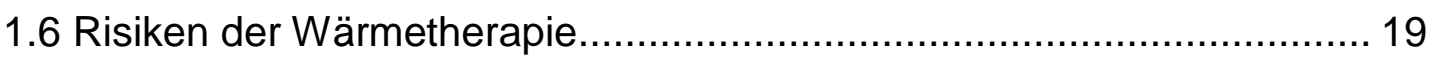

1.6.1 Auftreten von Verbrennungen ................................................ 19

1.6.2 Störung des Luftstroms in Laminar-Airflow-Sälen......................... 19

1.6.3 Nutzen-Risiko-Relation der Prävention von Hypothermie ............. 20

1.7 Inzidenz perioperativer Hypothermie bei HNO-Patienten ..................... 20

1.7.1 Prävention und Risiken bei HNO-chirurgischen Eingriffen............ 20

1.8 Das neue konduktive Wärmesystem Barrier ${ }^{\circledR}$ Easywarm ${ }^{\circledR}$.................... 21

1.8.1 Möglichkeiten des perioperativen Wärmemanagements ............. 21

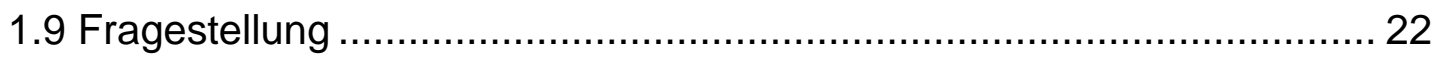

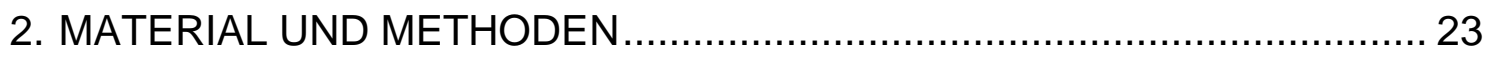

2.1 Randomisierung und Patientenrekrutierung ....................................... 23

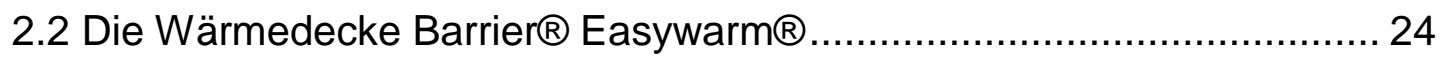

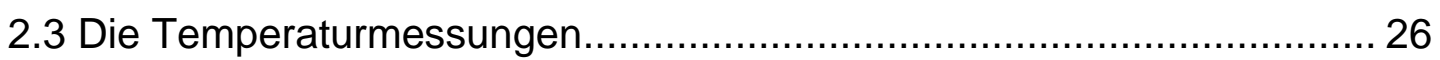

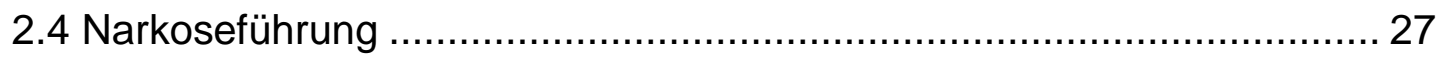

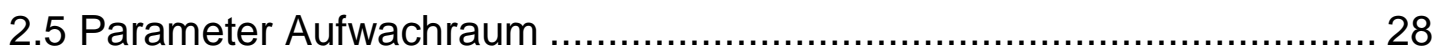

2.6 Fallzahlplanung und statistische Auswertung ...................................... 28

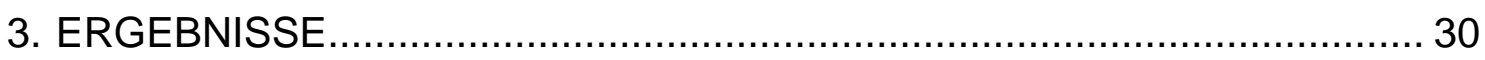

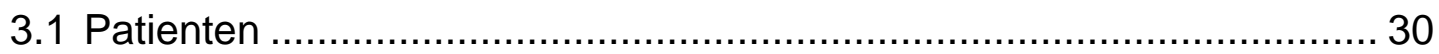

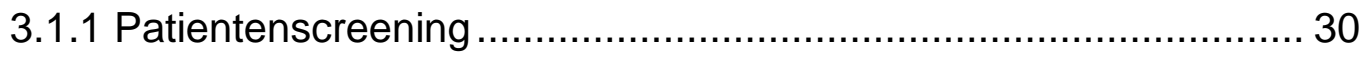

3.1.2 Präoperativ ausgeschlossene Patienten.................................... 31

3.1.3 Postoperativ ausgeschlossene Patienten ................................... 31

3.1.4 Eingeschlossene Patienten .................................................... 32

3.1.4.1 Vergleich der biometrischen Daten ................................ 33

3.1.4.2 Vergleich der Operations- und Narkosedauer ................... 34

3.1.4.3 Vergleich der Vorwärmdauer in der Interventionsgruppe .. 34

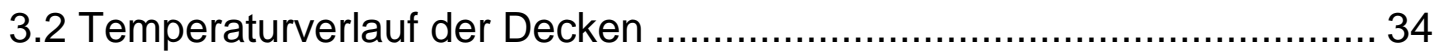

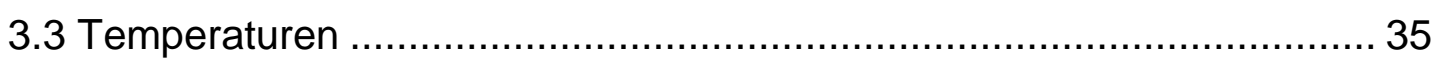

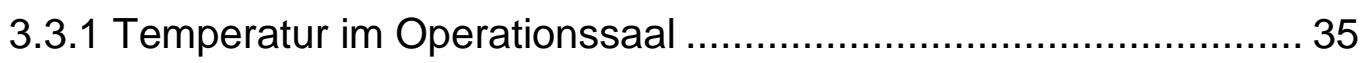

3.3.2 Präoperative Temperaturen ................................................... 35

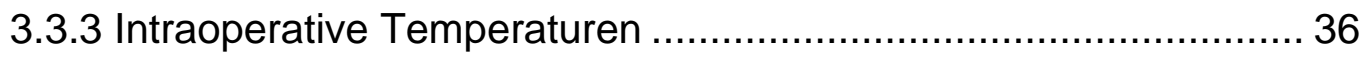

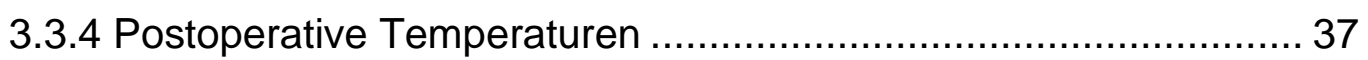

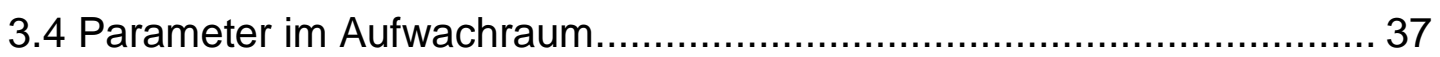

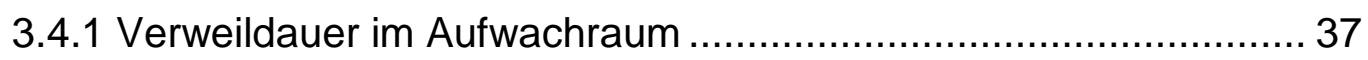

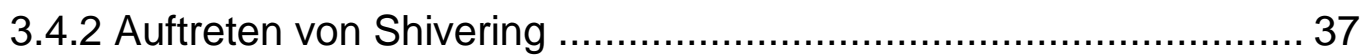




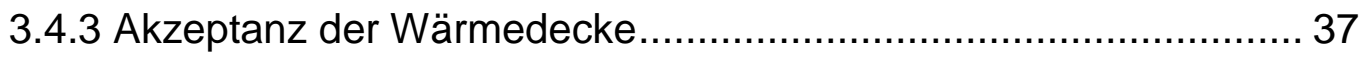

3.4.4 Nebenwirkungen der Wärmetherapie ......................................... 38

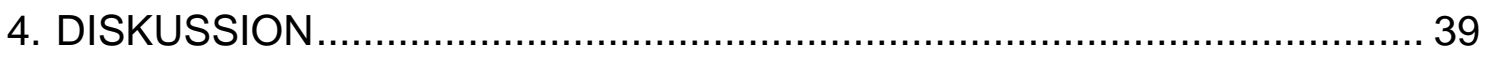

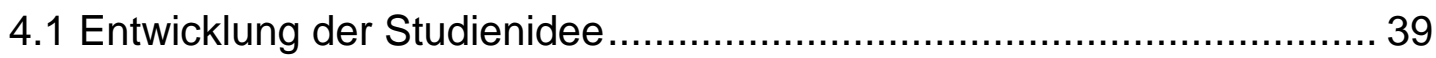

4.1.1 Potentielle Vorteile der Wärmedecke Barrier ${ }^{\circledR}$ Easywarm $\AA$......... 40

4.1.2 Potentielle Nachteile der Wärmedecke Barrier ${ }^{\circledR}$ Easywarm ${ }^{\circledR}$...... 40

4.2 Interpretation der Ergebnisse....................................................... 41

4.2.1 Klinische Interpretation der Ergebnisse .................................. 41

4.2.2 Erklärung der Ergebnisse durch die Modelluntersuchung ........... 44

4.3 Kritische Würdigung der Ergebnisse ................................................. 45

4.3.1 Diskussion der Wärmedecke Barrier ${ }^{\circledR}$ Easywarm ${ }^{\circledR}$ im Vergleich zu anderen konduktiven Wärmedecken ...................................... 45

4.3.2 Diskussion der Wärmedecke Barrier ${ }^{\circledR}$ Easywarm $\circledast$ im Vergleich zu konvektiver Luftwärmung.

4.3.3 Diskussion der Wärmedecke Barrier ${ }^{\circledR}$ Easywarm ${ }^{\circledR}$ im Vergleich zu verschiedenen Isolationsmaterialien 48

4.3.4 Diskussion von Wirtschaftlichkeit und Patientenkomfort der Wärmedecke Barrier ${ }^{\circledR}$ Easywarm $\AA$............................................ 49

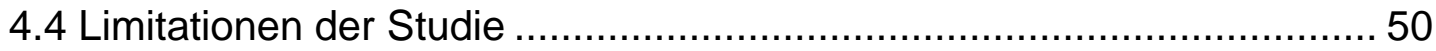

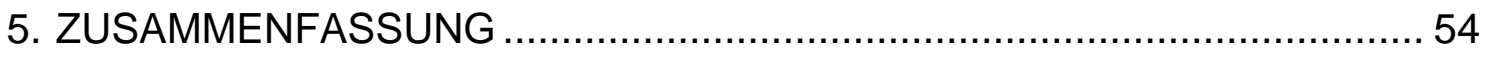

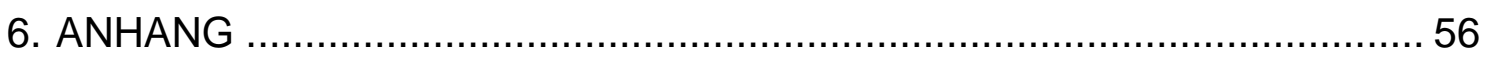

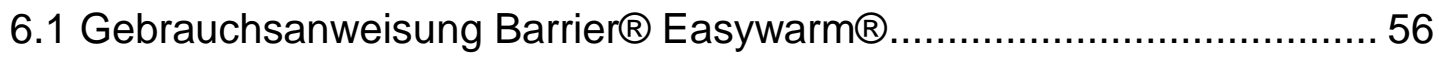

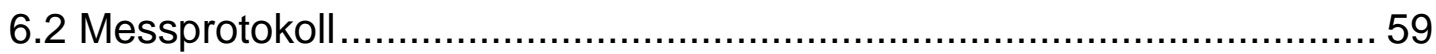

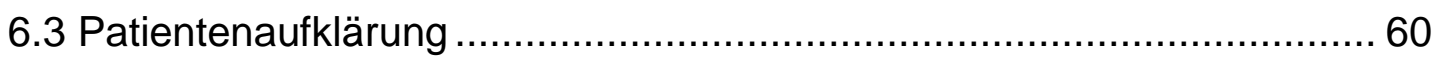

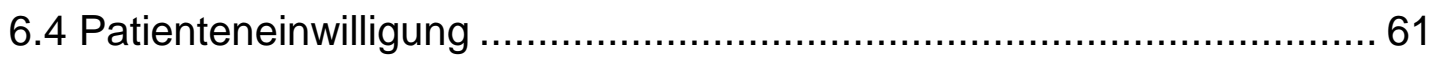

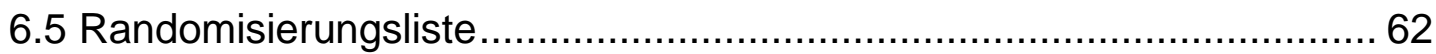

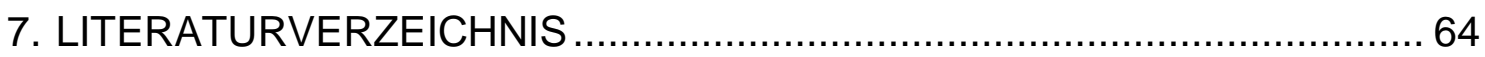




\section{Abkürzungen, Abbildungen und Tabellen}

\section{II.1 Liste der verwendeten Abkürzungen}

A

ANOVA

ASA

BMI

${ }^{\circ} \mathrm{C}$

clo

$\mathrm{cm}$

ca.

$\$$

$€$

ggf.

$\mathrm{HNO}$

$\mathrm{h}$

IEC

$£$

$\mathrm{m}$

MAC

$\mathrm{mg}$

$\mu \mathrm{g}$

$\min$

$\mathrm{m} / \mathrm{w}$

MW

NICE

OP

PTT

Q

$\dot{Q}$

SD

SI

$\mathrm{t}$

$\Delta \mathrm{T}$

W

z. B.
Fläche

analysis of variance

Score der American Society of Anesthesiologists

Body Mass Index

Grad Celsius

Einheit der Isolation

Zentimeter

circa

Amerikanischer Dollar

Euro

gegebenenfalls

Hals-Nasen-Ohrenheilkunde

Wärmeaustauschkoeffizient

International Electrotechnical Commission

Britisches Pfund

Meter

minimale alveoläre Konzentration

Milligramm

Mikrogramm

Minuten

männlich/weiblich

arithmetischer Mittelwert

National Institute for Health and Clinical Excellence

Operation

partielle Thromboplastinzeit

Wärmemenge

Wärmeaustausch

Standardabweichung

Système international d'unités

Zeit

Temperaturgradient

Watt

zum Beispiel 


\section{II.2 Abbildungsverzeichnis}

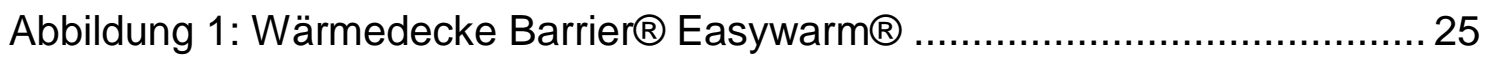

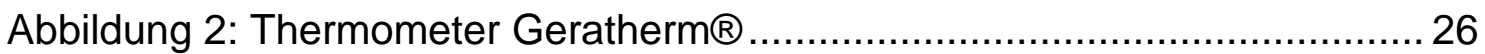

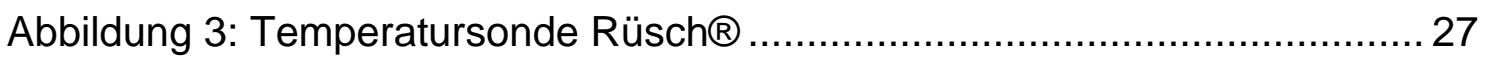

Abbildung 4: Flussdiagramm Patientenkollektiv ......................................... 30

Abbildung 5: Vier exemplarische Messungen der Temperaturentwicklung nach Aktivierung der Decken ............................................................... 35

Abbildung 6: Mittelwerte und Standardabweichungen der perioperativen

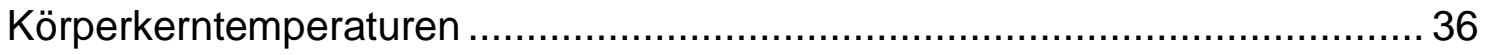

\section{II.3 Tabellenverzeichnis}

Tabelle 1: Patientendaten und perioperative Verlaufsdaten 


\section{EINLEITUNG}

\subsection{Definition perioperativer Hypothermie}

Perioperative Hypothermie ist definiert durch einen Abfall der Körperkerntemperatur unter $36{ }^{\circ} \mathrm{C}$ (NICE: National Institute for Health and Clinical Excellence 2008). Sessler stellt 2001 fest, dass der Begriff „milde Hypothermie“ in der Literatur uneinheitlich gebraucht wird (Sessler 2001). Er definiert in seiner Übersichtsarbeit "Complications and Treatment of Mild Hypothermia“ eine Körperkerntemperatur zwischen $34^{\circ} \mathrm{C}$ und $36^{\circ} \mathrm{C}$ als milde Hypothermie (Sessler 2001). In der Mehrheit der Studien wird der Schwellenwert von $36^{\circ} \mathrm{C}$ bereits vor Publikation der Guidelines des National Institute auf Clinical Excellence im Jahr 2008 verwendet (Kongsayreepong et al. 2003). Begründet wurde dies von Kongsayreepong et al. (2003) mit dem Verweis auf klinische Untersuchungen, die Hinweise darauf geben, dass bereits Temperaturen unter $36^{\circ} \mathrm{C}$ in der postoperativen Phase physiologische Veränderungen bedingen, die zu einem schlechteren Gesundheitszustand des Patienten führen können (Slotman et al. 1985). In einigen älteren und wenigen neuen Studien wird dagegen ein Schwellenwert von $35^{\circ} \mathrm{C}$ festgelegt (Abelha et al. 2005) oder eine Körperkerntemperatur unter $35^{\circ} \mathrm{C}$ als schwere Hypothermie bezeichnet (Karalapillai et al. 2009).

\subsection{Bedeutung perioperativer Hypothermie}

\subsubsection{Häufigkeit perioperativer Hypothermie}

Das Auftreten perioperativer Hypothermie ist vor allem bei Patienten, die sich einer Allgemeinanästhesie unterziehen, eine häufige Komplikation. In mehreren Studien zeigt sich, dass bei Aufnahme auf die Intensivstation mehr als $50 \%$ der Patienten nach nicht-kardiochirurgischen Eingriffen hypotherm sind. Dies bestätigen Studien von Slotman et al. (1985) mit 53 \%, von Kongsayreepong et al. (2003) mit 57 \% sowie von Karalapillai und Story (2008) mit 55 \% postoperativ hypothermen nicht-kardiochirurgischen Patienten. Die größte und aktuellste Studie in diesem Patientenkollektiv an über 50.000 Patienten demonstriert eine Inzidenz von $46 \%$ postoperativer Hypothermie bei Aufnahme auf die Intensivstation (Karalapillai et al. 2013). Bei einem Schwellenwert von $35^{\circ} \mathrm{C}$ beschreiben Abelha et al. (2005) eine Inzidenz von $58 \%$ hypothermen Patienten bei Aufnahme auf die Intensivstation. Diese hohen Inzidenzen von Patienten mit Temperaturen unter $35^{\circ} \mathrm{C}$ werden in anderen Studien nicht gefunden. So zeigen Untersuchungen von Kongsayreepong et al. (2003) Inzidenzen von $28 \%$ und von Karalapillai und Story (2008) sogar nur Inzidenzen von $12 \%$ von Patienten mit 
Temperaturen unter $35^{\circ} \mathrm{C}$. Demgegenüber weisen Patienten nach kardiochirurgischen Eingriffen bei Aufnahme auf die Intensivstation mit circa $30 \%$ eine geringere Hypothermieinzidenz auf. In den Studien von Insler et al. (2000) und Karalapillai et al. (2009) an über 5.000 Patienten haben $28 \%$ bzw. $31 \%$ der Patienten bei Aufnahme auf die Intensivstation eine Körperkerntemperatur von unter $36{ }^{\circ} \mathrm{C}$. Der Unterschied zwischen dem vermehrten Auftreten von Hypothermie bei nicht-kardiochirurgischen gegenüber kardiochirurgischen Patienten ist statistisch signifikant (Karalapillai und Story 2008) und wegen der assoziierten Risiken und unerwünschten Auswirkungen auch klinisch relevant.

\subsubsection{Risiken und Auswirkungen perioperativer Hypothermie}

In mehreren Studien wird gezeigt, dass perioperative Hypothermie mit einem erhöhten Risiko für verschiedene relevante intra- und postoperative Komplikationen verbunden ist (Sessler 2001).

\subsubsection{Kardiovaskuläre Komplikationen}

Von großer Bedeutung sind akute kardiale Erkrankungen wie Myokardinfarkte und Myokardischämien, akute Herzinsuffizienz und Rhythmusstörungen, da sie zu den häufigsten Ursachen tödlicher Komplikationen im direkten perioperativen Zeitfenster zählen (Buhre und Rossaint 2003). Bereits bei einem Abfall der Körperkerntemperatur um $1,3^{\circ} \mathrm{C}$ erhöht sich die Gesamtrate ventrikulärer Tachykardien, Myokardischämien und tödlicher kardialer Ereignisse postoperativ auf das Zwei- bis Dreifache verglichen mit normothermen Patienten (Frank et al. 1997a). Diese prospektive Studie von Frank et al. (1997a) wurde an Patienten mit einem hohen kardialen Risikoprofil durchgeführt und zeigt, dass durch Einhaltung von Normothermie das relative Risiko für tödliche Myokardischämien um $55 \%$ reduziert werden kann. Bei alleiniger Betrachtung der tödlichen kardialen Ereignisse treten diese Komplikationen bei hypothermen Patienten postoperativ mehr als vierfach häufiger auf $(1,4 \%$ versus $6,3 \%, p=0,02)$ (Frank et al. 1997a). Obwohl in dieser Untersuchung auch die Häufigkeit des Kältezitterns in der Gruppe der hypothermen Patienten erhöht ist, hat das Auftreten von postoperativem Shivering keinen Einfluss auf die Häufung kardialer Ereignisse (Frank et al. 1993, Frank et al. 1997a). Entgegen früheren Untersuchungen an relativ jungen und gesunden Patienten, die einen Anstieg der metabolischen Rate durch Kältezittern um $400 \%$ beschreiben (Bay et al. 1968), finden Frank et al. (1995a) bei Patienten über 60 Jahren lediglich einen Anstieg des Gesamtsauerstoffverbrauchs um $40 \%$. Sie schlussfolgern daraus, dass die Erhöhung der Hypother- 
mie assoziierten kardialen Ereignisse nicht durch einen gesteigerten Sauerstoffverbrauch während des Zitterns bedingt ist. Dagegen scheint der Anstieg der Plasmakonzentration von Noradrenalin und die damit einhergehende Hypertension die Rate postoperativer kardialer Komplikationen zu beeinflussen. So zeigen Studien an Probanden (Frank et al. 1997b) und Patienten (Frank et al. 1995b) eine durch Kälte induzierte Erhöhung der Plasmaspiegel von Noradrenalin, die eine vermehrte periphere Vasokonstriktion sowie konsekutive Hypertension vermitteln.

\subsubsection{Beeinträchtigung der Gerinnung}

Die Blutgerinnung wird durch den Einfluss von Hypothermie auf verschiedenen Ebenen derart gestört, dass ein erhöhter Blutverlust und Transfusionsbedarf resultiert. Bei Patienten, die einen Hüftgelenkersatz erhalten haben, führt ein Abfall der Körperkerntemperatur um $1,6^{\circ} \mathrm{C}$ zu einer Steigerung des Blutverlustes um $500 \mathrm{ml}$ und damit zu einer relativen Erhöhung um $30 \%$ sowie zu einem signifikanten Anstieg des Transfusionsbedarfs (Schmied et al. 1996). Auch in vielen weiteren Studien mit allgemeinchirurgischen (Bock et al. 1998, Kurz et al. 1996) oder kardiochirurgischen Operationen (Hofer et al. 2005) weisen hypotherme Patienten einen signifikant höheren Blutverlust und Transfusionsbedarf auf. Durch forcierte Wärmemaßnahmen kann der Blutverlust bei Hüftoperationen um $200 \mathrm{ml}$ reduziert werden, wenn Normothermie von $36,5^{\circ} \mathrm{C}$ verglichen mit $36,0^{\circ} \mathrm{C}$ eingehalten wird (Winkler et al. 2000). Dagegen kann in einer anderen Studie der Einfluss von Hypothermie auf einen erhöhten Blutverlust bei Hüftoperationen nicht nachgewiesen werden (Johansson et al. 1999). In einer aktuellen Metaanalyse wurde auf Basis von 14 prospektiven Studien die Frage nach dem Einfluss von Hypothermie auf einen gesteigerten Blutverlust sowie auf Basis von zehn Studien der Einfluss von Hypothermie auf einen erhöhten Transfusionsbedarf untersucht (Rajagopalan et al. 2008). Hier zeigt sich, dass bereits milde Hypothermie von weniger als $1{ }^{\circ} \mathrm{C}$ im Vergleich zu Normothermie mit einem um $16 \%$ erhöhten Blutverlust sowie einem um 22 \% erhöhten relativen Risiko für die Gabe von Bluttransfusionen verbunden ist.

Die durch Hypothermie hervorgerufene Gerinnungsstörung resultiert aus der Beeinträchtigung von Enzymfunktionen, die sowohl zu Störungen der Thrombozytenfunktion als auch der plasmatischen Gerinnung führt (Sessler 2001). So bewirken geringere Temperaturen eine Reduktion der Enzymaktivität der Thromboxansynthetase, wodurch die Thromboxan- $\mathrm{A}_{2}$-Bildung vermindert ist und dadurch eine reversible Störung der Adhäsions- und Aggregationsfunktionen der Throm- 
bozyten bedingt wird (Valeri et al. 1987, Valeri et al. 1992). Aber auch die Funktion der plasmatischen Gerinnung wird durch Hypothermie ebenfalls beeinträchtigt. Zu beachten ist, dass Untersuchungsbedingungen von $37^{\circ} \mathrm{C}$ im Routinelabor den Einfluss der Temperatur auf die Gerinnungswerte verdecken. Den Effekt von Hypothermie auf die plasmatische Gerinnung wird in Untersuchungen der Temperaturabhängigkeit der partiellen Thromboplastinzeit (PTT) demonstriert (Rohrer und Natale 1992). Bei Temperaturen im normothermen Bereich von $37^{\circ} \mathrm{C}$ beträgt die PTT 36 Sekunden. Sie verlängert sich bei einer Abkühlung auf $34^{\circ} \mathrm{C}$ auf 39,4 Sekunden und beträgt bei $31^{\circ} \mathrm{C}$ sogar schon 46,1 Sekunden.

\subsubsection{Wundinfektionen}

Eine weitere häufige und bedeutende Komplikation im postoperativen Verlauf ist das Auftreten von Wundinfektionen und Wundheilungsstörungen. Bereits bei einem Abfall der Körperkerntemperatur auf $34,7^{\circ} \mathrm{C}$ erhöht sich die Anzahl an Wundinfektionen auf das Dreifache und der Krankenhausaufenthalt verlängert sich von 12,1 auf 14,7 Tage und damit relativ um mehr als $20 \%$ wie Kurz et al. (1996) an 200 Patienten mit kolorektalen Operationen zeigen. Bemerkenswert ist in dieser Studie, dass die Liegedauer in der Hypothermiegruppe um 1,7 Tage auch statistisch signifikant verlängert ist, wenn lediglich die nicht infizierten Patienten analysiert werden. Ein Grund hierfür könnte das vermehrte Auftreten von Wundheilungsstörungen sein, da Hypothermie zu einer verringerten Kollagenbildung im Wundgebiet führt (Kurz et al. 1996).

Mehrere Faktoren beeinträchtigen direkt und indirekt die immunologische Abwehr des Körpers durch Hypothermie. Zum einen werden die Lymphozyten und neutrophilen Granulozyten direkt in ihrer Funktion geschwächt. Daraus resultiert eine reduzierte Antikörperbildung, geringere Chemotaxis (van Oss et al. 1980) und verminderte Phagozytosekapazität (Wenisch et al. 1996). Zum anderen bewirkt Hypothermie eine thermoregulatorische Vasokonstriktion, wodurch der Sauerstoffpartialdruck im subkutanen Gewebe sinkt (Sheffield et al. 1996). Dies führt zu einer indirekten Beeinträchtigung der immunologischen Abwehr durch Hypothermie. Durch den gesunkenen Sauerstoffpartialdruck steht den neutrophilen Granulozyten weniger Sauerstoff zur Bildung von Sauerstoffradikalen zur Verfügung, wodurch das Abtöten phagozytierter Keime vermindert ist (Hohn et al. 1976). Insgesamt zeigt sich zudem eine Korrelation zwischen einem sinkenden subkutanen Sauerstoffpartialdruck und einem Anstieg von Wundinfektionen (Hopf et al. 1997). 


\subsubsection{Beeinflussung der Pharmakokinetik}

Die Wirkdauer vieler Anästhetika ist unter anderem durch einen Metabolismus durch Enzyme bedingt (Sessler 2001). Diese sind jedoch in ihrer Funktion von der Temperatur abhängig und werden daher in ihrer Kinetik durch Hypothermie beeinflusst (Sessler 2001). Allerdings ist die Pharmakokinetik gängiger Anästhetika bei Hypothermie nur in wenigen Studien beim Menschen untersucht worden. Bei kontinuierlicher Zufuhr von Propofol finden sich bei hypothermen Patienten um 28 \% höhere Plasmaspiegel (Leslie et al. 1995). Bei kontinuierlicher Fentanylapplikation bei hypothermen Schweinen mit einer Temperatur von $32^{\circ} \mathrm{C}$ wird ein Anstieg der Plasmaspiegel um $25 \%$ beschrieben, welcher auch nach Wiedererwärmung über mehrere Stunden erhöht bleibt (Fritz et al. 2005). Bei Muskelrelaxanzien werden für Vecuronium (Heier et al. 1991), Atracurium (Leslie et al. 1995) und Rocuronium (Smeulers et al. 1995) Verlängerungen der Wirkdauer nachgewiesen. Diese ist bei Vecuronium mit einer Verdopplung der Wirkdauer bei einem Temperaturabfall um $2{ }^{\circ} \mathrm{C}$ am stärksten ausgeprägt. Bei Atracurium verlängert sich die Wirkdauer bei einem Abfall der Körperkerntemperatur um $3{ }^{\circ} \mathrm{C}$ dagegen nur um $60 \%$. Bei volatilen Anästhetika erniedrigt sich die minimale alveoläre Konzentration (MAC) um $5 \%$ pro $1^{\circ} \mathrm{C}$ Temperaturabfall (Liu et al. 2001). Allerdings zeigt sich in einer aktuellen Studie an Schweinen kein Einfluss der Hypothermie auf die MAC bei Xenon im Vergleich zu Sevofluran (Liu et al. 2013).

\subsubsection{Postoperatives Kältezittern (Shivering)}

Postoperatives Shivering kann sowohl kälteinduziert als Kältezittern sowie auch nicht-thermoregulatorisch bedingt, z. B. durch Schmerz, auftreten (Horn et al. 1999). Shivering ist eine häufige Komplikation bei hypothermen Patienten und tritt im Vergleich zu normothermen Patienten signifikant häufiger auf (Horn et al. 1998). Dabei wird die Größenordnung für das Auftreten von Shivering bei hypothermen Patienten nach einer Allgemeinanästhesie mit volatilen Anästhetika in der Literatur mit bis zu 60 \% angegeben (Horn et al. 1997). Das Kältezittern ist gekennzeichnet durch eine unwillkürliche, periodische Muskelaktivität, die der Steigerung der Wärmeproduktion dient (Sessler et al. 1991b). Dennoch erhöht Shivering bei alten Patienten den Gesamtsauerstoffverbrauch lediglich um $40 \%$ (Frank et al. 1995a). Dabei steigt der erhöhte Sauerstoffverbrauch durch Kältezittern proportional zum Abfall der Körperkerntemperatur an (Just et al. 1992). Diese Beobachtung berichten auch Just et al. (1992), die postoperativ einen Anstieg des Sauerstoffverbrauchs sowie der Kohlenstoffdioxidproduktion auf das Zwei- bis Dreifache bei hypothermen Patienten mittleren Alters beobachten. Zu 
bemerken ist, dass Shivering bei Patienten im höheren Lebensalter seltener auftritt (Frank et al. 1995a) und selber durch Hypoxie inhibiert wird (Gautier et al. 1987). Daher ist anzunehmen, dass Kältezittern selber nicht die maßgebliche Ursache der mit der Hypothermie assoziierten kardialen Komplikationen ist.

In der postoperativen Phase führt das vermehrte Auftreten von Kältezittern zu einem erhöhten Diskomfort bei den Patienten (Kurz et al. 1995b). Neben einer Beeinträchtigung des Wohlbefindens kann Kältezittern durch Dehnung der Operationswunden aber auch zu vermehrten Schmerzereignissen führen (De Witte und Sessler 2002).

\subsubsection{Bedeutung für den Aufwachraum}

Die Auswirkungen perioperativer Hypothermie beeinflussen sowohl das Wohlbefinden des Patienten als auch dessen Verweildauer im Aufwachraum. So beklagen hypotherme Patienten vermehrtes Kältegefühl und benötigen bis zu vier Stunden um ihre Körperkerntemperatur zu normalisieren (Kurz et al. 1995b). In einer Studie von Lenhardt et al. (1997) werden die Konsequenzen von Hypothermie auf die Verweildauer im Aufwachraum untersucht. So wird gezeigt, dass hypotherme Patienten 40 Minuten später aus dem Aufwachraum entlassen werden, selbst wenn die Körperkerntemperatur bei Entlassung nicht als Entscheidungsmerkmal herangezogen wird. Ist diese ein Entlassungskriterium gewesen, beträgt die Differenz zu normothermen Patienten sogar 90 Minuten (Lenhardt et al. 1997). Ähnliche Daten finden auch Bock et al. (1998), die eine um zwei Stunden verlängerte Aufenthaltsdauer im Aufwachraum bei hypothermen Patienten dokumentieren.

\subsubsection{Wirtschaftliche Kosten}

Bei der Betrachtung der wirtschaftlichen Aufwendungen stehen den Kosten des perioperativen Temperaturmanagements die Kosten der durch Hypothermie bedingten Komplikationen gegenüber. Die Kosten für das Temperaturmonitoring setzen sich aus der Anschaffung des Messmoduls und der Temperatursonden, z. B. für einen Blasenkatheter oder für eine Ösophagussonde als Einmalartikel mit 8 bis $10 €$, zusammen. Dazu entstehen Kosten für aktive Wärmetherapie in Abhängigkeit von den eingesetzten Maßnahmen. Eine effektive und häufig verwendete Form der Wärmezufuhr sind konvektive Luftwärmer, wobei Kosten für die Anschaffung und Wartung des Gebläsegerätes (ca. $2000 €$ ) sowie der verwendeten Wärmedecke ( $a b 8 €$ ) entstehen (Ammann et al. 2004). 
Demgegenüber stehen die Kosten, die in Folge der Behandlung der durch Hypothermie bedingten Komplikationen entstehen. Dazu zählen sowohl direkte Kosten beispielsweise für Erythrozytenkonzentrate aufgrund eines erhöhten Transfusionsbedarfes (Rajagopalan et al. 2008). Aber auch die erhöhte Rate an Infektionen (Kurz et al. 1996), Myokardinfarkten (Frank et al. 1997a) und anderen Komplikationen kann zu einem verlängerten Krankenhaus- und Intensivaufenthalt (Kurz et al. 1996, Slotman et al. 1985) mit den damit verbundenen Kosten durch notwendige Therapien, Untersuchungen und die verlängerte Hospitalisierung führen. Daneben führt Hypothermie selber unabhängig vom Auftreten weiterer Komplikationen zu einem verlängerten Krankenhausaufenthalt (Kurz et al. 1996) und ist häufig Ursache ungeplanter Aufnahmen auf die Intensivstation. Dabei wird in $45 \%$ der vermeidbaren, Anästhesie bedingten Komplikationen Hypothermie als Ursache der ungeplanten Aufnahmen auf die Intensivstation angegeben (Bauer et al. 2007). In einer Studie von Bock et al. (1998) an Patienten mit großen allgemeinchirurgischen Operationen wird gezeigt, dass die Kosten um 24 $\%$ niedriger sind, wenn die Patienten mittels Temperatur- und Wärmemanagement normotherm bleiben. Die höheren Kosten bei den hypothermen Patienten, die nicht über konvektive Luftwärmer gewärmt werden, setzen sich in dieser Studie vor allem durch die deutlich verlängerte Liegedauer im Aufwachraum sowie den erhöhten Transfusionsbedarf zusammen (Bock et al. 1998). Ausführlich werden die Kosten und Nutzen durch relative Risikoreduktion in Bezug auf das Ausmaß des Wärmemanagements in den NICE Guidelines diskutiert. Insgesamt wird dort festgestellt, dass das Einhalten von Normothermie bei alten Patienten mit ausgedehnten Eingriffen zu dem größten Nettonutzen führt (NICE 2008).

\subsection{Entstehung perioperativer Hypothermie}

\subsubsection{Normale Thermoregulation bei Abkühlung}

Die Körperkerntemperatur wird trotz wechselnder Umgebungstemperaturen in einem engen Bereich von 36,5 bis $37,5^{\circ} \mathrm{C}$ reguliert (Sessler 2008). Dabei toleriert das thermoregulatorische System des Körpers eine Spannweite von 0,2 bis $0,4^{\circ} \mathrm{C}$ Abweichung von der normalen Körperkerntemperatur, bevor zentrale Regelprozesse in Gang gesetzt werden (Lopez et al. 1994). Bei Abkühlung führen diese zu einer thermoregulatorischen Vasokonstriktion, um die Körperkerntemperatur konstant zu halten. Dieser Mechanismus findet an den arteriovenösen Shunts der Akren statt, welche in einer thermisch ausgeglichenen Umgebung vasodilatiert sind und darüber die Abgabe von Wärme an die Umwelt ermöglichen. Dagegen wird bei kälteren Umgebungstemperaturen aus sympathischen 
Nervenfasern Noradrenalin abgegeben, welches über $\alpha_{1}$-Rezeptoren eine Vasokonstriktion auslöst. Diese Gefäßengstellung führt zu einer Reduktion der Hautdurchblutung und in der Folge zum Absinken der Hauttemperatur. Bei einer Abkühlung der Haut reduziert sich die Temperaturdifferenz zur Umwelt und somit verringern sich auch die Wärmeverluste über die Körperperipherie in einer kalten Umgebung (Sessler 2008).

Des Weiteren erfolgt eine Umverteilung des venösen Rückstroms aus der Körperperipherie von den oberflächlichen zu den tiefen Venen (Bazett et al. 1948). Diese werden von den anliegenden Arterien begleitet, wodurch ein Austausch von Wärme über den gegenläufigen Blutfluss im sogenannten Gegenstromprinzip ermöglicht wird. Dabei fließt kälteres Blut in die Körperperipherie und wärmeres Blut zum Körperkern zurück woraus eine funktionelle Trennung von Körperkern und Körperschale resultiert (Sessler 2009).

Sind diese Maßnahmen der thermoregulatorischen Vasokonstriktion nicht ausreichend um die Körperkerntemperatur konstant zu halten, versucht der Körper die Wärmeproduktion durch Kältezittern zu steigern. Dabei liegt die Schwelle, bei der Kältezittern ausgelöst wird, $1^{\circ} \mathrm{C}$ unter der Schwelle für thermoregulatorische Vasokonstriktion (Lopez et al. 1994).

\subsubsection{Bedeutung der Allgemeinanästhesie}

Patienten sind im perioperativen Bereich stark für die Entwicklung einer Hypothermie gefährdet. Dazu tragen sowohl Umgebungsvariablen wie ein kühler Operationssaal mit laminarem Luftstrom als auch die Veränderungen der Thermoregulationsmechanismen durch die Allgemeinanästhesie bei (Sessler 1997).

\subsubsection{Auswirkungen der Allgemeinanästhesie als Phasenmodell}

Die während einer Allgemeinanästhesie auftretende Hypothermie verläuft in drei charakteristischen Phasen, welche durch verschiedene Ursachen bedingt sind.

\subsubsection{Wärmeumverteilung}

Nach Einleitung der Allgemeinanästhesie kommt es innerhalb der ersten Stunde zu einem schnellen Abfall der Körperkerntemperatur. In dieser Phase beruht der Temperaturabfall auf einer Umverteilung des Blutes aus dem warmen Körperkern in die kältere Körperperipherie. Während die Körperkerntemperatur vor Narkoseeinleitung eng reguliert und konstant gehalten wird, ist die Temperatur der Körperperipherie von den Umgebungstemperaturen abhängig. Dabei resultiert ein normaler Temperaturgradient von 2 bis $4{ }^{\circ} \mathrm{C}$ zwischen der Temperatur des 
Körperkerns und der Hautperipherie, welcher durch die thermoregulatorische Vasokonstriktion etabliert wird (Matsukawa et al. 1995b). Anästhetika beeinflussen diese Regulationsmechanismen, indem sie die Schwelle zum Einsetzen der thermoregulatorischen Vasokonstriktion unter die der tatsächlichen Körperkerntemperatur senken. Dadurch wird in der Einleitung die bisher aktivierte thermoregulatorische Vasokonstriktion aufgehoben, wodurch eine Vasodilatation resultiert, die zu einem Abstrom des kalten Blutes aus der Peripherie in den Körperkern und somit zu einem schnellen Temperaturabfall führt. Das Absenken des Schwellenwertes für thermoregulatorische Vasokonstriktion durch Anästhetika wird für Opiate (Kurz et al. 1995a), Hypnotika (Matsukawa et al. 1995a) und volatile Anästhetika (Ozaki et al. 1995) beschrieben. Des Weiteren führen Anästhetika selber in unterschiedlichem Ausmaß zu einer direkten Vasodilatation. So weist Propofol eine stärkere Vasodilatation als Sevofluran auf, wodurch der Abfall der Körperkerntemperatur größer ist (lkeda et al. 1999).

Die Höhe des Temperaturabfalls nach der Einleitung hängt von der Differenz des Temperaturgradienten zwischen dem Körperkern und der Peripherie ab. Ist die Peripherie stark ausgekühlt, wie z. B. nach längerem Aufenthalt in kalten Umgebungstemperaturen, resultiert ein größerer Abfall der Körperkerntemperatur. So berichten Matsukawa et al. (1995b) in ihrer Initialstudie zu diesem Thema von einem Temperaturabfall von $1,6{ }^{\circ} \mathrm{C}$ innerhalb der ersten Stunde nach Narkoseeinleitung. Werden dagegen Maßnahmen durchgeführt, die den Wärmegehalt der Körperperipherie erhöhen, wie z. B. die Verhinderung von Wärmeverlusten durch eine gute Isolation oder durch Wärmezufuhr mittels aktiver Vorwärmung, resultiert ein geringerer Abfall der Körperkerntemperatur. Daher finden sich in anderen Studien auch geringere Temperaturabfälle von 0,4 bis $1 \mathrm{C}$ nach der Narkoseeinleitung (Brauer et al. 2000, Kurz et al. 1996).

\subsubsection{Lineare Phase}

Die zweite Phase wird als lineare Phase bezeichnet und ist durch einen weiteren, jedoch langsameren Abfall der Körperkerntemperatur während der folgenden zwei bis drei Stunden gekennzeichnet. Dieser resultiert aus einer negativen Wärmebilanz, in der die Wärmeproduktion des Körpers gegenüber den Wärmeverlusten an die Umgebung unzureichend ist. Die Wärmeproduktion eines unbekleideten Körpers beträgt circa ein Watt pro Kilogramm Körpergewicht, wobei dieser Wert während einer Narkose um bis zu $40 \%$ reduziert wird (Sessler 2000). Demgegenüber stehen Wärmeverluste, die bei einer unbekleideten Person im Operationssaal zwischen 180 und $262 \mathrm{~W}$ betragen (Brauer et al. 2006). Dabei wird der größte Teil der Körperwärme mit bis zu circa $90 \%$ über die luftexponierte 
Haut per Luftleitung (Konvektion) und Strahlung (Radiation) abgegeben. Wärmeverluste durch direkte Wärmeleitung (Konduktion) über den Rücken oder durch Verdunstung (Evaporation) über Haut und Atemwege sind gering (Sessler 2000). Insgesamt ist der Wärmeverlust von der Temperaturdifferenz zwischen der Haut und der Umgebungstemperatur im Operationssaal abhängig (Morris 1971). Des Weiteren trägt der Einsatz von kalten Infusionslösungen in Abhängigkeit von den Infusionsgeschwindigkeiten einen weiteren Teil zu der negativen Wärmebilanz bei (Brauer et al. 2006). Der Anteil der Wärmeverluste per Evaporation über das Operationsgebiet ist erst bei langen und großen abdominellen Operationen von klinischer Bedeutung (Lamke et al. 1977).

\subsubsection{Plateauphase}

Die letzte Phase des Temperaturverlaufs während einer Allgemeinanästhesie wird als Plateauphase bezeichnet, in der die Körperkerntemperatur weitestgehend stabilisiert wird. Dieses Temperaturniveau resultiert aus einem ausgeglichenen Verhältnis von Wärmegewinn durch Wärmeproduktion und Wärmeverlusten. In Abhängigkeit von dem Einfluss thermoregulatorischer Mechanismen wird zwischen einem passiven und einem aktiven Plateau unterschieden. Im Gegensatz zum passiven Plateau kann die Körperkerntemperatur beim aktiven Plateau nur mithilfe der wieder aktivierbaren thermoregulatorischen Vasokonstriktion konstant gehalten werden da die Wärmeverluste weiterhin die Wärmeproduktion übersteigen. Durch die thermoregulatorische Vasokonstriktion wird der Blutfluss von der Peripherie zum Körperkern reduziert und darüber wieder eine funktionelle Trennung von Körperkern und Körperschale etabliert (Sessler 2000). In der Folge bleibt die Körperkerntemperatur konstant, während sich die Körperperipherie weiter abkühlt.

\subsection{Physikalische Grundlagen des Wärmetransfers}

\subsubsection{Wärmefluss}

Der Wärmefluss beschreibt als physikalische Größe den Austausch thermischer Energie (Wärme) pro Zeiteinheit zwischen zwei Körpern bzw. zwischen einem Körper und der Umwelt bei unterschiedlichen Temperaturen (The Commission for Thermal Physiology of the International Union of Physiological Sciences 2001). Der Wärmefluss berechnet sich als zeitliche Änderung der Wärmemenge bezogen auf die Körperoberfläche (Wärmemenge $Q$ pro Zeit t und Fläche $A$ ) und hat die SI-Einheit $\mathrm{W}^{*} \mathrm{~m}^{-2}$. 


\subsubsection{Wärmeaustausch}

Der Wärmeaustausch erfolgt als Wärmefluss zwischen zwei Körpern bzw. zwischen einem Körper und der Umwelt. Dabei erfolgt der Wärmeaustausch immer vom Ort höherer Temperatur zum Ort niedrigerer Temperatur.

Physikalisch betrachtet gilt für den Wärmeaustausch $(\dot{Q})$ folgende Abhängigkeit:

$\dot{Q}=\Delta \mathrm{T} * \mathrm{~A} * \mathrm{~h} \quad($ English 2001)

$\Delta \mathrm{T}=$ Temperaturgradient $\left({ }^{\circ} \mathrm{C}\right)$

$A=$ Fläche $\left(m^{2}\right)$

$\mathrm{h}=$ Wärmeaustauschkoeffizient $\left(\mathrm{W}^{*} \mathrm{~m}^{-2 *{ }^{\circ}} \mathrm{C}^{-1}\right)$

Somit ist der Wärmeaustausch proportional abhängig von:

1. dem Energiegradienten zwischen zwei Oberflächen.

a) Der Energiegradient ist bei Konduktion, Konvektion und Radiation als treibende Kraft die Temperaturdifferenz.

b) Der Energiegradient ist bei der Evaporation als treibende Kraft die Differenz im Wasserstoffpartialdruck.

2. der Größe der Oberfläche, über die der Wärmeaustausch erfolgt.

3. dem Koeffizienten, der die Effektivität des Wärmeaustausches bestimmt.

\subsubsection{Prinzipien der Wärmeübertragung}

Der Wärmeaustausch erfolgt über vier verschiedene physikalische Prinzipien: Konduktion, Konvektion, Radiation und Evaporation.

\subsubsection{Konduktion}

Mittels Konduktion erfolgt die Wärmeübertragung zwischen zwei Molekülen bei direktem Kontakt. Die Temperaturübertragung folgt dem Gradienten von dem Ort höherer zu dem Ort niedrigerer Energie. Dabei verändern die Moleküle ihren Standort aufgrund der hohen intermolekularen Bindung in Festkörpern nicht. Daher kann per Konduktion Wärme nur bei direktem Kontakt in oder zwischen zwei Festkörpern übertragen werden (English 2001). 


\subsubsection{Konvektion}

Bei Konvektion erfolgt die Wärmeübertragung zwischen Molekülen von Flüssigkeiten oder Gasen, wobei diese aufgrund ihrer schwächeren intermolekularen Bindung ihren Standort verändern. Die Energieübertragung erfolgt entlang des Temperaturgradienten vom Ort höherer zum Ort niedrigerer Temperaturen. Ist dieser die alleinige treibende Kraft des Wärmeaustausches, wird der Vorgang freie Konvektion genannt. Beeinflusst neben dem Temperaturgradienten eine weitere äußere Kraft die Molekülbewegung, wie z.B. ein laminarer Flow, so bezeichnet man den Vorgang als erzwungene Konvektion (English 2001).

\subsubsection{Radiation}

Bei Radiation erfolgt die Wärmeübertragung mittels elektromagnetischer Strahlung zwischen zwei Körpern. Sie folgt dem Temperaturgradienten, ohne dass ein externes Medium zur Wärmeübertragung benötigt wird. Trifft die Strahlung auf einen Körper, wird sie in Abhängigkeit von seinen Materialeigenschaften entweder absorbiert, reflektiert oder durchdringt diesen. Der menschliche Körper absorbiert auftreffende Infrarotstrahlung fast vollständig, ein geringer Teil wird reflektiert. Insgesamt ist vor allem die langwellige Infrarotstrahlung für die Wärmeübertragung beim Menschen verantwortlich (English 2001).

\subsubsection{Evaporation}

Bei der Evaporation erfolgt die Wärmeabgabe durch die Verdunstung von Feuchtigkeit. Im Gegensatz zu den vorangegangen Prinzipien ist nicht der Temperaturgradient sondern die Differenz des Wasserdampfpartialdrucks die treibende Kraft für den Wärmeaustausch. An einem Ort mit hoher Feuchtigkeit besteht ein hoher Wasserdampfpartialdruck. Die Wassermoleküle diffundieren nun von dem Ort mit hohem Wasserdampfpartialdruck an den Ort mit niedrigem Wasserdampfpartialdruck. Die Wärmeabgabe durch Evaporation wird durch die Höhe des Wasserdampfpartialdruckgradienten bestimmt. Des Weiteren verstärkt eine Zunahme der Luftgeschwindigkeit das Ausmaß der evaporativen Wärmeabgabe linear (English 2001). 


\subsection{Prävention perioperativer Hypothermie}

\subsubsection{Prewarming}

Die aktive Vorwärmung der Haut und Körperperipherie des Patienten vor Narkoseeinleitung wird als Prewarming bezeichnet (Hooper 2009). Durch Vorwärmung wird der typische Abfall der Körperkerntemperatur in der ersten Stunde nach der Narkoseeinleitung reduziert (Hooper 2009). Die durch Anästhetika induzierte Vasodilatation führt zu einer Umverteilung des Blutes aus dem warmen Körperkern in die kalte Körperperipherie. Wie Matsukawa et al. (1995b) an Probanden zeigen, verursacht die Blutumverteilung zu 81 \% den schnellen Abfall der Körperkerntemperatur nach Narkoseeinleitung. Prewarming trägt über zwei Mechanismen dazu bei diesen Temperaturabfall zu reduzieren. Durch Prewarming wird die Temperatur in der Körperperipherie erhöht sowie eine periphere Vasodilatation ausgelöst. Insgesamt resultiert dadurch eine Reduktion des Temperaturgradienten zwischen der Körperperipherie und dem Körperkern. Dieser Vorgang reduziert den Abfall der Körperkerntemperatur und damit das Auftreten von Hypothermie wie viele Studien zeigen (de Brito Poveda et al. 2013, Hynson et al. 1993). Hynson et al. (1993) demonstrieren dies in einer Studie an Probanden, die nach zwei Stunden Prewarming eine Allgemeinanästhesie ohne Operation erhielten. Bei vergleichbaren Ausgangstemperaturen fällt die Temperatur nach Narkoseeinleitung ohne Vorwärmung auf $34,9^{\circ} \mathrm{C}$ verglichen mit $36,1^{\circ} \mathrm{C}$ nach Vorwärmung ab. In einer weiteren Studie an Probanden wird die optimale Dauer der Vorwärmung untersucht. Dabei zeigen Sessler et al. (1995), dass durch eine Vorwärmdauer von 30 bis 60 Minuten über konvektive Luftwärmer eine ausreichende Wärmeenergie übertragen wird Dabei trat innerhalb der ersten Stunde kein Diskomfort bei den Probanden auf, wohingegen die Hälfte der Probanden mit vermehrtem Schwitzen und Überwärmung nach zwei Stunden Wärmezufuhr reagierte. Eine aktuelle Metaanalyse hat 14 Studien eingeschlossen, welche die Effektivität von Vorwärmung untersuchen. In zwölf Studien wird ein signifikant reduzierter Abfall der Körperkerntemperatur nach Prewarming durch konvektive Luftwärmer gezeigt. Dabei wird in elf Studien eine Vorwärmdauer von 30 bis 60 Minuten angewandt (de Brito Proveda et al. 2013). Aktuelle Untersuchungen von Horn et al. (2012) zeigen, dass bereits ein aktives Prewarming von zehn Minuten ausreicht um das Auftreten von Hypothermie unter Allgemeinanästhesie signifikant zu reduzieren. Von den nicht vorgewärmten Patienten wurden 69 \% hypotherm, hingegen nur $13 \%$ der Patienten die eine zehnminütige Vorwärmung erhielten. Eine Verlängerung der Vorwärmdauer auf 20 oder 30 Minuten reduziert das Auftreten von Hypothermie nicht mehr signifikant auf $7 \%$ und $6 \%$. 
Schon alleiniges Prewarming über konvektive Luftwärmer reduziert gegenüber einer Isolation mit Baumwolllaken die Rate intraoperativer Hypothermie signifikant bei laparoskopischen (Camus et al. 1995), allgemeinchirurgischen (Melling et al. 2001), orthopädischen (Fossum et al. 2001) und Off-Pump-kardiochirurgischen Operationen (Kim et al. 2006). Der prä- und intraoperative Einsatz von konvektiven Luftwärmern führt zu einer Reduktion intraoperativer Hypothermie gegenüber der Isolation mit einem Baumwolllaken bei allgemeinchirurgischen Eingriffen (Vanni et al. 2003) und elektiven Kaiserschnitten (Horn et al. 2002). Ein signifikant geringerer Abfall der Körperkerntemperatur wird ebenfalls bei Wirbelsäuleneingriffen (Andrzejowski et al. 2008) und Hüftoperationen (Just et al. 1993) gezeigt, wenn die Kontrollgruppe statt dem Baumwolllaken intraoperativ Wärmezufuhr über konvektive Luftwärmer erhalten hat. In den Untersuchungen von Andrzejowski et al. (2008) führt Prewarming zu einem um 0,3 ${ }^{\circ} \mathrm{C}$ geringeren Abfall der Körperkerntemperatur, wodurch ein statistisch signifikanter Unterschied im Einhalten von Normothermie in der Vorwärmgruppe mit 68 \% verglichen mit $43 \%$ in der Kontrollgruppe erreicht wird.

\subsubsection{Intraoperative Wärmeprotektionsverfahren}

\subsubsection{Wärmeprotektion durch Erhöhung der Raumtemperatur}

Die Erhöhung der Raumtemperatur stellt ein passives Verfahren der Wärmeprotektion dar. Hierbei verringern sich die Wärmeverluste über die Haut um $10 \%$ pro $1^{\circ} \mathrm{C}$ erhöhter Raumtemperatur (Brauer et al. 2006). In einer frühen Studie findet sich bei Raumtemperaturen von 21 bis $24^{\circ} \mathrm{C}$ ein Anteil von $30 \%$ hypothermer Patienten, wohingegen unter $21^{\circ} \mathrm{C}$ alle Patienten hypotherm wurden und bei Raumtemperaturen von 24 bis $26^{\circ} \mathrm{C}$ alle Patienten normotherm blieben (Morris 1971). Auch neuere Studien zeigen, dass sich der Abfall der Körperkerntemperatur während einer Allgemeinanästhesie in einem warmen Operationssaal mit $24{ }^{\circ} \mathrm{C}$, verglichen mit einer Raumtemperatur von $21{ }^{\circ} \mathrm{C}$, reduziert (Frank et al. 1992). Um allerdings bei Erwachsenen eine ausgeglichene Wärmebilanz allein durch erhöhte Umgebungstemperaturen zu erreichen, sind Temperaturen im Operationssaal von mehr als $26^{\circ} \mathrm{C}$ nötig (El-Gamal et al. 2000).

\subsubsection{Wärmeprotektion durch Isolation}

Durch eine Isolation des Körpers wird der Wärmeverlust um circa 30 \% reduziert. Hierbei bewirkt die Abdeckung eine Reduktion des Flusses warmer Luft vom Körper an die Umgebung und reduziert damit die konvektiven und radiativen Wärmeverluste (Sessler et al. 1991a). Dabei ist die Effektivität der Isolation proportional von der Größe der bedeckten Körperoberfläche sowie vom Isolationswert 
des Materials abhängig. Bei einer Studie an Probanden zeigen sich bei einer begrenzten Anzahl unterschiedlicher kommerzieller Isolationsmaterialien kaum Unterschiede in der Reduktion der Wärmeverluste (Sessler et al. 1991a). Dagegen dokumentieren Brauer et al. (2007) bei dem Vergleich verschiedener effektiver, jedoch im Operationssaal zum Teil unüblicher Isolationsmaterialien große Unterschiede im Isolationswert. Diese variieren von 0,01 clo bei einer Plastikfolie bis zu 2,79 clo bei einer zweilagigen Krankenhaussteppdecke. Dabei kann durch den Einsatz mehrlagiger Bedeckung zwar der Isolationswert erhöht werden, allerdings ohne den Wärmeverlust stark zu reduzieren. Dies liegt zum einen daran, dass der Haupteffekt der passiven Isolation durch die Reduktion der Luft- und Wärmezirkulation bedingt ist (Sessler und Schroeder 1993). Zum anderen wird der Wärmeverlust durch geringe Isolation zunächst zwar prozentual stark reduziert, wird allerdings eine bereits gut isolierte Oberfläche mit einem besseren Isolationsmaterial bedeckt, resultiert dagegen prozentual nur eine geringere Reduktion des Wärmeflusses (Brauer et al. 2004c).

\subsubsection{Wärmeprotektion durch Heizmatten unter dem Rücken}

Wärmeprotektion durch eine unter dem Rücken liegende, Wasser-zirkulierende Matte vermittelt den Wärmetransfer mittels Konduktion. Die konduktiven Wärmeverluste über den Rücken sind mit 3 bis 5 Watt gering, da 90 \% der Körperwärme über die vordere Körperfläche verloren gehen (Hynson und Sessler 1992). Daher kann die Wärmebilanz durch über den Rücken zugeführte Wärmemaßnahmen auch nur gering beeinflusst werden. So zeigt sich der Einsatz konduktiver, unter den Rücken gelegter Wärmematten gegenüber konvektiven Luftwärmern in der Verhinderung perioperativer Hypothermie unterlegen (Bock et al. 1998, Hynson und Sessler 1992, Hynson et al. 1993). Da der Wärmefluss von der Wärmematte zum Körper auch von der Temperaturdifferenz abhängig ist, stellt diese einen weiteren limitierenden Faktor der Wärmeübertragung dar. English et al. (1990) zeigen, dass bei normothermen Probanden die Temperaturdifferenz nur $0,7^{\circ} \mathrm{C}$ bei einer Wassermattentemperatur von $40^{\circ} \mathrm{C}$ beträgt. Des Weiteren beeinflusst die Kontaktfläche zwischen der Wärmematte und dem Rücken die Größe der Wärmeübertragung und kann z. B. durch eine anpassungsfähigere Geloberfläche bei wärmenden Wassermatten verbessert werden (Brauer et al. 2004a). Allerdings bewirkt diese Gelschicht als zusätzliche Isolation einen schlechteren Wärmetransport von der Wassermatte auf die Körperfläche, sodass es bei einer Erhöhung der Wassertemperatur um $1{ }^{\circ} \mathrm{C}$ nur zu einer Erhöhung der Mattentemperatur um $0,5^{\circ} \mathrm{C}$ kommt (Brauer et al. 2004a). Dagegen werden druckbedingte 
Verbrennungen durch Wärmematten bereits bei einer Temperatur von $38^{\circ} \mathrm{C}$ beschrieben (Crino und Nagel 1968).

\subsubsection{Wärmeprotektion durch auf den Körper gelegte Heizmatten}

Da ein Großteil der Wärmeverluste über die Körperoberfläche entsteht, ist die Wärmeprotektion durch auf den Körper gelegte Heizmatten viel effektiver als durch unter dem Rücken platzierte. Dabei ist die Effektivität von der Größe der Kontaktfläche zwischen Körper und Decke abhängig. Hierbei erfolgt der Wärmetransfer mittels Konduktion, wohingegen ohne direkten Kontakt dieser nur per Radiation erfolgt. Eine Studie an Probanden zeigt, dass durch den großflächigen Einsatz einer auf den Körper gelegten Wassermatte die Wärmeverluste an die Umwelt effektiv reduziert werden können. Die Reduktion der Wärmeverluste ist hier vergleichbar mit dem Einsatz des konvektiven Luftwärmers BairHugger® auf mittlerer Wärmestufe (Sessler und Moayeri 1990). In weiteren Studien erweisen sich die Wärmematten, die entweder mit Wasser (Brauer et al. 2004a, Grocott et al. 2004, Janicki et al. 2001) oder auch elektrisch (Matsuzaki et al. 2003) betrieben sind, als ausreichend effektiv in der Vermeidung perioperativer Hypothermie. Studien an konduktiven Wassermattenanzügen führen verglichen mit konvektiver Luftwärmung zu einer höheren Körperkerntemperatur (Janicki et al. 2001, Janicki et al. 2002).

\subsubsection{Wärmeprotektion durch konvektive Luftwärmer}

Wärmeprotektion mittels konvektiver Luftwärmer ist sehr effektiv, da die Wärmeverluste des Körpers per Radiation und Konvektion stark reduziert werden und aktiv Wärme zugeführt wird (Brauer et al. 2009). Das Wärmesystem besteht aus einem elektrischen Wärmelüfter, welcher über einen oder mehrere Gebläseschläuche gewärmte Luft in eine Wärmedecke befördert. Die gewärmte Luft verteilt sich in der Decke und verlässt diese wieder an der Unterseite. Durch die Wärmeübertragung mittels Konvektion ist im Vergleich zu konduktiven Wärmesystemen kein enger Kontakt zwischen Körper und Decke erforderlich. Die Wärmedecke wird in verschiedenen Materialien für die einmalige oder wiederverwendbare Anwendung sowie in unterschiedlichen Größen und Ausführungen angeboten. Neben den gängigen Varianten der Oberkörper-, Unterkörper- oder Ganzkörperunterlegdecke existieren noch viele weitere Deckenmodelle wie z. B. in Form eines Wärmehemdes (Andrzejowski et al. 2008). Die Effektivität des Wärmeaustausches zwischen der Decke und der Körperoberfläche ist sowohl abhängig vom erzeugten Wärmefluss des Wärmelüfters als auch vom Wärmeaustauschkoeffizienten der Wärmedecke sowie dem mittleren Temperatur- 
gradienten zwischen der Haut und der Decke. Des Weiteren werden die Wärmeverluste durch Radiation und Konvektion umso besser reduziert, je größer die bedeckte Körperoberfläche ist (Brauer et al. 2003). So finden Brauer et al. (2003) bei Untersuchungen am Modell bei Unterkörperwärme-decken zwar einen geringeren Wärmefluss als bei Oberkörperwärmedecken (Brauer et al. 2002), dennoch reduzieren Unterkörperwärmedecken die Wärmeverluste aufgrund der gröBeren Oberflächenabdeckung besser als Oberkörperwärmedecken. Es resultiert eine positive Wärmebilanz von 78 bis $88 \mathrm{~W}$ bei der Unterkörperwärmedecke im Vergleich zu 40 bis $64 \mathrm{~W}$ bei einer Oberkörperwärmedecke. In vielen Studien zeigt sich der Einsatz konvektiver Luftwärmer bei verschiedenen Operationen als effektive Maßnahme zur Reduktion perioperativer Hypothermie (Berti et al. 1997, Bock et al. 1998, Hynson und Sessler 1992, Ng et al. 2003). Dennoch kann der alleinige Einsatz konvektiver Luftwärmer nicht immer Normothermie sicherstellen (Smith et al. 1998). In einer aktuellen Metaanalyse zur Effizienz verschiedener externer Wärmesysteme zeigen sich intraoperative Wärmemaßnahmen durch konvektive Luftwärmer der Isolation durch Baumwolltücher als auch gegenüber radiativer Wärmezufuhr überlegen. So finden sich bei Einsatz konvektiver Luftwärmer um $0,29^{\circ} \mathrm{C}$ bzw. $0,16{ }^{\circ} \mathrm{C}$ höhere Körperkerntemperaturen verglichen mit der Isolation durch Baumwolltücher bzw. durch radiative Wärmemaßnahmen. Allerdings führt der Einsatz von konduktiven Wassermattenanzügen gegenüber konvektiven Luftwärmern zu einer mittleren höheren Körperkerntemperatur von $0,73^{\circ} \mathrm{C}$ (Galvao et al. 2010).

\subsubsection{Sonstige Verfahren}

Es existieren eine Anzahl weiterer externer und interner Wärmeprotektionsverfahren, deren Effektivität jedoch meist eingeschränkt ist.

Mittels Atemgasklimatisierung ist keine relevante Wärmezufuhr möglich, da die Wärmeverluste über den Respirationstrakt nur $10 \%$ der produzierten Körperwärme betragen (Sessler 2001). Durch den Einsatz von aktiver (Hynson und Sessler 1992) oder passiver (Bickler und Sessler 1990) Atemgasklimatisierung werden die Feuchtigkeitsverluste durch Verdunstung reduziert, sodass eine positive Wärmebilanz von 6 bis 8 W resultiert (Brauer et al. 2006).

Ein weiteres externes Verfahren zur Wärmeprotektion sind Infrarotstrahler, wobei die Wärmeenergie über Photonen auf den Körper übertragen wird, ohne dass ein direkter Kontakt nötig ist. Die Menge der übertragenen Energie durch Infrarotstrahler steigt mit der Nähe zum Körper und der Größe der bestrahlten Fläche. Allerdings sind die frei zugänglichen Körperflächen im intraoperativen Bereich 
häufig begrenzt, sodass je nach operativem Eingriff keine ausreichende Wärmeenergie zum Wärmeerhalt übertragen werden kann. So zeigen sich Infrarotstrahler in der Vermeidung intraoperativer Hypothermie sowohl gleichwertig (Wong et al. 2004) als auch den konvektiven Luftwärmern unterlegen (Lee et al. 2004). Ein Nachteil besteht in dem ungehinderten großen Wärmeverlust per Konvektion, welcher ungefähr die Hälfte der Wärmeverluste über die Haut ausmacht (Sessler 2001) sowie der großen Wärmebelastung des in der Nähe arbeitenden Personals. Daher bietet sich der Einsatz von Infrarotstrahlern eher im prä- oder postoperativen Bereich an und wird z. B. als fundiertes Verfahren des Wärmeerhalts in der intensivmedizinischen Versorgung von Neonaten eingesetzt (Sessler 2001).

Zu den möglichen internen Wärmeprotektionsverfahren zählen der Einsatz von Ösophaguswärmern, Infusionswärmern und die Zufuhr von Aminosäuren. Ösophaguswärmer zeigen sich wenig effektiv, um einen ausreichenden konduktiven Wärmetransfer zur Prävention intraoperativer Hypothermie zu ermöglichen (Rasmussen et al. 1998). Dies liegt daran, dass sowohl nur eine geringe Austauschfläche als auch nur ein geringer Temperaturgradient zur Ösophagusschleimhaut zum Wärmeaustausch zur Verfügung steht. Ein höherer Temperaturgradient zwischen Ösophaguswärmer und Ösophagusschleimhaut ist aufgrund der Gefahr von Verbrennungen nicht realisierbar (Brauer und Weyland 1998).

Die Applikation von kalten Infusionen oder Blutprodukten führt pro appliziertem Liter zu einem Abfall der Körperkerntemperatur von 0,25 ${ }^{\circ} \mathrm{C}$ (Sessler 1997). Mit dem Einsatz von Infusionswärmern können die Wärmeverluste, die durch die Zufuhr großer Mengen ungewärmter Flüssigkeiten entstehen, effektiv reduziert werden (Sessler 2001). Dabei zeigen sich verschiedene kommerzielle Infusionswärmesysteme bei unterschiedlichen Flussraten suffizient (Schmidt et al. 1996). Allerdings kann durch den Einsatz von Infusionswärmern keine relevante Wärmemenge zugeführt werden. Daher ersetzen sie als alleinige Maßnahme nicht den Einsatz weiterer isolierender oder aktiv wärmender Maßnahmen zur Vermeidung perioperativer Hypothermie (Sessler 2001).

Durch die präoperative Zufuhr von Aminosäuren wird der Stoffwechsel des Körpers gesteigert und dabei die Wärmeproduktion erhöht. Dies bewirkt eine Erhöhung der Körperkerntemperatur sowie der Thermoregulationsschwelle (Sellden et al. 1996). In einer Studie an Patienten führt die präoperative Zufuhr von Aminosäuren zu einem geringeren Abfall der Körperkerntemperatur von $0,6{ }^{\circ} \mathrm{C}$ sowie einem reduzierten Auftreten von Shivering (Sellden und Lindahl 1998). 


\subsection{Risiken der Wärmetherapie}

\subsubsection{Auftreten von Verbrennungen}

Aktive Wärmesysteme unterliegen festgelegten Sicherheitsnormen (International Electrotechnical Commission 2009), wodurch das Risiko für Verbrennungen weitestgehend minimiert wird. Dennoch bleibt beim Einsatz von aktiven Wärmemaßnahmen prinzipiell ein Risiko für Verbrennungen bestehen und wird in einzelnen Fallberichten auch dokumentiert. So finden sich in der Literatur Berichte von Verbrennungen beim Einsatz von Heizmatten (Crino und Nagel 1968, Gali et al. 2003) oder auch konvektiven Luftwärmern (Azzam und Krock 1995, Truell et al. 2000). Neben Gerätefehlfunktionen kann auch der unsachgemäße Gebrauch zu Verbrennungen führen. Dazu zählt zum Beispiel die Kombination von Decke und Gebläsegerät verschiedener Hersteller oder das Hosing, also der Einsatz konvektiver Luftwärmer ohne spezielle Wärmematte (Brauer et al. 2006). Des Weiteren stellt die Kombination aus hohen Temperaturen und erhöhtem Druck ein besonderes Risiko dar, da hier die Durchblutung reduziert wird (Sessler 2001). Dadurch kann das Auftreten von Verbrennungen, z. B. bei dem Einsatz eines unter dem Rücken liegenden konduktiven Wärmesystems, bei empfindlichen Patienten bereits bei niedrigeren Temperaturen begünstigt sein (Crino und Nagel 1968).

\subsubsection{Störung des Luftstroms in Laminar-Airflow-Sälen}

Beim Einsatz von konvektiven Luftwärmern in Laminar-Airflow-Sälen bestehen die theoretischen Bedenken, dass es durch eine Veränderung des Luftstroms zu erhöhten Keimzahlen im Operationsgebiet und vermehrten Wundinfektionen kommt. Studien an Probanden zeigen dagegen, dass beim Einsatz von konvektiven Luftwärmern keine erhöhte Keimzahl im Operationsgebiet nachweisbar ist (Sharp et al. 2002, Zink und laizzo 1993). Durch Filter am Wärmebläser wird die Kontamination bei bodennaher Luftansaugung und Umverteilung über die Wärmedecke in das Operationsgebiet verhindert (Sharp et al. 2002). Auch bei Patienten zeigt sich beim Einsatz des konvektiven Wärmesystems BairHugger ${ }^{\circledR}$ keine erhöhte Infektionsrate sondern sogar eine reduzierte Keimzahl in der Umgebung des Operationsgebietes (Huang et al. 2003). 


\subsubsection{Nutzen-Risiko-Relation der Prävention von Hypothermie}

Mögliche Risiken und Nachteile des perioperativen Temperaturmanagements, wie z. B. körperliche Schäden durch Verbrennungen oder wirtschaftliche Aufwendungen für Wärmemaßnahmen und Temperaturüberwachung, werden durch den Nutzen eines adäquaten Temperaturerhalts deutlich überwogen. Wie eine Metaanalyse darstellt (Mahoney und Odom 1999), erhöhen sich die Kosten um 2500 bis 7000 für jeden hypothermen Patienten, dessen Körperkerntemperatur durchschnittlich um $1,5{ }^{\circ} \mathrm{C}$ von der eines normothermen Patienten abweicht. Diese Kosten entstehen durch eine erhöhte Rate an Komplikationen und Morbiditäten, die zusätzliche Therapien und einen verlängerten Krankenhaus- oder Intensivaufenthalt notwendig machen. Insgesamt resultiert ein eindeutiges NutzenRisiko-Verhältnis sowohl aus ökonomischer als auch ethischer Sicht für effektive Maßnahmen zur Prävention perioperativer Hypothermie (Mahoney und Odom 1999). Daher wird in den Leitlinien des National Institute for Clinical Excellence (2008) empfohlen, neben der Erwärmung der zu infundierenden Flüssigkeiten auf $37^{\circ} \mathrm{C}$, jeden Patienten, der eine Operationsdauer von über 30 Minuten erwartet, nach der Einleitung mit aktiver konvektiver Wärmezufuhr zu versorgen. Bei Patienten, die ein höheres Risiko für die Entwicklung perioperativer Hypothermie aufweisen, sollen die Wärmemaßnahmen unabhängig von der Operationsdauer erfolgen. Des Weiteren soll eine aktive Wärmtherapie prä-, intra- und postoperativ immer angewendet werden, wenn die Körperkerntemperatur unter $36^{\circ} \mathrm{C}$ fällt (NICE 2008).

\subsection{Inzidenz perioperativer Hypothermie bei HNO-Patienten}

\subsubsection{Prävention und Risiken bei HNO-chirurgischen Eingriffen}

Das Risiko für die Entwicklung einer perioperativen Hypothermie bei HNOchirurgischen Patienten wird im Vergleich zu größeren chirurgischen Eingriffen häufig als gering eingeschätzt. Ursächlich dafür scheint, dass Patienten bedingt durch das kleinere Operationsgebiet eine geringe Wundfläche aufweisen, keine großen Volumenverluste ausgeglichen werden müssen und prinzipiell eine große Fläche zur passiven Isolation zur Verfügung steht (Perl et al. 2012). Die in der Literatur existierenden Studien zur Inzidenz (Abelha et al. 2005, Karalapillai et al. 2009) und Prävention perioperativer Hypothermie (de Brito Poveda et al. 2013) sind zumeist an Patienten mit großen unfallchirurgischen oder allgemeinchirurgischen Operationen durchgeführt worden. Dahingegen ist die Studienlage bezüglich der Inzidenz von Hypothermie und dem Auftreten der durch die Hypothermie bedingten Komplikationen bei HNO-chirurgischen Patienten übersichtlich. In 
zwei großen retrospektiven Untersuchungen an über 5.000 bzw. über 50.000 Patienten werden Inzidenzen für Hypothermie nach größeren HNO-chirurgischen sowie plastischen Eingriffen von 31 \% (Karalapillai et al. 2009) und 44,6 \% (Karalapillai et al. 2013) angegeben. Bemerkenswerterweise liegen diese Inzidenzen in der jeweiligen Studie in einer vergleichbaren Größenordnung wie nach allgemeinchirurgischen oder unfallchirurgischen Operationen. Studien, die an Patienten durchgeführt wurden, die sich kleinen bis mittleren HNO-chirurgischen Eingriffen unterzogen haben, zeigen Inzidenzen für perioperative Hypothermie von 60 \% (Perl et al. 2012) und $67 \%$ (Agrawal et al. 2003) in der ungewärmten Kontrollgruppe. Betrachtet man die Inzidenzen für schwere Hypothermie mit Körperkerntemperaturen unter $35^{\circ} \mathrm{C}$, so wird diese in zwei älteren Studien bei mittleren HNO-chirurgischen Eingriffen mit 10 \% (Agrawal et al. 2003) und bei groBen HNO-Operationen wie tumorchirurgischen Lappenplastiken mit $11 \%$ (Sumer et al. 2009) angegeben.

Aber auch die Rate an Komplikationen wird durch das Auftreten von Hypothermie beeinflusst (Agrawal et al. 2003). So zeigen Sumer et al. (2009), dass Patienten mit schwerer Hypothermie nach tumorchirurgischen Lappenplastiken ein fünffach erhöhtes Risiko für das Auftreten von Komplikationen aufweisen, zu denen z. B. Lappenverlust, Hämatombildung und weitere zählen. Dabei wirkt sich zudem auch der Schweregrad der Hypothermie auf die Rate postoperativer Komplikationen aus. So ist bei mittleren HNO-chirurgischen Eingriffen der Abfall der Körperkerntemperatur unter $35^{\circ} \mathrm{C}$ in $71 \%$ mit Komplikationen assoziiert, verglichen mit einer Komplikationsrate von $7 \%$, wenn nur eine milde Hypothermie auftritt (Agrawal et al. 2003).

Zusammenfassend kann aus den vorliegenden Studien zur Inzidenz und Prävention von Hypothermie bei HNO-chirurgischen Patienten gezeigt werden, dass diese ebenfalls in relevantem Maße auftritt und mit einer bedeutenden Anzahl an Komplikationen verbunden ist.

\subsection{Das neue konduktive Wärmesystem Barrier ${ }^{\circledR}$ Easywarm ${ }^{\circledR}$}

\subsubsection{Möglichkeiten des perioperativen Wärmemanagements}

Um die Patienten in der gesamten perioperativen Phase mit einem adäquaten Wärmemanagement versorgen zu können, müssen bestimmte Voraussetzungen erfüllt werden. Im Idealfall erhält der Patient präoperativ aktive Wärmemaßnahmen in Form von Prewarming (de Brito Poveda et al. 2013), welches bereits auf der Station oder in der Wartezone durchgeführt wird. Hier bietet die Wärmedecke 
Barrier ${ }^{\circledR}$ Easywarm ${ }^{\circledR}$ mehrere Vorteile, da sie einfach anzuwenden ist und von dem Patienten selber aktiviert werden kann, sowie als Einmalartikel an jedem Ort flexibel einsetzbar ist. Des Weiteren ermöglicht der stromlose Betrieb von Barrier ${ }^{\circledR}$ Easywarm ${ }^{\circledR}$ eine ununterbrochene Wärmezufuhr von der Station, über die Wartezone, intraoperativ sowie postoperativ im Aufwachraum.

Daher bietet das neue konduktive Wärmesystem Barrier® Easywarm ${ }^{\circledR}$ viele theoretische Vorteile und wird in dieser Studie auf seine Effizienz in der Vermeidung perioperativer Hypothermie untersucht.

\subsection{Fragestellung}

Die vorliegende Arbeit hat zum Ziel, folgende Fragestellungen zu beantworten:

1. Sind HNO-Patienten von einem relevanten Risiko für perioperative Hypothermie betroffen?

2. Wird durch den Einsatz des neuen konduktiven Wärmesystems Barrier® Easywarm ${ }^{\circledR}$ das Auftreten perioperativer Hypothermie signifikant reduziert? 


\section{MATERIAL und METHODEN}

\subsection{Randomisierung und Patientenrekrutierung}

Das Studienprotokoll der prospektiv randomisierten kontrollierten Studie wurde unter der Antragsnummer 10/7/12 durch die zuständige örtliche Ethikkommission am 19.07.2012 positiv beschieden und beim Deutschen Register klinischer Studien unter der Nummer DRKS00004523 registriert. Daraufhin erfolgte das Screening der Patienten, die sich laut Operationsplan einem ein- bis dreistündigen operativen Eingriff in der Hals-Nasen-Ohrenheilkunde unterziehen mussten, sowie im Alter zwischen 18 und 75 Jahren waren. Dabei konnten 118 Patienten auf weitere Ein- und Ausschlusskriterien untersucht werden.

Folgende Einschlusskriterien wurden für die Studienteilnehmer festgelegt:

- Geplante Operationsdauer 60 bis 180 Minuten

- Alter 18 bis 75 Jahre

- BMl 20 bis $30 \mathrm{~kg} / \mathrm{m}^{2}$

- Risikogruppe ASA I bis III

- Geplante Narkose mit Remifentanil, Propofol, Rocuronium

Folgende Ausschlusskriterien wurden für die Studienteilnehmer festgelegt:

- Operationszeit unter 60 Minuten sowie über 180 Minuten

- Bekannte Unverträglichkeit gegen Remifentanil, Propofol, Rocuronium

- Schwangerschaft, Stillzeit

- Fieberhafter Infekt

- Klinisch manifeste Hypo- oder Hyperthyreose

Nach Abschluss des Screenings und ausführlicher Aufklärung durch den Prüfarzt willigten 80 Patienten schriftlich in die Studienteilnahme, Datenerhebung und Datenspeicherung ein (siehe Anhang). Diese Patienten erhielten daraufhin einen verschlossenen und blickdichten Umschlag der einen Zettel mit der Einteilung entweder in die Interventions- oder Kontrollgruppe enthielt. Die Briefumschläge waren numerisch von 1 bis 80 durchnummeriert und der Inhalt der Studiengruppenzuteilung war dem aufklärenden Arzt nicht bekannt. Die Aushändigung des Briefumschlags erfolgte analog der Einwilligung. Damit erhielt der erste Patient den Umschlag Nummer 1, der zweite die Nummer 2 und so weiter. Die Abfolge 
der Studiengruppeneinteilung wurde mit einem elektronischen Randomisierungsprogramm (http://www.randomization.com) erstellt, mit dessen Hilfe die Patienten zu gleichen Teilen in die Interventions- und Kontrollgruppe randomisiert wurden.

Die Patienten der Kontrollgruppe wurden mit einer konventionellen Krankenhaussteppdecke bedeckt zur Operationseinschleusung gefahren. Nach dem Umlagern bekamen die Patienten eine frische Bettdecke, mit der sie in den Operationssaal gebracht wurden. Die konventionelle Krankenhaussteppdecke ist mit Trevira (100\% Polyester) gefüllt (Brinkhaus GmbH \& Co. KG, Warendorf, Deutschland) und erzielt einen Isolationswert von 1,29 clo.

Die Patienten der Interventionsgruppe bekamen präoperativ mindestens $30 \mathrm{Mi}-$ nuten vor Narkoseeinleitung die neue konduktive Wärmedecke Barrier® Easywarm ${ }^{\circledR}$ (Barrier ${ }^{\circledR}$ Easywarm $\AA$, Mölnlycke, Erkrath, Deutschland) anstatt der Krankenhaussteppdecke aufgelegt. Die Prewarming Zeit, definiert als Zeit zwischen Deckenauflage und Narkoseeinleitung, wurde dokumentiert. Die Wärmedecke behielten sie während der gesamten perioperativen Phase. Zusätzlich wurden die Füße der Patienten mit einem Stecklaken bedeckt, falls diese frei lagen. Im Aufwachraum wurde auf Verlangen der Patienten die Wärmedecke gegen eine konventionelle Krankenhaussteppdecke ausgetauscht.

\subsection{Die Wärmedecke Barrier ${ }^{\circledR}$ Easywarm ${ }^{\circledR}$}

Die Wärmedecke Barrier ${ }^{\circledR}$ Easywarm $\AA$ hat eine Größe von $152 \mathrm{~cm}$ x $92 \mathrm{~cm}$ und besteht aus mehreren Polypropylen Vlieslagen (Abbildung 1). Sie besitzt zwölf Taschen der Größe $13 \mathrm{~cm} \times 10 \mathrm{~cm}$ an der Oberseite, die mit jeweils einem Wärmepad bestückt sind. Diese sind mit einer Mischung aus Eisen(II)Pulver, Aktivkohle, Natriumchlorid, Wasser und Mineralien gefüllt. Die Lieferung der Decke erfolgt vakuumverpackt und damit in einem inaktiven Zustand. Die Decke wird nach Öffnen der Verpackung und Ausbreiten durch den Sauerstoff der Umgebungsluft automatisch aktiviert. Dieser oxidiert das Eisen(II)Pulver in einer exogenen Reaktion, wobei Wärme produziert wird. Gemäß den Herstellerangaben beträgt die Aktivierungszeit 30 Minuten, in der eine durchschnittliche Temperatur von $40^{\circ} \mathrm{C}$ erreicht wird und für zehn Stunden erhalten bleiben soll. Dabei sollen 
die Decken eine Maximaltemperatur von $43^{\circ} \mathrm{C}$ nicht überschreiten (Gebrauchsanweisung Mölnlycke Health Care GmbH 2012, Seitenabruf am 01.09.2014, siehe Anhang).

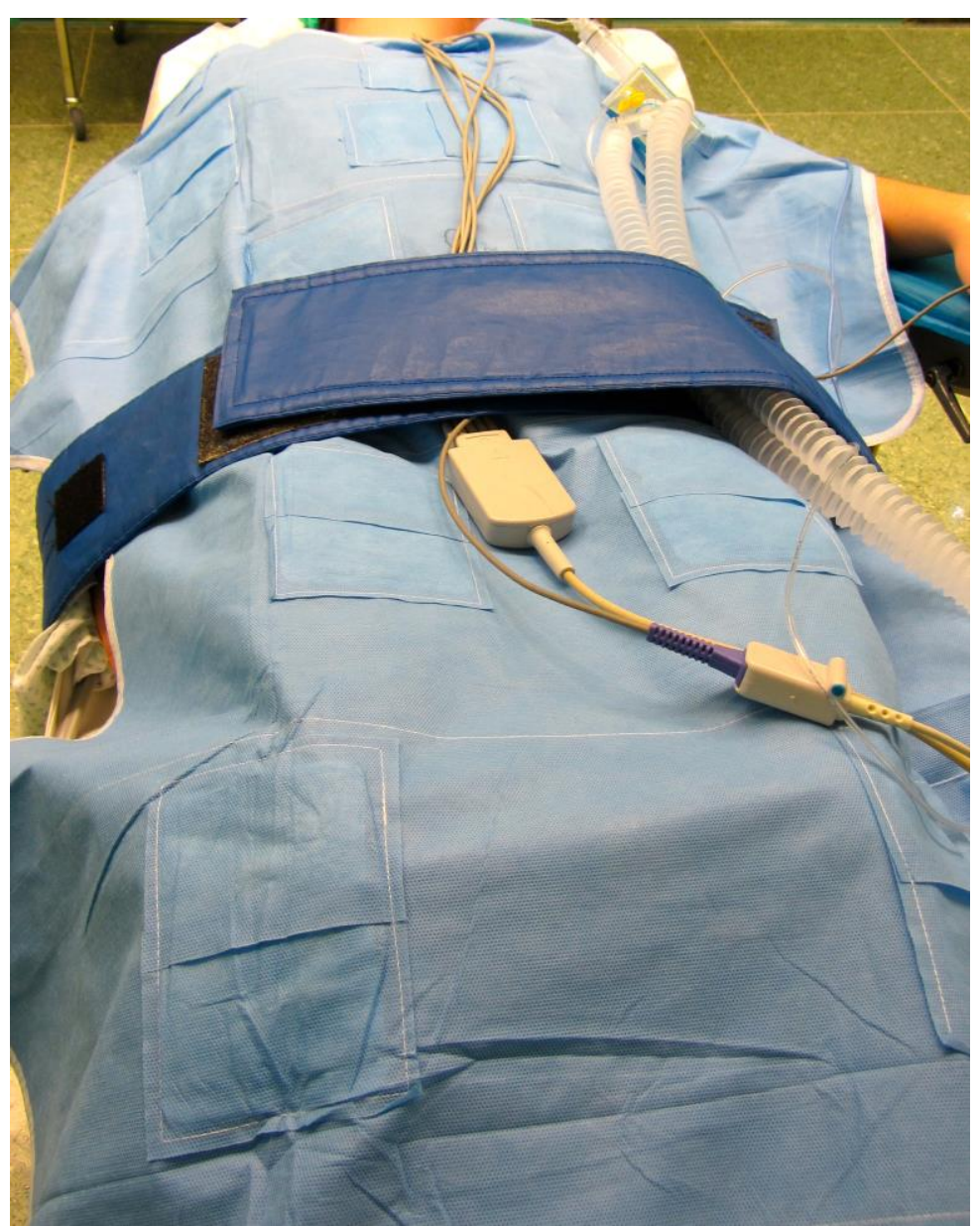

Abbildung 1: Wärmedecke Barrier ${ }^{\circledR}$ Easywarm ${ }^{\circledR}$

Um die Decken auf ihre Wärmeproduktion zu untersuchen, wurde an vier Decken exemplarisch die Oberflächentemperatur gemessen. Dafür wurde ein selbstklebendes Hauttemperaturthermometer (Mon-a-Therm 400TM, Covidien Deutschland $\mathrm{GmbH}$, Neustadt/Donau, Deutschland) direkt unter einem Wärmepad platziert und die Temperatur für vier Stunden aufgezeichnet. Die Messungen wurden in der ersten Stunde minütlich und danach 15-minütlich dokumentiert und erfolgten bei einer Raumtemperatur von 19 bis $22^{\circ} \mathrm{C}$. 


\subsection{Die Temperaturmessungen}

Die Saaltemperatur wurde überwacht und dabei als Zieltemperatur 19,5 bis $20,5^{\circ} \mathrm{C}$ angestrebt. In einigen Fällen lag diese etwas über bzw. unter dem Zielwert, welches auf die unterschiedliche Leistung der Klimaanlage in den drei verschiedenen HNO-Operationssälen zurückzuführen sein könnte.

Präoperativ wurde die orale Temperatur mittels eines elektronischen Fieberthermometers (Geratherm@ Rapid, Geratherm Medical AG, Geschwenda, Deutschland) ermittelt (Abbildung 2). Das Thermometer wurde mit einem Überzug (Soft Temp®, Intermedica $\mathrm{GmbH}$, Mainz, Deutschland) versehen und gemäß der Bedienungsanleitung in der hinteren sublingualen Tasche positioniert. Die Temperatur wurde bei geschlossenem Mund gemessen und die Messungen wurden dreimal wiederholt. Die Messgenauigkeit wird in der Bedienungsanleitung laut Hersteller mit $+/-0,1^{\circ} \mathrm{C}$ im Bereich von 34 bis $42{ }^{\circ} \mathrm{C}$ angegeben (Geratherm $\odot$ Rapid, Geratherm Medical AG, Geschwenda, Deutschland).

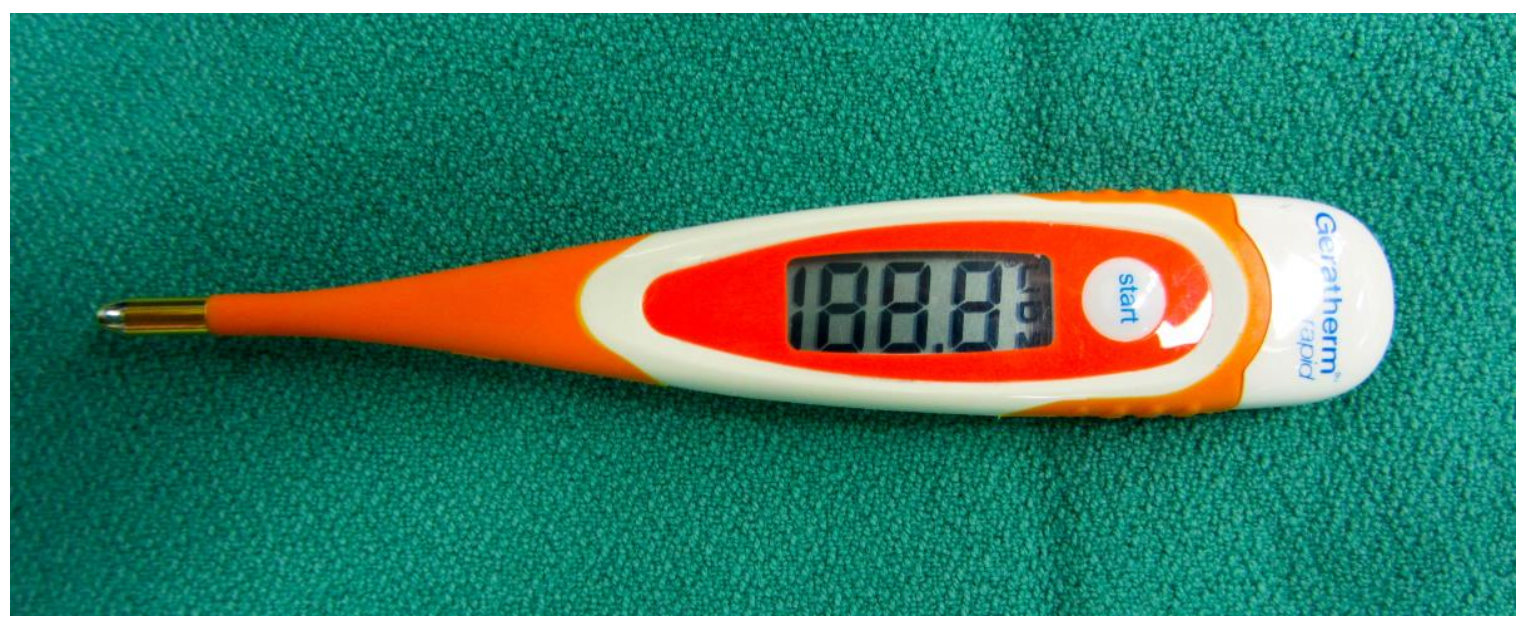

Abbildung 2: Thermometer Geratherm ${ }^{\circledR}$

Nach Narkoseeinleitung erfolgte die Messung der Körperkerntemperatur mithilfe einer ösophagealen Temperatursonde (Rüsch Rektal-/Pharyngeal Temperatursonde, Teleflex Medical GmbH, Kernen, Deutschland) (Abbildung 3). Diese wurde in den Ösophagus eingeführt bis ihre Zählmarkierung bei $35 \mathrm{~cm}$ Mundwinkel lag. Diese Temperatursonde hat laut Hersteller eine Messgenauigkeit von +/$0,2^{\circ} \mathrm{C}$ im Bereich von 25 bis $45^{\circ} \mathrm{C}$ (Rüsch Rektal-/Pharyngeal Temperatursonde, 
Teleflex Medical GmbH, Kernen, Deutschland). Die Temperaturaufzeichnung erfolgte gemäß Studienprotokoll fünf Minuten nach Einführen der Temperatursonde, definiert als Ende der Narkoseeinleitung, sowie bei Hautschnitt intraoperativ alle 15 Minuten und abschließend bei Operationsende.

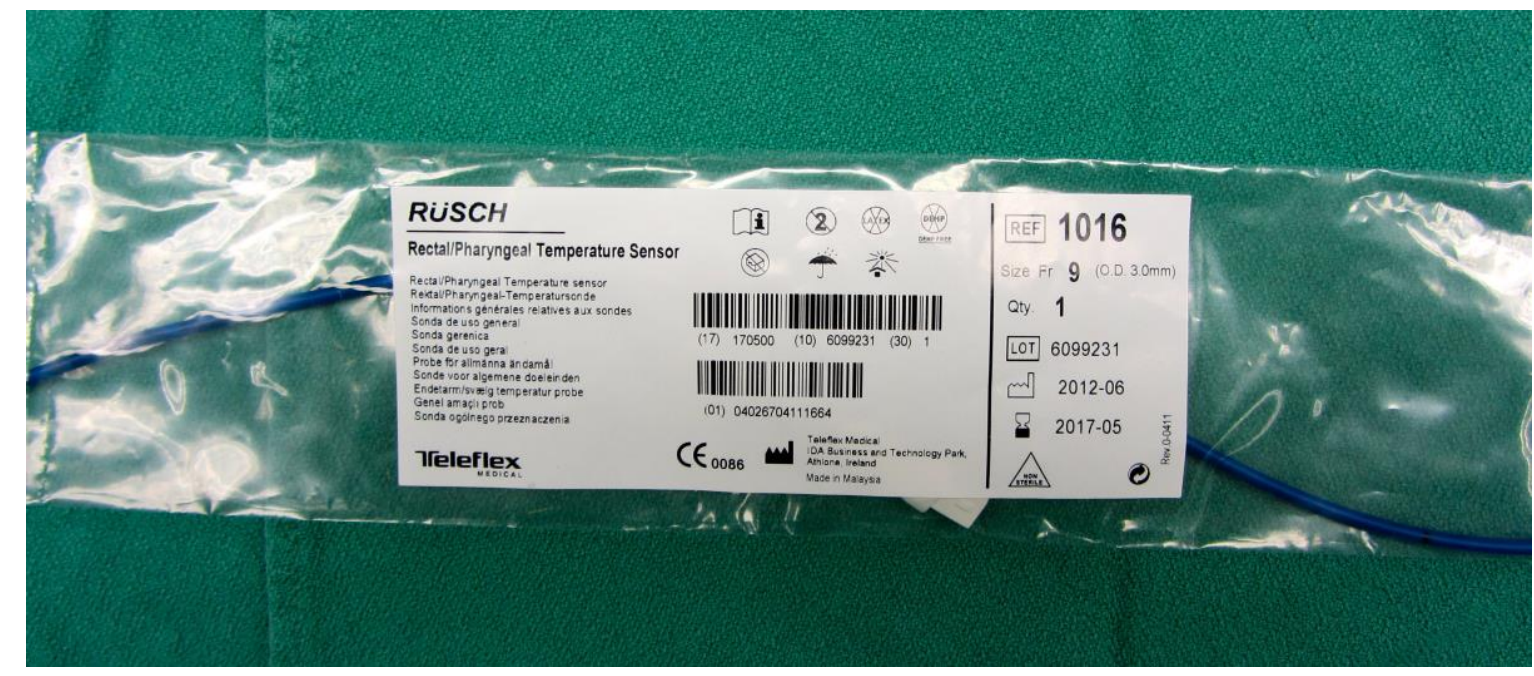

Abbildung 3: Temperatursonde Rüsch®

\subsection{Narkoseführung}

Die Narkoseführung wurde nach der im Bereich der HNO gültigen Standard Operating Procedure durchgeführt. Nach Präoxygenierung über eine dicht sitzende Gesichtsmaske mit $100 \%$ Sauerstoff über drei Minuten erfolgte die Narkoseinduktion mit Remifentanil (Ultiva®, Glaxo Smithkline GmbH \& Co KG, München, Deutschland) mit 0,5 $\mu \mathrm{g}$ pro Kilogramm Körpergewicht pro Minute und einem Propofolbolus (Propofol-Lipuro®, B. Braun AG, Melsungen, Deutschland) von $2 \mathrm{mg}$ pro Kilogramm Körpergewicht über zwei Minuten. Wurde die Sicherung des Atemweges mittels Intubation angestrebt, erfolgte davor die Muskelrelaxierung mit 0,6 mg pro Kilogramm Körpergewicht Rocuronium (Esmeron $\circledast$, MSD Sharp \& Dohme GmbH, München, Deutschland). Wurde eine Larynxmaske zur Atemwegssicherung verwendet, so wurde auf die Relaxierung verzichtet. Zur Aufrechterhaltung der Narkose wurde Remifentanil mit 0,2 $\mu \mathrm{g}$ pro Kilogramm Körpergewicht pro Minute und Propofol mit 4 mg pro Kilogramm Körpergewicht pro Stunde verwendet. 
Das Infusionsregime bestand aus nicht vorgewärmten kristalloiden Infusionen mit Sterofundin $\AA$ 1/1E (Sterofundin $\AA$ Iso Ecoflac, B. Braun, Melsungen, Deutschland) und bei Bedarf mit dem Kolloid Gelafundin® 4 \% (Gelafundin® 4 \% Ecoflac plus, B. Braun AG, Melsungen, Deutschland). Eine spezielle Infusionsgeschwindigkeit sowie Infusionsmenge wurde nicht vorgegeben.

Der Verbrauch an Anästhetika sowie Infusionen wurde quantitativ auf einem papierbasierten Messprotokoll standardisiert erfasst.

\subsection{Parameter Aufwachraum}

Die Verweildauer der Patienten im Aufwachraum sowie die Gabe von Clonidin (Clonidin-ratiopharm ${ }^{\circledR}$, ratiopharm $\mathrm{GmbH}$, Ulm, Deutschland) bei postoperativem Shivering wurden standardisiert auf einem Papierbogen erfasst. Ebenfalls wurde auf dem Dokumentationsbogen notiert, ob die Patienten der Interventionsgruppe eine konventionelle Bettdecke oder anderweitige Wärmemaßnahmen z. B. über konvektive Luftwärmer benötigt oder verlangt haben. Die Dokumentation zusätzlicher Wärmemaßnahmen in Form von konvektiver Wärmezufuhr erfolgte ebenfalls für Patienten der Kontrollgruppe.

\subsection{Fallzahlplanung und statistische Auswertung}

Um für diese Studie die benötigte Anzahl von Patienten abzuschätzen, wurden die Daten früherer Studien herangezogen. In den vorangehenden Unter-suchungen trat bei circa 50 \% der Patienten eine Hypothermie mit einer Körperkerntemperatur unter $36^{\circ} \mathrm{C}$ auf. Unter der Annahme, dass das Patientenkollektiv der Interventionsgruppe eine Hypothermieinzidenz von $20 \%$ aufweist, errechnet sich in der Poweranalyse bei einer Power $(\beta)$ von 0,8 und einem $\alpha$ von 0,05 eine erforderliche Anzahl von 54 Patienten (27 pro Gruppe). Die Anzahl der Patienten wurde auf 40 pro Gruppe erhöht, da sich in vorherigen Studien gezeigt hat, dass circa $25 \%$ aller Patienten aufgrund von Fehleinschätzungen der Operationsdauer post hoc ausgeschlossen werden mussten.

Die statistische Testung wurde mit STATISTICA für Windows 9.0 (StatSoft Inc., Tulsa, OK, USA) durchgeführt. Die Ergebnisse werden als Mittelwert \pm Standardabweichung oder als Median und Interquartil Range angegeben. Ein $p<0,05$ galt als statistisch signifikant. 
In der statistischen Analyse wurden die kategorialen Daten mit dem Fisher'sExact-Test verglichen. Die Untersuchung auf Normalverteilung der kontinuierlichen Daten erfolgte mit dem Kolmogorov-Smirnov-Test. Bei vorliegender Normalverteilung wurde zur statistischen Auswertung der Student's-t-Test angewandt. Nicht normal verteilte Daten wurden mittels Mann-Whitney-U-Test auf statistische Signifikanz getestet. Der zeitliche Verlauf der Körperkerntemperatur wurde mittels Repeated measures analysis of variance (ANOVA) und Post-hocScheffé's-Test untersucht.

Für die Analysen wurden alle Patienten berücksichtigt, die per protocol behandelt wurden und nicht post hoc ausgeschlossen werden mussten. 


\section{Ergebnisse}

\subsection{Patienten}

Das abgebildete Flussdiagramm demonstriert die Daten zum Patienteneinschluss der begutachteten und randomisierten Patienten. Des Weiteren werden die Gruppenzuteilung in die Interventionsgruppe und Kontrollgruppe und die präund postoperativ ausgeschlossenen Patienten mit Ursachen dargestellt. Den Abschluss bilden die eingeschlossenen und der Analyse zugeführten Patienten. In den folgenden Unterpunkten erfolgt die Beschreibung der Daten des Flussdiagramms (Abbildung 4).

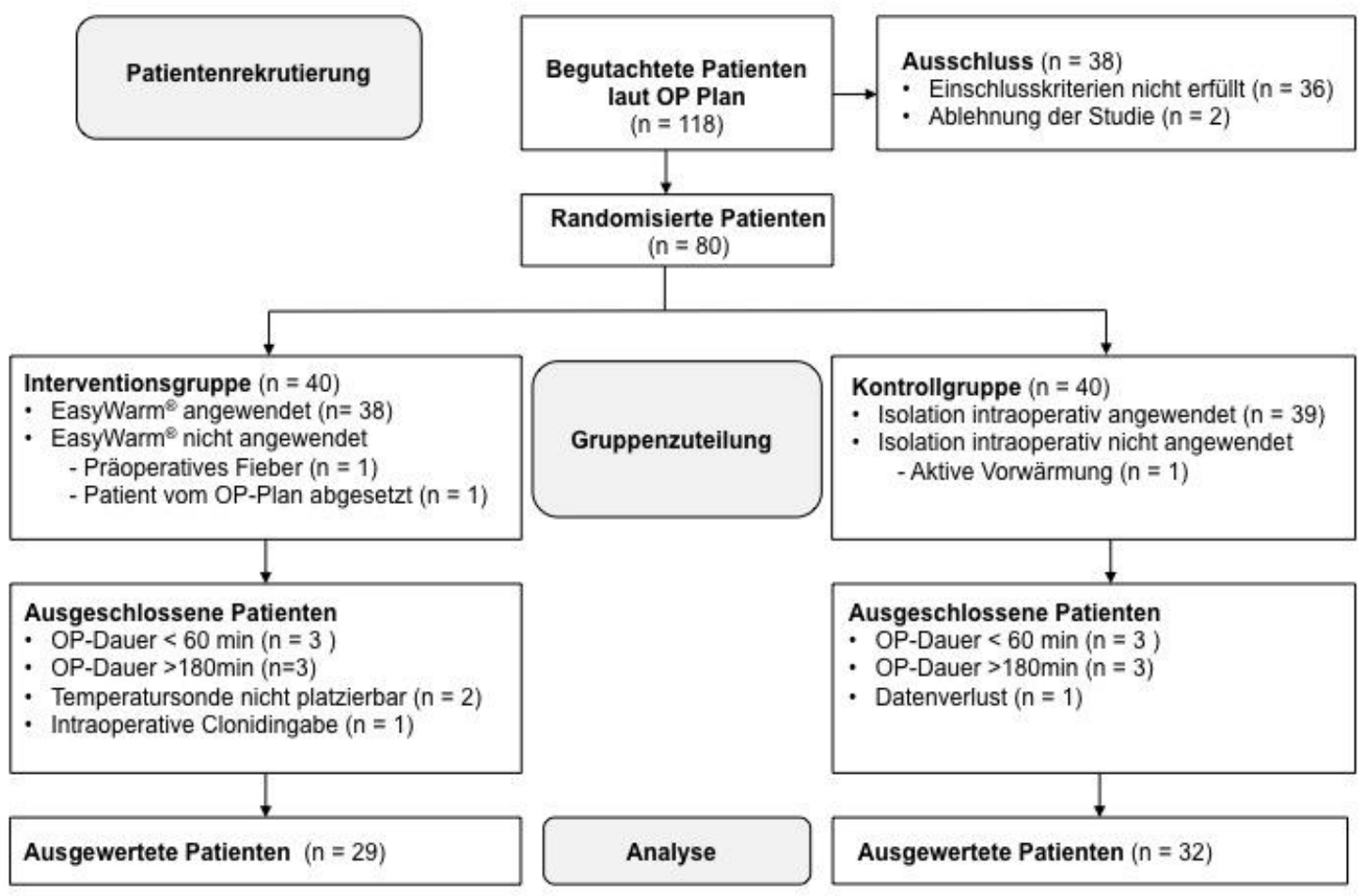

Abbildung 4: Flussdiagramm Patientenkollektiv (modifiziert nach Brandes et al. 2013, S. 138, mit freundlicher Genehmigung von Springer Science + Business Media)

\subsubsection{Patientenscreening}

Das Screening der potentiell eligiblen Patienten erfolgte anhand des Operationplans des Vortages. Patienten im Alter von 18 bis 75 Jahren, welche sich Ope- 
rationen mit einer geplanten Operationsdauer von 60 bis 180 Minuten unterziehen werden, wurden auf weitere Ein- und Ausschlusskriterien untersucht. Dabei mussten die geplanten Operationen eine Platzierung der ösophagealen Temperatursonde ermöglichen, daher wurden Patienten mit geplanter Panendoskopie nicht gescreent. Von diesen 118 gescreenten Patienten mussten 38 ausgeschlossen werden. Davon lehnten zwei Patienten nach Aufklärung die Teilnahme an der Studie ab. 36 Patienten erfüllten die Einschlusskriterien bezüglich des Body Mass Indexes nicht, welcher zwischen 20 und 30 Kilogramm pro Quadratmeter liegen musste.

\subsubsection{Präoperativ ausgeschlossene Patienten}

In beiden Gruppen mussten Studienteilnehmer bereits präoperativ ausgeschlossen werden. In der Interventionsgruppe betraf dies zwei von 40 Patienten. Ein Patient davon wurde vom Operationsplan abgesetzt, der andere wies präoperativ bei den sublingualen Temperaturmessungen bereits Fieber mit einer Körperkerntemperatur von über $38^{\circ} \mathrm{C}$ auf. Bei 38 Patienten der Interventionsgruppe wurde die intraoperative Wärmezufuhr mit der Wärmedecke Barrier ${ }^{\circledR}$ Easywarm ${ }^{\circledR}$ durchgeführt. In der Kontrollgruppe wurde ein Patient von 40 Patienten präoperativ ausgeschlossen, da er vor Narkoseeinleitung im Gegensatz zum im Studienprotokoll festgelegten Vorgehen mit einem konvektiven Wärmesystem vorgewärmt wurde. Die verbleibenden 39 Patienten wurden gemäß Studiendesign mit Isolation durch die Krankenhaussteppdecke versorgt.

\subsubsection{Postoperativ ausgeschlossene Patienten}

Postoperativ mussten ebenfalls in beiden Gruppen Patienten ausgeschlossen werden. Dies betraf neun Patienten der Interventionsgruppe und sieben Patienten der Kontrollgruppe.

In der Interventionsgruppe wurden sechs Patienten aufgrund einer Fehleinschätzung der Operationszeiten ausgeschlossen. Dabei betrug bei drei Patienten die Operationsdauer weniger als 60 Minuten. Bei drei Patienten betrug diese mehr als 180 Minuten. Bei zwei Patienten konnte nach Narkoseeinleitung keine Temperatursonde platziert werden, da die Operation dieses doch nicht erlaubte. Ein Patient erhielt intraoperativ Clonidin intravenös, um eine permissive Hypotonie zu erreichen. Da dies den Wärmehaushalt beeinflussen kann, wurde der Patient ausgeschlossen.

In der Kontrollgruppe wurden ebenfalls sechs Patienten aufgrund von Fehleinschätzungen der Operationszeiten ausgeschlossen. Bei drei Patienten betrug die Operationsdauer weniger als 60 Minuten. Bei drei Patienten betrug diese mehr 
als 180 Minuten. Bei einem Patienten trat ein Datenverlust auf, sodass dieser nicht in die Analysen eingeschlossen werden konnte.

\subsubsection{Eingeschlossene Patienten}

Von den 80 randomisierten Patienten konnten 29 Patienten der Interventionsgruppe und 32 Patienten der Kontrollgruppe in die Analysen eingeschlossen werden.

Tabelle 1 zeigt für die beiden Gruppen die Patientendaten und die perioperativen Verlaufsdaten als Mittelwert mit Standardabweichung bei Alter, Körpergröße, Körpergewicht, Temperatur im Operationssaal, Körperkerntemperatur vor der Narkoseeinleitung, Operations- und Narkosedauer. Die Vorwärmdauer wird als Median mit Interquartilsabstand, die Geschlechterverteilung als absolute Anzahl angegeben. Wenn möglich, wurde der berechnete p-Wert abgebildet. Die Erläuterungen zu Tabelle 1 werden in den nächsten Abschnitten dargestellt. 
Tabelle 1: Patientendaten und perioperative Verlaufsdaten

- Darstellung der Werte als Mittelwert und Standardabweichung (MW $\pm S D$ ), als Median und Interquartilsabstand [Interquartilsabstand] oder als Anzahl der Patienten

Variable

Interventions- Kontroll-

p-Wert

gruppe $(n=29)$ gruppe $(n=32)$

\begin{tabular}{|c|c|c|c|}
\hline \multicolumn{4}{|l|}{ Biometrische Daten } \\
\hline Alter (Jahre) & $45 \pm 17$ & $44 \pm 15$ & 0,33 \\
\hline Geschlecht (m/w) & $18 / 11$ & $20 / 12$ & 1,0 \\
\hline Körpergröße (cm) & $173 \pm 12$ & $175 \pm 10$ & 0,46 \\
\hline Körpergewicht (kg) & $76 \pm 15$ & $80 \pm 13$ & 0,49 \\
\hline Temperaturen & & & \\
\hline $\begin{array}{l}\text { Körperkerntemperatur vor } \\
\text { Narkoseeinleitung }\left({ }^{\circ} \mathrm{C}\right)\end{array}$ & $36,1 \pm 0,3$ & $36,2 \pm 0,3$ & 0,84 \\
\hline Operationssaal $\left({ }^{\circ} \mathrm{C}\right)$ & $20 \pm 1$ & $20 \pm 1$ & 0,85 \\
\hline Zeiten & & & \\
\hline Vorwärmdauer (min) & 85 [50-135] & - & - \\
\hline Operationsdauer (min) & $91 \pm 26$ & $94 \pm 31$ & 0,37 \\
\hline Narkosedauer (min) & $106 \pm 28$ & $108 \pm 32$ & 0,47 \\
\hline
\end{tabular}

\subsubsection{Vergleich der biometrischen Daten}

Die biometrischen Daten der Patienten waren bezüglich Alter, Geschlecht, Körpergröße und Körpergewicht vergleichbar und statistisch nicht signifikant unterschiedlich.

Die Patienten der Interventionsgruppe waren bei einer Standardabweichung von 17 Jahren im Mittel 45 Jahre alt. Bei der Kontrollgruppe ergab sich bei einer Standardabweichung von 15 Jahren ein Mittelwert von 45 Jahren. In der Interventionsgruppe zeigte sich eine Geschlechterverteilung von 18 männlichen und 
elf weiblichen Teilnehmern. Bei der Kontrollgruppe fanden sich 20 männliche und zwölf weibliche Teilnehmer. Die Patienten der Interventionsgruppe wiesen bezüglich der Körpergröße einen Mittelwert von $173 \mathrm{~cm}$ mit einer Standardabweichung von $12 \mathrm{~cm}$ auf. Ähnliche Werte zeigten die Patienten der Kontrollgruppe bei der Körpergröße mit einem Mittelwert von $175 \mathrm{~cm}$ und einer Standardabweichung von $10 \mathrm{~cm}$. Die Patienten der Interventionsgruppe hatten bei einer Standardabweichung von 15 Kilogramm ein mittleres Gewicht von 76 Kilogramm. Die Patienten der Kontrollgruppe wogen im Mittel 80 Kilogramm bei einer Standardabweichung von 13 Kilogramm.

\subsubsection{Vergleich der Operations- und Narkosedauer}

Die Dauer der Narkose sowie der Operation war zwischen den beiden Gruppen vergleichbar und statistisch nicht signifikant unterschiedlich.

In der Interventionsgruppe betrug die Narkosedauer im Mittel 106 Minuten bei einer Standardabweichung von 28 Minuten. In der Kontrollgruppe betrug sie 108 Minuten (Mittelwert) bei einer Standardabweichung von 32 Minuten. Die Operationsdauer betrug in der Interventionsgruppe 91 Minuten (Mittelwert) mit einer Standardabweichung von 26 Minuten. In der Kontrollgruppe betrug sie 94 Minuten (Mittelwert) mit einer Standardabweichung von 31 Minuten.

\subsubsection{Vergleich der Vorwärmdauer in der Interventionsgruppe}

Die Vorwärmdauer mit der Wärmedecke Barrier® Easywarm ${ }^{\circledR}$ vor Narkoseeinleitung betrug im Median 85 Minuten mit einem Interquartilsabstand von 50 bis 135 Minuten.

\subsection{Temperaturverlauf der Decken}

An vier Decken wurden exemplarische Messungen der Oberflächentemperatur direkt unter einem Wärmepad vorgenommen und über vier Stunden aufgezeichnet (Abbildung 5). Dabei erreichten zwei Decken nach 30 Minuten an-nähernd inre Maximaltemperaturen, welche im weiteren Verlauf der Messungen nur noch um 0,4 bis $0,6^{\circ} \mathrm{C}$ anstiegen. Bei den anderen beiden Decken stieg die Temperatur der Wärmepads nach 30 Minuten dagegen noch um 2,2 bis 2,4 ${ }^{\circ} \mathrm{C}$ an, bis sie ihren Maximalwert während der Messungen erreichten. Die höchsten erzielten Deckentemperaturen variierten allerdings in den vorliegenden Aufzeichnungen zwischen 36,4 und $42{ }^{\circ} \mathrm{C}$. 


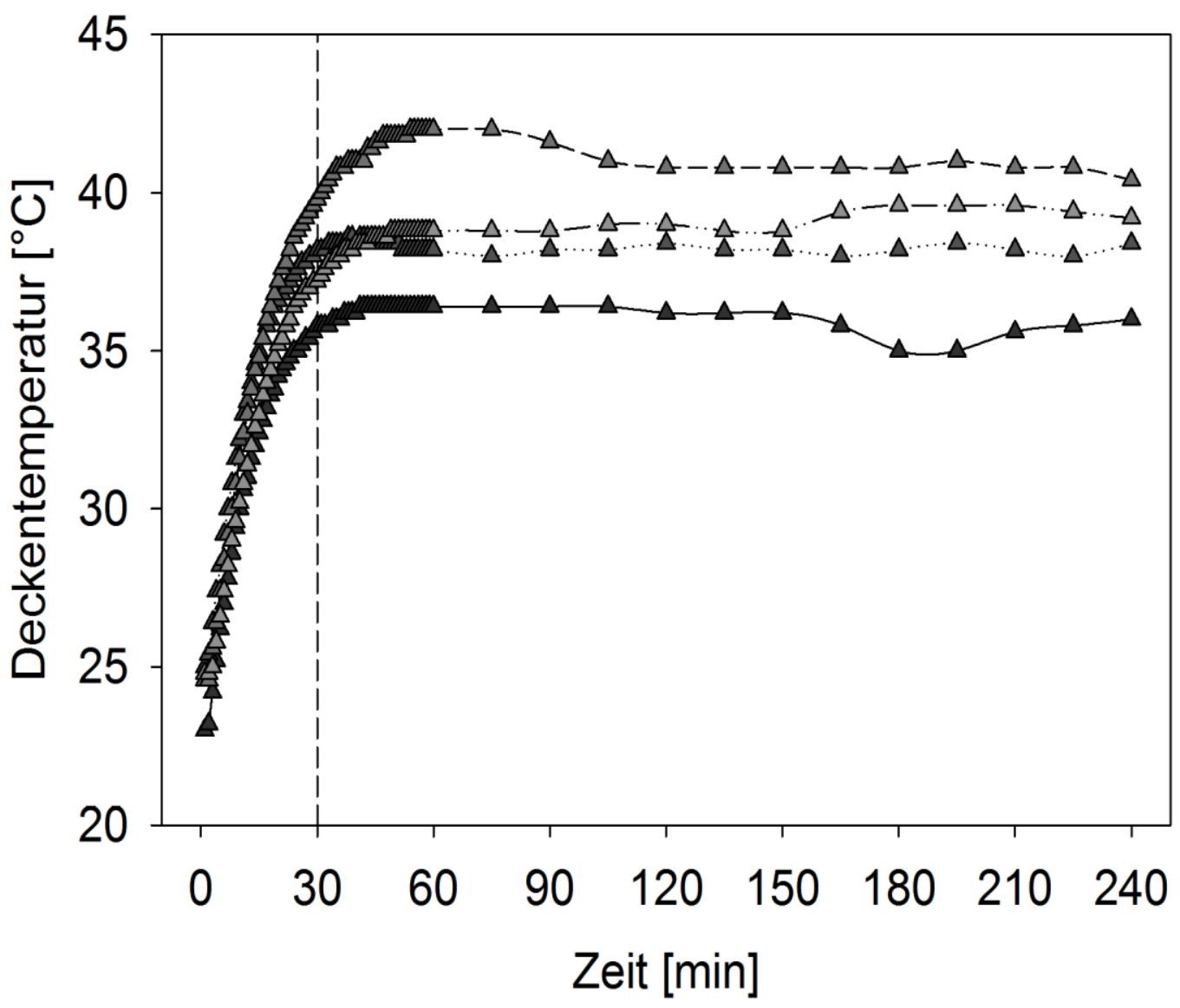

Abbildung 5: Vier exemplarische Messungen der Temperaturentwicklung nach Aktivierung der Decken (modifiziert nach Brandes et al. 2013, S. 141, mit freundlicher Genehmigung von Springer Science + Business Media)

\subsection{Temperaturen}

\subsubsection{Temperatur im Operationssaal}

Die Temperaturen im Operationssaal wurden überwacht und zeigten bei den Gruppen keinen signifikanten Unterschied $(p=0,85)$. Sie betrugen im Mittel $20^{\circ} \mathrm{C}$ mit einer Standardabweichung von $1{ }^{\circ} \mathrm{C}$ (Tabelle 1).

\subsubsection{Präoperative Temperaturen}

Bereits vor der Narkoseeinleitung wiesen Patienten aus beiden Gruppen Körperkerntemperaturen unter $36{ }^{\circ} \mathrm{C}$ auf. Diese wurden bei vier von 29 Patienten der Interventionsgruppe und bei sechs von 32 Patienten der Kontrollgruppe aufgezeichnet. Dementsprechend waren $14 \%$ der vorgewärmten und $19 \%$ der nur mit Isolation versorgten Patienten hypotherm vor Narkoseeinleitung. Insgesamt 
zeigte sich zwischen den Gruppen kein statistisch signifikanter Unterschied in der Inzidenz präoperativer Hypothermie ( $p=0,73$; Tabelle 1).

\subsubsection{Intraoperative Temperaturen}

Der intraoperative Temperaturverlauf der Körperkerntemperaturen wurde mittels ANOVA analysiert und zeigte zu keinem Zeitpunkt Unterschiede in der Körperkerntemperatur der beiden Patientengruppen. Ebenso zeigte sich kein statistisch signifikanter Unterschied beim Auftreten intraoperativer Hypothermie in beiden Gruppen $(p=0,44)$. Dabei lag die Inzidenz für intraoperative Hypothermie in der Interventionsgruppe mit $41 \%$ (zwölf von 29 Patienten) sogar höher als in der Kontrollgruppe mit $31 \%$ (zehn von 32 Patienten).

Die Abbildung 6 zeigt den Temperaturverlauf der Körperkerntemperaturen präund intraoperativ als Mittelwert und Standardabweichung bei den beiden Gruppen.

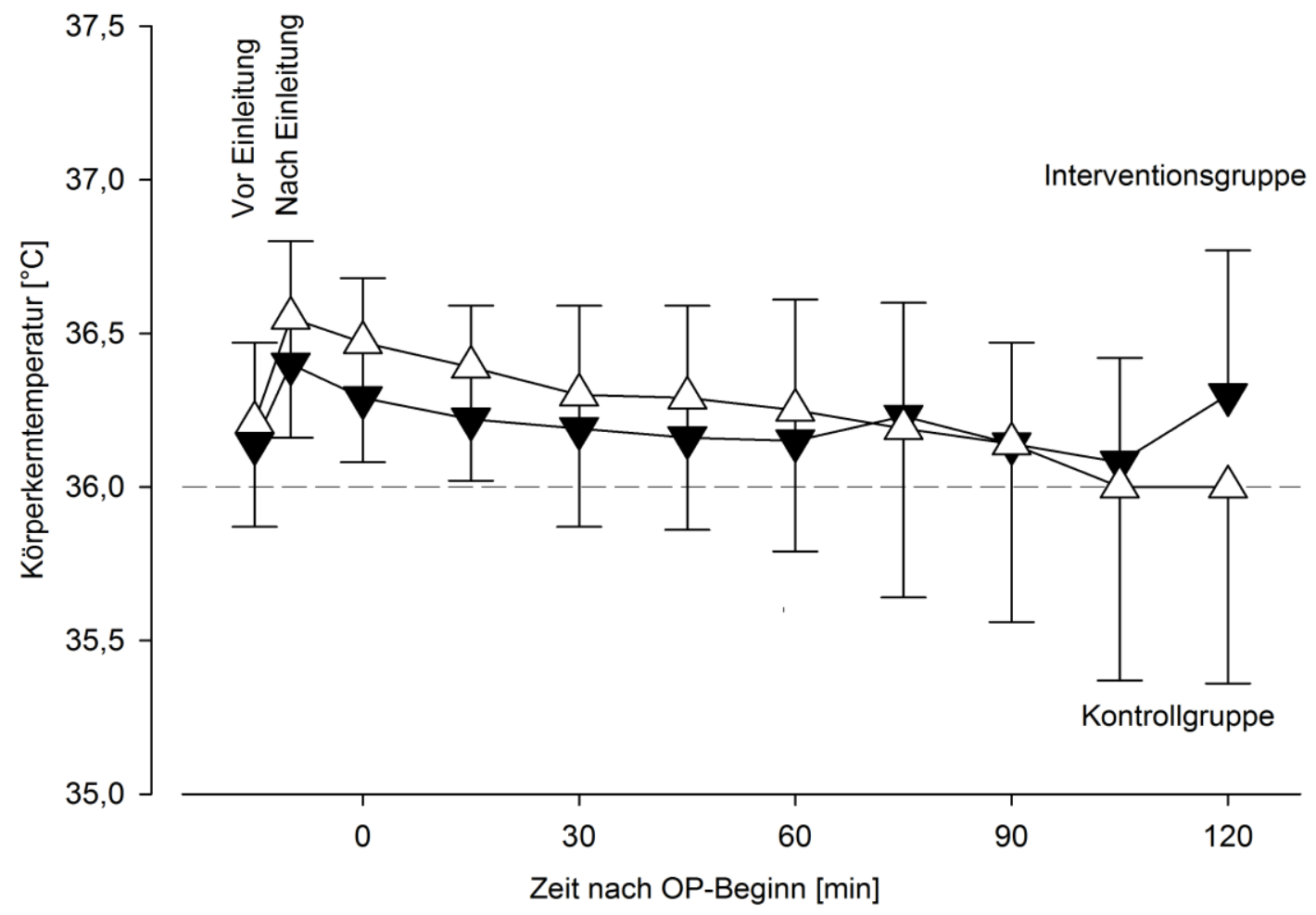

Abbildung 6: Mittelwerte und Standardabweichungen der perioperativen Körperkerntemperaturen

(modifiziert nach Brandes et al. 2013, S. 141, mit freundlicher Genehmigung von Springer Science + Business Media)

Geschlossene Dreiecke: Interventionsgruppe, offene Dreiecke: Kontrollgruppe 


\subsubsection{Postoperative Temperaturen}

Das Auftreten von Hypothermie bei den Patienten war auch postoperativ in beiden Gruppen hoch. Dabei wiesen zwölf von 29 Patienten in der Interventionsgruppe und neun von 32 Patienten in der Kontrollgruppe nach der Operation Temperaturen unter $36{ }^{\circ} \mathrm{C}$ auf. Somit lag die Hypothermieinzidenz postoperativ bei $41 \%$ in der Interventionsgruppe und bei $28 \%$ in der Kontrollgruppe. Insgesamt zeigte sich kein statistisch signifikanter Unterschied in der Inzidenz postoperativer Hypothermie zwischen den Gruppen ( $p=0,3)$.

Zusammenfassend kann festgestellt werden, dass HNO-Patienten perioperativ mit circa $36 \%$ in hohem Maß von unerwünschter Hypothermie betroffen waren. Die Inzidenz perioperativer Hypothermie war in beiden Gruppen mit 31 bis $41 \%$ klinisch relevant hoch, jedoch statistisch nicht signifikant unterschiedlich. Die Wärmedecke Barrier ${ }^{\circledR}$ Easywarm ${ }^{\circledR}$ führte nicht zu einer Reduktion des Auftretens perioperativer Hypothermie in der Interventionsgruppe.

\subsection{Parameter im Aufwachraum}

\subsubsection{Verweildauer im Aufwachraum}

Bezüglich der Verweildauer im Aufwachraum gab es keinen Unterschied zwischen den beiden Patientengruppen $(p=0,49)$. Die Patienten der Interventionsgruppe verbrachten 110 Minuten (Mittelwert) bei einer Standardabweichung von 36 Minuten und die Patienten der Kontrollgruppe 114 Minuten (Mittelwert) bei einer Standardabweichung von 32 Minuten im Aufwachraum (Tabelle 1).

\subsubsection{Auftreten von Shivering}

Trat im Aufwachraum postoperatives Kältezittern (Shivering) auf, wurde dieses bei vier von 29 Patienten in der Interventionsgruppe und bei acht von 32 Patienten der Kontrollgruppe mit fraktionierter Gabe von Clonidin behandelt. Somit erhielten $14 \%$ der Patienten in der Interventionsgruppe und $25 \%$ der Patienten in der Kontrollgruppe postoperativ Clonidin. Die postoperative Clonidingabe war statistisch nicht signifikant unterschiedlich $(p=0,34)$.

\subsubsection{Akzeptanz der Wärmedecke}

Ein Großteil der Patienten der Interventionsgruppe wünschte sich postoperativ eine andere Decke (elf von 29 Patienten), da die Wärmedecke Barrier® Easywarm ${ }^{\circledR}$ postoperativ als unangenehm empfunden wurde. In einem Fall trat Schwitzen unter der Decke auf $(n=1)$, zumeist froren jedoch die Patienten mit der 
Wärmedecke allein $(n=10)$. Des Weiteren wurden fünf Patienten der Interventionsgruppe postoperativ mit konvektiver Luftwärmung gewärmt. Dies benötigten dagegen nur drei Patienten der Kontrollgruppe. Somit akzeptierten 16 von 29 Patienten der Interventionsgruppe (55\%) die Wärmedecke Barrier $\AA$ Easywarm ${ }^{\circledR}$ postoperativ nicht.

\subsubsection{Nebenwirkungen der Wärmetherapie}

Bei keinem Patienten wurden unerwünschte schwere Nebenwirkungen durch den Einsatz der Wärmedecke beobachtet. Es traten keine Hautläsionen oder Verbrennungen auf. 


\section{Diskussion}

\subsection{Entwicklung der Studienidee}

Da das Auftreten von Hypothermie während einer Allgemeinanästhesie mit einer Vielzahl von Komplikationen (Sessler 2001) sowie dadurch entstehenden Kosten (Mahoney und Odom 1999) verbunden ist, soll perioperative Hypothermie durch ein adäquates Wärmemanagement vermieden werden (NICE 2008). Auf dem Markt stehen unterschiedliche Wärmeprotektionsverfahren zur Verfügung, die in verschiedenen Studien auf ihre Effektivität untersucht und mit-einander verglichen worden sind (Moola und Lockwood 2011). Da sich die bisher untersuchten Wärmeprotektionsverfahren z. T. stark in der Effektivität der Prävention perioperativer Hypothermie unterscheiden (Galvao et al. 2010), soll auch die neue Wärmedecke Barrier ${ }^{\circledR}$ Easywarm ${ }^{\circledR}$ erstmals in einer kontrolliert prospektiv randomisierten Studie untersucht werden.

Um das Potential der Wärmedecke Barrier $\AA$ Easywarm $\AA$ zu ermitteln, wurde versucht optimale Studienbedingungen für das Produkt zu ermöglichen. So wurde zum einen die Möglichkeit des Prewarmings durch die Wärmedecke Barrier® Easywarm $\AA$ in Form von mindestens 30-minütiger aktiver Vorwärmung in der Interventionsgruppe angewandt, während die Kontrollgruppe kein Prewarming erhielt. Daneben bietet die Auswahl der Patientengruppe, welche sich HNOchirurgischen Eingriffen mittlerer Dauer unterzieht, die Möglichkeit einer optimalen prä- als auch intraoperativen Wärmezufuhr, da fast die ganze Körperoberfläche hierfür genutzt werden kann. Des Weiteren sind bei diesen Eingriffen die Wärmeverluste über das kleine Operationsgebiet sowie wegen einer moderaten Volumensubstitution aufgrund der minimalen Blutverluste gering.

Obwohl die oben genannten Aspekte Gründe dafür darstellen, dass HNOPatienten nur ein geringes Risiko für die Entwicklung perioperativer Hypothermie haben müssten, führt dennoch das Durchführen einer Allgemeinanästhesie selber zu einem Risiko für die Entstehung perioperativer Hypothermie (Sessler 1997).

Es gibt wenige Studien in der die tatsächliche Inzidenz perioperativer Hypothermie bei HNO-Patienten untersucht wird. Da in bisherigen Studien auch bei HNOPatienten Hypothermie mit 30 bis $60 \%$ in relevantem Maße auftritt (Agrawal et al. 2003, Karalapillai et al. 2009, Karalapillai et al. 2013) soll zum einen die Inzidenz von Hypothermie bei HNO-chirurgischen Eingriffen in dieser Studie untersucht sowie die Effektivität der Vermeidung perioperativer Hypothermie durch 
das neue konduktive Wärmesystem Barrier® Easywarm® überprüft werden. Ziel ist es die Datenlage zu festigen um als Konsequenz zukünttig das Wärmemanagement in der klinischen Praxis bei HNO-chirurgischen Patienten optimieren zu können.

\subsubsection{Potentielle Vorteile der Wärmedecke Barrier® Easywarm®}

Die Wärmedecke Barrier ${ }^{\circledR}$ Easywarm ${ }^{\circledR}$ bietet eine Vielzahl theoretischer Vorteile, um dem Patienten in der gesamten perioperativen Phase eine aktive Wärmezufuhr zu ermöglichen. Zu diesen Vorteilen zählt, dass sie als Einmalartikel unabhängig vom Ort flexibel einsetzbar ist. Das heißt, dass auch auf peripheren Stationen oder in der Wartezone keine zusätzlichen Wärmegeräte oder Wärmedecken angeschafft, in ausreichendem Maße vorgehalten und gelagert werden müssen. Die Wärmedecke Barrier® Easywarm® wird zu zehn Stück in einem Karton geliefert und kann so platzsparend gelagert werden (www.molnlycke.de/patientenwarmemanagement/easywarm/ Seitenabruf am 01.09.2014). Des Weiteren ist sie einfach zu bedienen, da sie durch Öffnen der Verpackung und Ausbreiten der Decke vom Patienten selber aktiviert werden kann. Daher reduziert sich die notwendige Schulung des Personals außerhalb des Operationsbereiches auf ein Minimum, da keine Einweisung und kein Training wie bei der Anwendung anderer Wärmesysteme nötig ist. Da die Wärmedecke Barrier® Easywarm ${ }^{\circledR}$ ohne Strom Wärme generiert, kann hierüber der Patient während der gesamten perioperativen Phase gewärmt werden, insbesondere auch während der Transportphase in den Operationssaal. In diesen beiden Punkten weist das Wärmesystem Barrier® Easywarm ${ }^{\circledR}$ gegenüber anderen existierenden Vorwärmesystemen wie z. B. einem konvektiven Wärmehemd (Andrzejowski et al. 2008) oder einer elektrische Wärmematte (Wong et al. 2007) theoretische Vorteile auf. Da die Barrier® Easywarm® Decke laut Hersteller bis zu zehn Stunden Wärme liefert (Gebrauchsanweisung Barrier® Easywarm®, Mönlycke Healthcare $\mathrm{GmbH}$, Erkrath, Deutschland 2012, Seitenabruf am 01.09.2014, siehe Anhang), sollte diese aktive Wärmedauer für die meisten Operationen über die gesamte perioperative Zeit ausreichend sein. Des Weiteren ist der Einsatz der Decke bei verschiedenen Operationen denkbar, da sie in unterschiedlichen Varianten auf den Patienten gelegt werden kann (Gebrauchsanweisung Barrier® Easywarm®, Mönlycke Healthcare GmbH, Erkrath, Deutschland 2012, Seitenabruf am 01.09.2014, siehe Anhang).

\subsubsection{Potentielle Nachteile der Wärmedecke Barrier® Easywarm®}

Als potentieller Nachteil des Wärmesystems Barrier® Easywarm® ist die lange Aktivierungszeit von 30 Minuten anzusehen, welche einen zeitlich organisierten 
Einsatz nötig macht. Wird die Decke allerdings bereits auf der Normalstation eingesetzt, sollte die Aktivierungszeit ausreichen, bis der Patient per Transport und ggf. weiterer Wartezeit in der Wartezone im Operationssaal ankommt. So beträgt in dieser Studie die mittlere Vorwärmzeit 85 Minuten. Als größerer Nachteil ist die fehlende Steuerbarkeit der Temperaturregelung und -kontrolle anzusehen, da dadurch eine für den individuellen Patienten optimierte Wärmezufuhr nicht ermöglicht wird. Durch eine vom Patienten selbständig zu steuernde Wärmezufuhr könnte der Patientenkomfort zudem zusätzlich gesteigert werden (O'Brien et al. 2010).

\subsection{Interpretation der Ergebnisse}

\subsubsection{Klinische Interpretation der Ergebnisse}

In dieser prospektiv randomisierten kontrollierten Studie an HNO-Patienten, die sich HNO-chirurgischen Eingriffen mittlerer Dauer unterzogen, ergibt sich eine Gesamtinzidenz intraoperativer Hypothermie von 36,1 \%. Ähnliche intraoperative Inzidenzen werden sowohl in einer früheren Studie mit einer Gesamtinzidenz von $40 \%$ an einem ähnlichen Patientenkollektiv in dem gleichen Krankenhaus (Perl et al. 2012) als auch bei großen multizentrischen Untersuchungen gefunden (Karalapillai et al. 2009, Karalapillai et al. 2013). In einer Studie an über 50.000 Patienten zum Auftreten von Hypothermie in den ersten 24 Stunden nach Aufnahme auf die Intensivstation zeigt sich eine Inzidenz von 45,8 \% nach HNOchirurgischen und plastischen Eingriffen, welche in dieser Größenordnung auch mit Inzidenzen von $45,7 \%$ bei orthopädischen und von $44,5 \%$ bei allgemeinchirurgischen Operationen vergleichbar ist (Karalapillai et al. 2013). In einer früheren Untersuchung von Karalapillai et al. (2009) an über 5.000 Patienten weisen $31 \%$ der Patienten nach HNO-chirurgischen Eingriffen bei Aufnahme auf die Intensivstation Temperaturen von unter $36^{\circ} \mathrm{C}$ auf, verglichen mit $35 \%$ nach allgemeinchirurgischen und $32 \%$ nach unfallchirurgischen Operationen in diesem $\mathrm{Pa}$ tientenkollektiv (Karalapillai et al. 2009). Allerdings sind HNO-chirurgische Eingriffe, die postoperativ auf einer Intensivstation betreut werden, schwerwiegender und bieten aufgrund der längeren Operationsdauer, der größeren Invasivität des Eingriffes und der höheren Flüssigkeits- und Blutverluste ein größeres Risiko für Hypothermie. Somit ist zu erwarten, dass die Patienten nach ausgedehnten HNO-chirurgischen Eingriffen ebenfalls ein hohes Risiko für Hypothermie haben wie postoperativ intensivpflichtige Patienten nach unfall- und allgemeinchirurgischen Operationen. Daher kann zusammenfassend gesagt werden, dass die in 
dieser Studie erfasste Inzidenz von $36 \%$ Hypothermie bei HNO-chirurgischen Patienten sowohl in einer im Patientenkollektiv und Studiendesign vergleichbaren Studie gefunden wurde als auch im Mittelfeld der Inzidenzen von multizentrischen Studien mit einem zahlenstarken Patientenkollektiv nach größeren Operationen liegt. Von dieser Größenordnung abweichende Daten beschreiben allein Agrawal et al. (2003), die eine Hypothermierate von insgesamt $60 \%$ an diesem Patientenkollektiv finden. Da ein suffizientes Wärmemanagement das Auftreten von Hypothermie reduzieren kann, müssen die vorliegenden Studien unter dem Aspekt der erfolgten Wärmemaßnahmen in der Kontroll- und Interventionsgruppe betrachtet werden. Die Inzidenz für Hypothermie in der Interventionsgruppe hängt unter anderem von der Effektivität des eingesetzten Wärmesystems ab, sodass sich beim Einsatz konvektiver Wärmesysteme $52 \%$ (Agrawal et al. 2003), beim Einsatz einer neuen konduktiven Wärmematte in Kombination mit Isolation durch eine Bettdecke $20 \%$ (Perl et al. 2012) und bei der konduktiven Wärmedecke Barrier ${ }^{\circledR}$ Easywarm ${ }^{\circledR} 41 \%$ hypotherme Patienten in den Interventionsgruppen finden. Aber auch die angewendeten Wärmeprotektionsverfahren in den Kontrollgruppen unterscheiden sich bei den Studien. So sind beim Einsatz eines Baumwolllakens $65 \%$ (Agrawal et al. 2003) und beim Einsatz einer besser isolierenden Bettdecke wie in dieser Studie 31 \% bzw. 40 \% (Perl et al. 2012) der Patienten in der Kontrollgruppe perioperativ hypotherm. Dabei weist eine Bettdecke Isolationswerte von 1,29 clo auf und kann damit die Wärmeverluste über die exponierte Haut besser reduzieren als ein Bettlaken mit 0,17 clo (Brauer et al. 2004c).

Zudem unterscheiden sich die Studien in ihrem Studiendesign bezüglich der Art und Dauer der Eingriffe sowie der zugeführten Infusionsmengen. So beträgt die durchschnittliche Anästhesiedauer in dieser Studie 106 Minuten und ist damit ähnlich lang wie in der Studie von Perl et al. (2012) mit 120 Minuten. Demgegenüber untersuchen Agrawal et al. (2003) größere HNO-Operationen mit einer Dauer von 195 bis 285 Minuten. Dabei zeigt sich auch ein Unterschied in der Menge der applizierten Volumensubstitution von 2100 bis $2900 \mathrm{ml}$ verglichen mit 740 bis $775 \mathrm{ml}$ in dieser Studie. Dies mag einen weiteren Einfluss auf die höhere Gesamtinzidenz von Hypothermie in der Studie von Agrawal et al. (2003) gehabt haben, da die Zufuhr großer ungewärmter Infusionsmengen einen negativen Teil zur Wärmebilanz beiträgt (Sessler 2001).

Des Weiteren sollte man die absoluten Hypothermieinzidenzen in der Interventions- und Kontrollgruppe aus verschiedenen Studien nur im Zusammenhang mit der Gesamtinzidenz der Hypothermie vergleichen. Sonst müsste man schließen, 
dass die Wärmedecke Barrier ${ }^{\circledR}$ Easywarm ${ }^{\circledR}$ besser in der Reduktion perioperativer Hypothermie ist als konvektive Luftwärmer. Allerdings zeigte sich bei dem Einsatz konvektiver Luftwärmer bei Operationsende eine um $0,4{ }^{\circ} \mathrm{C}$ signifikant höhere Temperatur als in der Kontrollgruppe (Agrawal et al. 2003). Dagegen führt der Einsatz der konduktiven Wärmedecke Barrier ${ }^{\circledR}$ Easywarm ${ }^{\circledR}$ zu keinem Zeitpunkt zu einem Unterschied in den Körperkerntemperaturen der Interventionsund Kontrollgruppe. Daher kann die Hypothese, dass der Einsatz des neuen konduktiven Wärmesystems Barrier ${ }^{\circledR}$ Easywarm ${ }^{\circledR}$ zu einer signifikanten Reduktion perioperativer Hypothermie führt, in dieser Studie nicht bestätigt werden. Werden die Hypothermieinzidenzen in den einzelnen perioperativen Phasen verglichen, sind präoperativ $14 \%$ in der Interventionsgruppe und $19 \%$ in der Kontrollgruppe hypotherm, was statistisch nicht signifikant ist. Diese Ergebnisse sind vermutlich auf die lange Prewarming Phase in der Interventionsgruppe von mindestens 30 Minuten, jedoch im Mittel 85 Minuten zurückzuführen. Allerdings zeigt sich dieser Zusammenhang nicht bei Betrachtung der präoperativen Körperkerntemperaturen, die in der Interventionsgruppe mit einem Mittelwert von $36,1^{\circ} \mathrm{C}$ sogar unter der Temperatur der Kontrollgruppe mit 36,2 ${ }^{\circ} \mathrm{C}$ (Mittelwert) liegt. Die Inzidenz intra- und postoperativer Hypothermie ist in der Kontrollgruppe sogar niedriger als in der Interventionsgruppe jedoch ohne statistische Signifikanz. Des Weiteren zeigt sich auch kein signifikanter Unterschied in der Verweildauer im Aufwachraum zwischen den Gruppen. Dieses Ergebnis ist kongruent mit der vergleichbaren Inzidenz für Hypothermie in beiden Gruppen, da das Auftreten von Hypothermie im Vergleich zu Normothermie bei Patienten zu einer verlängerten Verweildauer im Aufwachraum führen kann (Lenhardt et al. 1997). Allerdings finden sich keine Angaben zur Verweildauer im Aufwachraum in anderen Studien zur Hypothermie nach HNO-chirurgischen Eingriffen. Shivering wurde in beiden Studiengruppen mit Clonidin behandelt, ohne dass sich ein signifikanter Unterschied in der Anwendungshäufigkeit zeigt. Diese Ergebnisse entsprechen ebenfalls der vergleichbaren Inzidenz von Hypothermie in beiden Studiengruppen, da gezeigt wurde, dass hypotherme Probanden signifikant häufiger Kältezittern aufweisen (Horn et al. 1998). Allerdings erfolgte die Gabe von Clonidin in dieser Studie nicht nach einem standardisierten Protokoll, sondern gemäß der Einschätzung des Aufwachraumpersonals und ist daher nur mit Einschränkungen zu interpretieren. Zusammenfassend lässt sich daher vermuten, dass die aktive Wärmezufuhr durch die Wärmedecke Barrier ${ }^{\circledR}$ Easywarm ${ }^{\circledR}$ der Isolation durch eine Krankenhaussteppdecke annähernd gleichwertig ist in der Vermeidung perioperativer Hypothermie. Dieses Ergebnis steht im Kontrast zu vielen anderen Studien, die das 
Einhalten höherer Körperkerntemperaturen durch aktive Wärmesysteme im Vergleich zu passiver Isolation demonstrieren (Galvao et al. 2010). Eine mögliche Ursache der fehlenden Überlegenheit des konduktiven Wärmesystems Barrier® Easywarm® gegenüber der Isolation durch eine Krankenhaussteppdecke scheint durch den geringen Anteil aktiver Wärmefläche bedingt zu sein. Diese bemisst sich auf $0,156 \mathrm{~m}^{2}$. Damit tragen lediglich $11 \%$ der Gesamtfläche zum aktiven Wärmetransport bei. Die restlichen 89 \% der Deckenfläche können Wärmeverluste nur durch Isolation der Körperoberfläche verhindern. Obwohl keine Studien zu dem Isolationswert des nicht wärmenden Deckenmaterials vorliegen, scheint dieser am ehesten mit einer chirurgischen Abdeckung vergleichbar zu sein. Diese weisen Isolationswerte von 0,17 bis 0,73 clo auf, die deutlich unter denen einer Krankenhaussteppdecke mit 1,29 clo liegen (Brauer et al. 2004c). Gemäß der Wärmeflussgleichung trägt die wärmende Fläche entscheidend zum Wärmeaustausch bei (English 2001). Weist ein Material zwar in $11 \%$ der Fläche eine aktive Wärmezufuhr, aber in $89 \%$ eine schlechtere Isolation verglichen mit der alleinigen Isolation durch eine Krankenhaussteppdecke auf, ist das eine plausible Erklärung der fehlenden Effektivität des aktiven Wärmesystems.

\subsubsection{Erklärung der Ergebnisse durch die Modelluntersuchung}

Die Testmessungen der Temperaturverläufe der vier Decken zeigten, dass die Decken sehr unterschiedliche Maximaltemperaturen zwischen 36,4 und $42{ }^{\circ} \mathrm{C}$ erreichen. Aus diesen exemplarischen Messungen muss geschlossen werden, dass bei einem Teil der gewärmten Patienten aufgrund der geringen erreichten Deckentemperaturen kein oder nur ein geringer Wärmetransfer möglich war. Da der Wärmefluss von der Temperaturdifferenz zwischen zwei Oberflächen, in dem Fall Decke und Patient, abhängig ist (English 2001), können maximale Deckentemperaturen von $36,4{ }^{\circ} \mathrm{C}$ möglicherweise keinen ausreichenden Wärmefluss zum Patienten zum Einhalten von Normothermie erzeugen.

Allerdings zeigen die Modelluntersuchungen auch, dass die Aktivierungszeit von 30 Minuten ausreichend ist, da die Decken nach dieser Zeit Temperaturen zwischen 35,8 und $38,2^{\circ} \mathrm{C}$ erreichten und im Verlauf bei zwei Decken nur eine Differenz von 0,4 bis $0,6^{\circ} \mathrm{C}$ bis zur maximalen Temperatur innerhalb des vierstündigen Beobachtungsintervalls auftrat. Bei zwei Decken stieg die Temperatur dagegen noch um 2,2 bis $2,4^{\circ} \mathrm{C}$ weiter an und erreichte damit bei einer Decke eine Maximaltemperatur von $42{ }^{\circ} \mathrm{C}$. Dies ist insofern bedenklich, da Sicherheitsnormen für aktive Wärmesysteme einen Grenzwert von $40^{\circ} \mathrm{C}$ festlegen (International Electrotechnical Commission 2009) um das Risiko für Verbrennungen zu minimieren. 
Bei dem Wärmesystem Barrier ${ }^{\circledR}$ Easywarm ${ }^{\circledR}$ ist die Temperatur nicht regelbar, sodass bei diesem Design eine automatische Begrenzung der Maximaltemperatur integriert sein muss. Dies kann dazu führen, dass bei den Decken Temperaturen gemessen werden, welche zum Teil nicht ausreichend effektiv zum Erhalt der Normothermie sind.

Auf der anderen Seite scheint die Begrenzung nicht zuverlässig zu funktionieren, da zum Teil das empfohlene Sicherheitslimit der maximalen Temperatur überschritten wird. Elektrisch regelbare Wärmesysteme haben hier Vorteile und können die Maximaltemperatur deutlich besser begrenzen um die angestrebte Wärmezufuhr sicher zu stellen. Allerdings zeigt sich in einer Studie an einer elektrisch regelbaren konduktiven Wärmematte, dass auch hier in $20 \%$ der Fälle die angewählte Temperatur nicht erreicht wurde (Perl et al. 2012).

\subsection{Kritische Würdigung der Ergebnisse}

\subsubsection{Diskussion der Wärmedecke Barrier® Easywarm® im Vergleich zu anderen konduktiven Wärmedecken}

Das Wärmesystem Barrier ${ }^{\circledR}$ Easywarm $\AA$ vermittelt den Wärmetransfer mittels Konduktion zwischen den Wärmepads und der Körperoberfläche. Dabei sind die Wärmepads so positioniert, dass sich diese über dem Oberkörper und den Extremitäten befinden. Diese Position ist von Vorteil, da ein Großteil der Wärmeverluste über die Körperoberfläche entsteht (Sessler 1997), wohingegen die konduktiven Wärmeverluste über den Rücken zu vernachlässigen sind (Brauer et al. 2006). Daher kann die Wärmebilanz durch über den Rücken zugeführte konduktive Wärmemaßnahmen nur in geringem Maße positiv beeinflusst werden. Dagegen haben sich konduktive Wärmesysteme als effizient erwiesen, die über die Körperoberfläche (Sessler und Moayeri 1990) oder in Form eines Wassermattenanzuges (Taguchi et al. 2004) Wärme übertragen, da sie große Wärmeverluste über diesen Körperregionen reduzieren und dadurch die Wärmebilanz stärker positiv beeinflussen können.

Neben der Position der Wärmedecke hat auch die Größe der gewärmten Körperoberfläche gemäß der Wärmeflussgleichung entscheidend Einfluss auf den Wärmetransfer (English 2001). Berechnet man die Gesamtgröße der wärmenden Fläche der Wärmedecke Barrier ${ }^{\circledR}$ Easywarm ${ }^{\circledR}$ so beträgt diese lediglich $0,156 \mathrm{~m}^{2}$. Somit vermitteln nur $11 \%$ der Fläche der Gesamtgröße eine aktive Wärmezufuhr. Dagegen weist eine auf den Körper gelegte Wärmedecke mit einer Größe von beispielsweise $0,78 \mathrm{~m}^{2}$ eine fünffach größere aktiv wärmende Fläche auf (Sessler und Moayeri 1990). Zu beachten ist, dass der Wärmetransfer 
bei konduktiven Wärmeverfahren nicht allein über die Größe der Wärmematte sondern über die Größe der direkten Kontakt- und Auflagefläche erfolgt (Brauer et al. 2006). So wird bei dem Einsatz von unter den Rücken gelegten Wärmematten nur circa $20 \%$ (Brauer et al. 2004b) und bei dem Einsatz von angepassten Wasseranzügen bis zu 77 \% der Körperoberfläche gewärmt (Taguchi et al. 2004). Da die Wärmepads des Wärmesystems Barrier® Easywarm® sich nicht besonders ergonomisch an den Körper anlegen, kann angenommen werden, dass nicht die komplette Fläche der Wärmepads dem Körper direkt aufliegt. Allerdings würden nur circa 5 bis $10 \%$ der Körperoberfläche aktiv gewärmt werden, selbst wenn der konduktive Wärmetransfer über die ganze Wärmefläche von $0,156 \mathrm{~m}^{2}$ erfolgt. Weiterführende Untersuchungen des Wärmetransfers der Wärmepads - z. B. durch Wärmeflussmessungen am Modell - könnten zusätzliche Erkenntnisse zur Beeinflussung der Wärmbilanz durch das Wärmesystem Barrier® Easywarm® erbringen.

Im Vergleich zu den anderen konduktiven Wärmeverfahren wie Wassermatten und Wassermattenanzügen oder elektrischen Wärmematten, die alle eine Regulation der Temperatur ermöglichen, besteht diese Option der Temperatureinstellung bei dem Wärmesystem Barrier® Easywarm® nicht. Dies ist von Nachteil, da dadurch keine individuelle Wärmezufuhr für den Patienten möglich ist.

Zusammenfassend betrachtet stellt der Einsatz konduktiver Wärmemaßnahmen ein im Mechanismus des Wärmetransfers gut untersuchtes Verfahren dar. Der Wärmetransfer per Konduktion ist von einem wenig effektiven zu einem effizienten Verfahren weiterentwickelt worden. Vergleicht man die Effektivität unterschiedlicher konduktiver Wärmemodelle mit dem Verfahren der konvektiven Luftwärmung, so sind die auf den Körper gelegten Wassermatten ähnlich effektiv. Werden dagegen unter den Rücken gelegte Wassermatten verwendet, sind diese der konvektiven Luftwärmung unterlegen. Dagegen sind als Wassermattenanzüge verwendete konduktive Wärmesysteme sogar suffizienter in der Wärmeübertragung als konvektive Luftwärmer. Somit kann postuliert werden, dass nicht das Verfahren der konduktiven Wärmeübertragung selber, sondern deren Anwendungsdesign die Effektivität der Verhinderung perioperativer Hypothermie definiert. 


\subsubsection{Diskussion der Wärmedecke Barrier® Easywarm® im Vergleich zu konvektiver Luftwärmung}

Beim Einsatz konvektiver Luftwärmung erfolgt der Wärmetransfer anders als bei dem konduktiven Wärmesystem Barrier® Easywarm® über Konvektion. Dabei ist kein enger Kontakt zwischen der Wärmefläche und dem Körper erforderlich (Brauer et al. 2006, English 2001). In vielen Studien ist der Einsatz konvektiver Luftwärmer ausreichend effektiv, um die Körperkerntemperatur in einem normothermen Bereich stabil zu halten oder hypotherme Patienten wieder zu erwärmen (de Brito Poveda et al. 2013). Allerdings ist der alleinige Einsatz konvektiver Wärmemaßnahmen nicht immer ausreichend um Normothermie herzustellen (Leben und Tryba 1997, Smith et al. 1998). Um durch konvektive Wärmemaßnahmen einen maximalen Wärmetransfer zu ermöglichen, müssen ähnliche Voraussetzungen wie bei konduktiven Wärmesystemen erfüllt werden. So ist der Wärmetransfer von der Temperaturdifferenz zwischen Wärmefläche und Haut, der Größe der gewärmten Körperoberfläche sowie deren Einfluss auf die Wärmebilanz abhängig. Daher können mit einer Ganzkörperwärmedecke bis zu 64 \% der Körperoberfläche gewärmt werden (Taguchi et al. 2004). Dies entspricht einer 10- bis 20-fach größeren Wärmefläche als bei dem konduktiven Wärmesystem Barrier® Easywarm®. Der Einfluss der gewärmten Fläche auf die Änderung der Wärmebilanz zeigt sich auch bei dem Einsatz konvektiver Luftwärmer. Obwohl Unterkörperwärmedecken im Vergleich zu Oberkörperwärmedecken einen etwas geringeren Wärmetransfer bewirken, sind sie dennoch effektiver in der Prävention perioperativer Hypothermie, da sie eine größere Fläche bedecken $\left(0,54 \mathrm{~m}^{2}\right.$ versus $\left.0,35 \mathrm{~m}^{2}\right)$. Hierdurch werden die Wärmeverluste durch Radiation und Konvektion über eine größere Fläche besser reduziert, wodurch eine stärkere positive Wärmebilanz resultiert (Brauer et al. 2003). Wird ein größerer Teil der Körpers gewärmt, wie z. B. mit fast 78 \% der Körperoberfläche durch den Einsatz eines konduktiven Wassermattenanzuges (Taguchi et al. 2004), resultiert eine höhere Körperkerntemperatur und damit ein geringere Inzidenz perioperativer Hypothermie.

Bei den konvektiven Luftwärmern besteht im Gegensatz zu dem Wärmesystem Barrier® Easywarm ${ }^{\circledR}$ die Möglichkeit der Temperaturregulation. Dies stellt einen zusätzlichen Sicherheitsaspekt dar, um das Risiko für Verbrennungen durch die Möglichkeit der Temperaturanpassung zu minimieren, da Verbrennungen bei einigen Patienten auch bei niedrigeren Temperaturen auftreten können (Crino und Nagel 1968). 
Des Weiteren steigert die variable und individuelle Einstellung der Wärmezufuhr die Patientenzufriedenheit und Akzeptanz. So zeigt sich in Studien zur Vorwärmung über konvektive Luftwärmer, dass die Patienten eine Reduktion der Wärmezufuhr ausgehend von der Maximaltemperatur gegenüber einer stufenweisen Steigerung der Temperatur bevorzugen und dadurch längere Vorwärmzeiten bei höheren Temperaturen tolerieren (Cobbe et al. 2012).

\subsubsection{Diskussion der Wärmedecke Barrier® Easywarm® im Vergleich zu verschiedenen Isolationsmaterialien}

Da mit 89 \% der Fläche ein Großteil der Wärmedecke Barrier® Easywarm® nicht aktiv wärmt, sondern lediglich den Wärmeverlust per Isolation verhindert, muss man die Isolationseigenschaften mit denen anderer Materialien vergleichen. In früheren Studien zu verschiedenen Isolationsmaterialien stellte sich heraus, dass sich diese kaum in ihren Isolationseigenschaften unterscheiden und in vergleichbarer Größenordnung den Wärmefluss an die Umgebung begrenzen (Sessler et al. 1991a). In einer aktuelleren Studie wurden neben den im Operationssaal üblichen Isolationsmaterialien noch weitere Patientenbedeckungen, wie die in der Kontrollgruppe verwendete Bettdecke, untersucht (Brauer et al. 2004c). Entgegen den Untersuchungen von Sessler et al. (1991a) zeigen Brauer et al. (2007), dass sich andere Isolationsmaterialien wie $z$. B. eine Bettdecke mit 1,29 clo deutlich im Isolationswert von den kommerziell erwerblichen Materialien unterscheiden. Die Isolationswerte dieser Materialien reichen von 0,17 clo bei einer zweilagigen chirurgischen Abdeckung bis zu 0,73 clo bei dem zweilagigen Einsatz einer speziellen Isolationsdecke wie Barkey Thermcare $1 \circledast$ (Brauer et al. 2004c). Diese vermarktete Variante reduziert den Wärmeverlust mit $45 \%$ am stärksten (Brauer et al. 2004c) verglichen mit vielen anderen kommerziellen Isolationsmaterialien, bei denen die Reduktion bei circa 30 \% liegt (Sessler et al. 1991a). Werden dagegen doppelt gelegte Steppdecken mit einem resultierenden Isolationswert von 2,79 clo verwendet, lässt sich der Wärmeverlust bis zu $80 \%$ reduzieren (Brauer et al. 2004c).

Die Wärmedecke Barrier ${ }^{\circledR}$ Easywarm $\AA$ besteht aus Polypropylen, welches auch in Operationsabdeckungen an der Patientenseite Verwendung findet. Daher ist anzunehmen, dass der Isolationswert dieser Decke in einem Bereich von chirurgischen Operationsabdeckungen oder Bettlaken anzusiedeln ist, obwohl diesbezüglich keine Daten vorliegen.

Um die Effektivität der Wärmedecke Barrier ${ }^{\circledR}$ Easywarm ${ }^{\circledR}$ zu verbessern, wäre es wünschenswert, die nicht aktiv wärmende Fläche mit einem besser isolierenden Material auszustatten. Da weiterhin der Wärmetransfer von den Wärmepads 
zu der Körperoberfläche des Patienten ermöglicht werden soll, muss diese aktiv wärmende Fläche möglichst geringe Isolationseigenschaften aufweisen.

Als theoretische Möglichkeit besteht die Ausstattung der Wärmedecke Barrier® Easywarm ${ }^{\circledR}$ mit einem reflektierenden Material um die radiativen Wärmeverluste besser zu reduzieren. So zeigen Brauer et al. (2007) am Modell, dass der Einsatz eines reflektierenden Isolationsmaterials (z. B. Thermadrape) effektiver als der Einsatz chirurgischer Abdeckungen in der Vermeidung von Wärmeverlusten ist (Brauer et al. 2007). Demgegenüber kann allerdings in vielen klinischen Studien keine Überlegenheit eines reflektierenden Materials alleine (Bennett et al. 1994, Berti et al. 1997) oder als zusätzliche Maßnahme (Brauer et al. 2000) in der Reduktion perioperativer Hypothermie demonstriert werden. Mögliche Ursachen für eine fehlende Effektivität reflektierender Materialien können durch Einschränkungen im praktischen Einsatz bedingt sein. So kann beispielsweise der nötige Abstand zwischen Haut und Radiator in der Praxis aufgrund von Abdeckungen und zusätzlichem Druck von oben auf den Radiator im chirurgischen Umfeld nicht immer eingehalten werden (Brauer et al. 2004c).

Eine dritte theoretische Option, die Wärmeverluste durch verbesserte Isolationseigenschaften zu reduzieren, bestände darin den Patienten mit einem besser isolierenden Material wie einer Krankenhaussteppdecke zusätzlich zu bedecken. Hiergegen spricht allerdings, dass sich durch den zusätzlich entstehenden Druck die Gefahr für Hautläsionen durch Verbrennungen erhöhen kann. Zudem ist laut Hersteller eine Druckbelastung auf den Wärmepads zu vermeiden, sowie der Einsatz einer durch Faltung doppelt gelegten Wärmedecke untersagt (Gebrauchsanweisung Barrier ${ }^{\circledR}$ Easywarm ${ }^{\circledR}$, Mönlycke Healthcare $\mathrm{GmbH}$, Erkrath, Deutschland 2012, Seitenabruf am 01.09.2014, siehe Anhang).

\subsubsection{Diskussion von Wirtschaftlichkeit und Patientenkomfort der Wärmedecke Barrier® Easywarm ${ }^{\circledR}$}

Neben einer Verbesserung der Effektivität der Isolationseigenschaften scheinen auch die Materialeigenschaften entscheidend für die Akzeptanz der Decke unter dem Aspekt des Patientenkomforts zu sein. In der Interventionsgruppe verlangten postoperativ $55 \%$ der Patienten nach einer normalen Bettdecke bzw. einer anderen Wärmemaßnahme. Betrachtet man den postoperativen Einsatz konvektiver Luftwärmer, so wurde dieser bei $17 \%$ der Patienten der Interventionsgruppe und bei $9 \%$ der Patienten der Kontrollgruppe verwendet. Letztlich ließen $38 \%$ der Patienten der Interventionsgruppe die Wärmedecke Barrier ${ }^{\circledR}$ Easywarm ${ }^{\circledR}$ gegen eine normale Bettdecke im Aufwachraum austauschen. Daher ist anzunehmen, dass die Wärmedecke Barrier ${ }^{\circledR}$ Easywarm $\AA$ im empfundenen Komfort 
gegenüber einer normalen Krankenhaussteppdecke unterlegen ist und im Hinblick auf die Materialeigenschaften verbessert werden könnte.

Der Einkaufspreis der Decke für diese Studie beträgt 11,40€ pro Stück bei einer Abnahme von 60 Stück. An der Universitätsmedizin Göttingen werden konvektive Luftwärmer als Standardwärmemaßnahme mit Ganzkörper- oder Unterlegdecken eingesetzt. Die Verwendung dieser Decken kostet dort pro Gebrauch bei einer Unterlegdecke Typ-2 $7 €$ und bei einer Ganzkörperdecke 3,50 €. Damit liegen die Kosten für den Einsatz des neuen konduktiven Wärmesystems über denen der bisher dort üblichen Wärmemaßnahmen.

\subsection{Limitationen der Studie}

Kritisch diskutiert werden müssen die Limitationen, die durch das Studiendesign bedingt sind und die Interpretation der Ergebnisse einschränken.

Die Ergebnisse basieren grundlegend auf den Temperaturmessungen der Körperkerntemperatur, die mit unter $36^{\circ} \mathrm{C}$ als Definition für Hypothermie gemäß den NICE-Guidelines herangezogen wird (NICE 2008). Mögliche Messorte der Körperkerntemperatur sind z. B. in der Pulmonalarterie, am Trommelfell, im distalen Ösophagus und in der Blase (Lefrant et al. 2003, Torossian 2008).

Den Goldstandard der Bestimmung der Körperkerntemperatur stellt die Temperaturmessung in der Pulmonalarterie dar (Torossian 2008). Dieses Verfahren ist allerdings bei nicht anästhesierten Patienten und kleinen Operationen unter dem Aspekt der Nutzen-Risiko-Relation praktisch nicht anwendbar.

Die Kontakttemperaturmessung am Trommelfell wird trotz einiger Schwierigkeiten in der präzisen Anwendung und gewisser Invasivität aufgrund der erforderlichen Nähe zur Membrana tympani in vielen Studien angewendet und gilt als vergleichbar mit dem Goldstandard der pulmonalarteriellen Temperaturmessung (Torossian 2008). Diese Messmethode ist jedoch insbesondere bei Eingriffen im Kopfbereich problematisch, da hierbei das Risiko einer Trommelfellperforation erhöht ist. Zudem entstehen bei HNO-chirurgischen Eingriffen, z. B. bei beidseitigen Ohroperationen, Interferenzen mit dem Operationsbereich.

Die Genauigkeit und Präzision der distalen Ösophagustemperatur ist zur Erfassung der Körperkerntemperatur klinisch hinreichend genau und vergleichbar der Kontakttemperaturmessung am Trommelfell (Patel et al. 1996).

Die Messung der Blasentemperatur steht bei fehlender Notwendigkeit eines Blasenkatheters in der Praxis als Messort nicht zur Verfügung und ist bezüglich der 
Genauigkeit der Temperaturmessung von der Urinproduktion abhängig (Sato et al. 2008).

Eine aktuelle Untersuchung hat gezeigt, dass die Messung sublingualer Temperaturen sowohl prä- als auch intraoperativ ausreichend genaue und präzise Messergebnisse der Körperkerntemperatur im Vergleich mit der Kontakttemperaturmessung am Trommelfell liefert. Die Unterschiede zwischen beiden Verfahren liefern eine um 0,1 bis $0,2^{\circ} \mathrm{C}$ höhere Temperatur über die sublinguale Messmethode, sodass diese Methode bezüglich Validität und Genauigkeit für die gesamte perioperative klinische Praxis empfohlen werden kann (Hocker et al. 2012).

Als Limitation in dieser Studie ist der Wechsel der Messorte anzusehen. Die präoperative Temperatur wurde über die sublinguale Messung unter der Zunge in der hinteren Tasche durchgeführt, dagegen erfolgte intraoperativ die Temperaturmessung im distalen Ösophagus. Obwohl beide Methoden hinreichend genau zur Bestimmung der Körperkerntemperatur sind, wird empfohlen, die Temperatur perioperativ nur an einem Ort zu messen (Hooper et al. 2009). Da die sublinguale Temperaturmessung aufgrund der intraoperativen Gegebenheiten im HNO-Operationssaal schwierig zu realisieren ist und andere hinreichend genaue Messmethoden wie z. B. die Temperaturmessung im distalen Ösophagus präoperativ bei wachen Patienten zu invasiv sind, ist in dieser Studie ein Wechsel der Messmethoden nicht zu vermeiden. Allerdings resultiert durch diesen Wechsel ein systematischer Fehler, der auch bei Betrachtung der Temperaturen nach Narkoseeinleitung deutlich wird. Hier liegen die ösophageal gemessenen Temperaturen um 0,3 bis $0,4{ }^{\circ} \mathrm{C}$ höher als die präoperativ sublingual gemessenen Temperaturen. Zu erwarten gewesen wäre dagegen ein durch die Blutumverteilung bedingter Abfall der Körperkerntemperatur nach der Narkoseeinleitung. Um den Einfluss des Wechsels der Messorte abzuschwächen, wurde bereits fünf Minuten nach der Narkoseeinleitung die Ösophagustemperatur bestimmt. Betrachtet man diese Messung als Ausgangswert, so ergibt sich bis zur niedrigsten gemessenen Körperkerntemperatur ein maximaler Abfall um $0,5^{\circ} \mathrm{C}$. Dieser Wert ist zwar deutlich kleiner als der in der Erstdokumentation von Matsukawa et al. (1995b) beschriebene Temperaturabfall von 1,6 ${ }^{\circ} \mathrm{C}$ nach Narkoseeinleitung, aber in seiner Größenordnung vergleichbar mit den Daten einer Studie von Perl et al. (2012) an einem ähnlichen Patientenkollektiv. Daraus lässt sich schließen, dass die Patienten beider Gruppen eine vergleichsweise gute Wärmeprotektion während des Transportes in den Operationssaal und vor der Narkoseeinleitung erhalten haben, sodass die peripheren Gewebe nicht zu stark ausgekühlt sind, 
wodurch ein kleinerer Abfall der Körperkerntemperatur nach Narkoseeinleitung resultieren kann.

Aus dieser Studie mussten zwölf Patienten (15\%) aufgrund von Fehlein-schätzungen der Operationszeit ausgeschlossen werden, da die tatsächliche Operationsdauer außerhalb der Zeitspanne von 60 bis 180 Minuten lag. Dieses Problem ist nicht ungewöhnlich für Studien, die eine zeitliche Limitierung im Studiendesgin beinhalten. So mussten in einer Studie von Perl et al. (2012) mit vergleichbarem Studiendesign und vergleichbarer Patientengruppe $25 \%$ der Studienteilnehmer aufgrund der Operationszeiten ausgeschlossen werden. Eine zeitliche Einschränkung der Operationsdauer erscheint sinnvoll, da bei zu kurzer Wärmedauer aufgrund der schnellen Umverteilung in der ersten Phase nach Einleitung der Allgemeinanästhesie die Patienten hypotherm wären, ohne dass die Wärmemaßnahmen in ihrer Effektivität ausreichend beurteilt werden könnten (Matsukawa et al. 1995b).

Die Überlegung, dass die Aktivierungszeit der Decke von 30 Minuten zur Entwicklung des angestrebten Temperaturniveaus nicht ausreichend ist, kann anhand der Modellmessungen an vier Decken nicht bestätigt werden. Des Weiteren stand mit einer mittleren Vorwärmzeit von 85 Minuten in der Interventionsgruppe ausreichend Zeit zur vollständigen Aktivierung der Decken sowie Vorwärmung der Patienten zur Verfügung. Zudem haben aktuelle Studien gezeigt, dass bereits Vorwärmzeiten von zehn Minuten ausreichen um das Auftreten von Hypothermie signifikant zu reduzieren (Horn et al. 2012). Grundvorrausetzung hierfür ist allerdings, dass ein Wärmeverfahren gewählt wird, mit dem eine effektive Wärmeübertragung möglich ist.

Die Frage nach der Akzeptanz des Wärmeverfahrens sowie die Behandlung des Kältezitterns durch Clonidin stellten in dieser Studie keine Arbeitshypothesen dar, sondern wurden zusätzlich ausgewertet. Daher wäre es wünschenswert gewesen, die Patientenzufriedenheit über einen standardisierten Fragebogen bezüglich des durch die Decke vermittelten prä- und postoperativen Wärmegefühls und Komfortempfindens zu ermitteln. Kritisch anzumerken ist, dass der Einsatz von Clonidin sowie aktiver Wärmetherapie gemäß dem klinik-üblichen Vorgehen im Aufwachraum erfolgte, ohne dass für diese Studie eine standardisierte Therapieanweisung hinterlegt war. Des Weiteren lag keine Protokollierung der Einstufungen des Grades des Kältezitterns vor, wie es in klinischen Studien deren primäre Fragestellungen den Themenbereich Shivering umfassen, häufig verwendet wird (Horn et al. 1999). Daher sind die Ergebnisse der postoperativen 
Therapie shivernder und hypothermer Patienten nur mit Einschränkungen zu interpretieren. In folgenden Studien sollte für die postoperative Therapie ebenfalls ein standardisiertes Studienprotokoll erstellt werden. 


\section{Zusammenfassung}

Perioperative Hypothermie, definiert durch einen Abfall der Körperkerntemperatur unter $36^{\circ} \mathrm{C}$, ist eine der häufigsten Nebenwirkungen der Allgemeinanästhesie (NICE 2008). Das Auftreten perioperativer Hypothermie ist mit einer Vielzahl von Komplikationen wie der Beeinträchtigung von Gerinnung (Rajagopalan et al. 2008), Zunahme von Wundinfektionen (Kurz et al. 1996) und tödlichen kardiovaskulären Ereignissen assoziiert (Frank et al. 1997a). Des Weiteren erhöhen sich die wirtschaftlichen Kosten bedingt durch die mit der Behandlung der Hypothermie assoziierten Komplikationen (Bock et al. 1998) sowie durch die Verlängerung der Aufwachraum- und Krankenhausaufenthaltsdauer (Kurz et al. 1996, Lenhardt et al. 1997). Damit führt perioperative Hypothermie zu einer Erhöhung von Morbidität, Mortalität und ökonomischen Kosten.

Zur Prävention perioperativer Hypothermie stehen eine Vielzahl von Wärmemaßnahmen und Wärmesystemen zur Verfügung. Studien zu unterschiedlichen Wärmeverfahren haben ergeben, dass sich diese in der Effektivität der Verhinderung perioperativer Hypothermie sehr unterscheiden (Galvao et al. 2009). Wird bei den Wärmesystemen die Möglichkeit des Vorwärmens vor Narkoseeinleitung genutzt (sogenanntes Prewarming), wird dadurch das Auftreten von Hypothermie effektiv reduziert (de Brito Poveda et al. 2013). Das neue konduktive Wärmesystem Barrier ${ }^{\circledR}$ Easywarm ${ }^{\circledR}$ enthält Wärmepads, die über eine exogene chemische Reaktion Wärme erzeugen und keinen Strom benötigen. Damit bietet das System die Möglichkeit, den Patienten in der gesamten perioperativen Phase zu wärmen.

Diese Arbeit beschäftigt sich mit der Fragestellung, ob durch den Einsatz des neuen konduktiven Wärmesystems Barrier® Easywarm® das Auftreten perioperativer Hypothermie signifikant reduziert wird. Des Weiteren wird untersucht, ob HNO-Patienten von einem relevanten Risiko für perioperative Hypothermie betroffen sind.

In die Studie wurden 80 Patienten, die sich einem HNO-chirurgischen Eingriff mit einer Dauer von 60 bis 180 Minuten unterziehen, eingeschlossen und in zwei gleich große Gruppen randomisiert. Die Patienten der Kontrollgruppe erhielten eine normale Krankenhaussteppdecke und keine aktiven Wärmemaßnahmen. Die Patienten der Interventionsgruppe wurden mit dem Wärmesystem Barrier® Easywarm® mindestens 30 Minuten vor Narkoseeinleitung vorgewärmt und behielten diese Decke während der Operation. Präoperativ erfolgte die Temperaturmessung sublingual, intraoperativ ösophageal. Ein Temperaturabfall unter 
$36^{\circ} \mathrm{C}$ wurde als Hypothermie definiert. In die statistische Analyse konnten 29 Patienten der Interventionsgruppe und 32 Patienten der Kontrollgruppe eingeschlossen werden.

In der Kontrollgruppe waren $31 \%$ der Patienten und in der Interventionsgruppe sogar $41 \%$ der Patienten intraoperativ hypotherm. Damit wurde durch den Einsatz des neuen konduktiven Wärmesystems das Auftreten perioperativer Hypothermie nicht signifikant verhindert $(p=0,44)$.

Insgesamt liegt die Inzidenz perioperativer Hypothermie bei HNO-Patienten in dieser Studie bei $36 \%$ und tritt daher auch bei HNO-chirurgischen Eingriffen von mittlerer Dauer in relevantem Maße auf.

Die ermittelten Hypothermienzidenzen sind in der Größenordnung vergleichbar mit den Ergebnissen der wenigen, bisherigen Studien bei HNO-Eingriffen (Perl et al. 2012). Jedoch lässt sich aus den vorliegenden Daten schließen, dass auch HNO-Patienten in relevantem Maße von perioperativer Hypothermie betroffen sind (Agrawal et al. 2003, Sumer et al. 2009). Daher sollten auch bei dieser Patientengruppe adäquate Wärmemaßnahmen durchgeführt werden.

Mögliche Ursachen für die unzureichende Effektivität des Wärmesystems Barrier ${ }^{\circledR}$ Easywarm ${ }^{\circledR}$ können dadurch bedingt sein, dass der aktiv wärmende Anteil der Decke mittels Wärmepads aus lediglich $0,15 \mathrm{~m}^{2}$ besteht. $89 \%$ der Decke verhindern den Wärmeverlust lediglich passiv über Isolation und im Vergleich mit der konventionellen Krankenhaussteppdecke aufgrund der Materialeigenschaften sogar schlechter. Des Weiteren zeigten die Wärmesysteme eine sehr unterschiedliche Temperaturentwicklung der Wärmepads, wobei die vom Hersteller angegebene Deckentemperatur teilweise nicht erreicht oder auch überschritten wurde.

Zusammenfassend lässt sich festhalten, dass Hypothermie auch bei HNOchirurgischen Patienten in relevantem Maße auftritt. Daher sollten auch an diesem Patientenkollektiv Studien zur Prävention perioperativer Hypothermie mit klinisch etablierten Wärmemaßnahmen durchgeführt werden um zu überprüfen, ob hierdurch die Hypothermierate deutlich reduziert werden kann. Ebenfalls müssen neue Wärmesysteme wie in dieser Studie auf ihre Effizienz in der Reduktion perioperativer Hypothermie untersucht werden, damit Patienten eine effektive Prävention oder Therapie erhalten und nicht durch die Folgen unerwünschter Hypothermie gefährdet sind. 


\section{ANHANG}

\subsection{Gebrauchsanweisung Barrier ${ }^{\circledR}$ Easywarm ${ }^{\circledR}$}

\section{BARRIER ${ }^{\circ}$ EasyWarm}

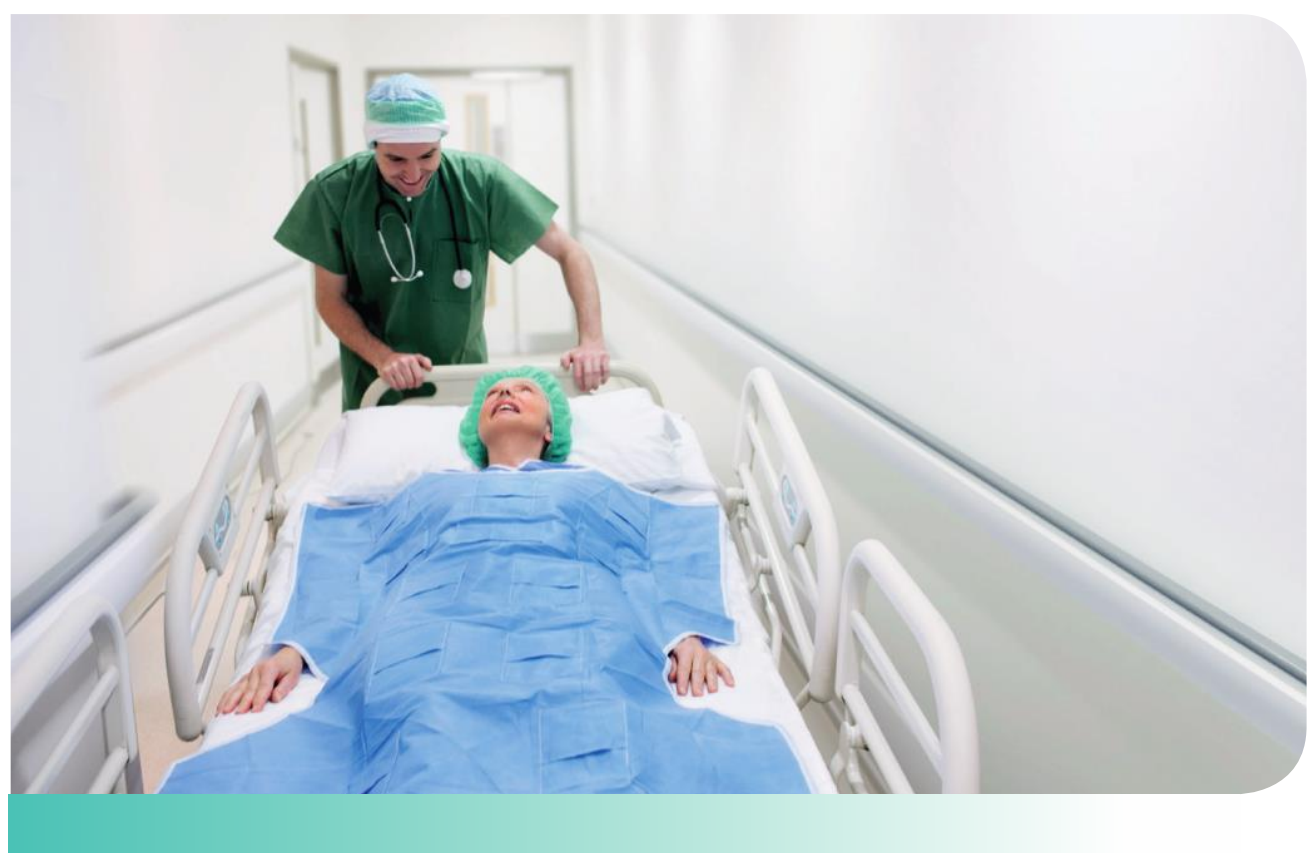

Aktiv selbstwärmende Patientendecke

- Automatische Erwärmung der Decke nach

Öffnung. Die Decke erwärmt sich innerhalb

von 30 Minuten auf durchschnittlich $44^{\circ} \mathrm{C}$ und

hält die Temperatur über einen Zeitraum von

10 Stunden. Die Hauttemperatur unter den

Wärmepads erreicht ein Maximum von $42^{\circ} \mathrm{C}$

- Hilft, eine Hypothermie in der gesamten perioperativen Phase zu verhindern.

- Ohne Zubehör und Strom einsetzbar, daher

sehr flexibel für den prä-, intra- sowie

postoperativen Bereich.

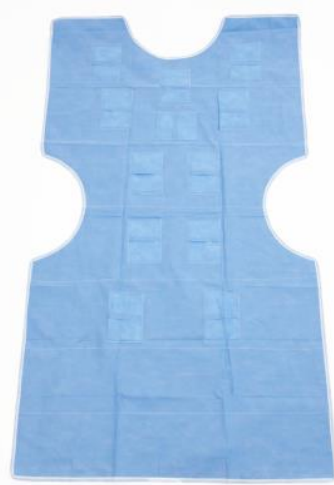


Gebrauchsanweisung

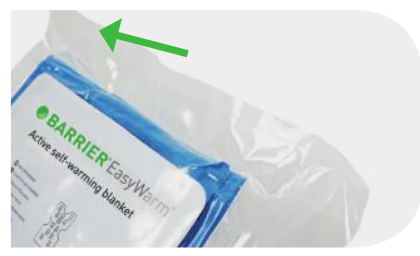

1. Öffnen Sie die Einzelverpackung an der Einkerbung in der oberen linken Ecke. Entnehmen Sie BARRIER ${ }^{\circledR}$ EasyWarm $^{\circledR}$ aus der Umverpackung

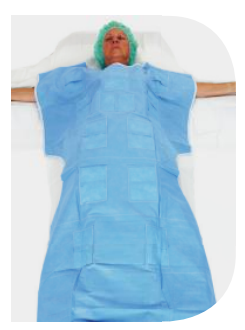

4. Während der prä- und postoperativen Phase sowie während operativer Eingriffe an Hand, Arm oder Kopf breiten Sie BARRIER EasyWarm über dem gesamten Körper aus

Anwendungshinweise

- Die Decke nicht doppelt legen.

- Bei Röntgenuntersuchungen nicht auf den zu röntgenden Bereich legen und keine Anwendung bei MRT.

- Die Wärmepads müssen nach oben zeigen.

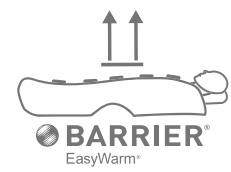

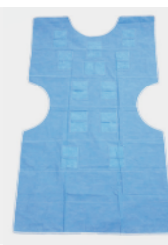

2. Öffnen und entfalten Sie BARRIER EasyWarm vollständig, achten Sie darauf, die Decke nicht doppelt zu legen. Die Patientendecke erwärmt sich innerhalb von 30 Minuten selbständig auf durchschnittlich $44^{\circ} \mathrm{C}$ und hält die Temperatur über einen Zeitraum von etwa 10 Stunden.

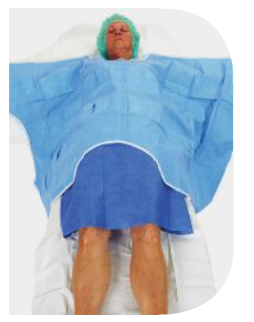

5. Für Eingriffe im unteren abdominalen Bereich und den unteren Extremitäten breiten Sie BARRIER EasyWarm über die oberen Extremitäten sowie dem Brustkorb aus.

- Es wird empfohlen, direkten Kontakt mit Blutergüssen, Schwellungen und Erfrierungen zu vermeiden. Bei Patienten mit verminderter Sensibilität und eingeschränkter Reaktionsfähigkeit
sollte eine mögliche Hautreaktion gemäß sollte eine mögliche Hautreaktion gemäß
der Krankenhausroutinen regelmäßig überprüft werden.

- Nach Gebrauch kann BARRIER

EasyWarm mit dem Hausmüll entsorgt werden. Eine Verbrennung ist möglich.

- Anwendung nur unter ärztlicher oder pflegerischer Aufsicht.

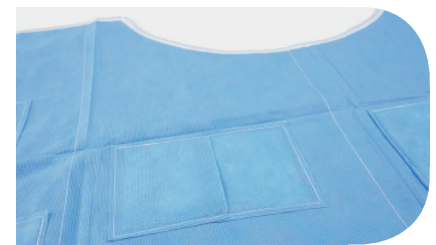

3. Platzieren Sie BARRIER EasyWarm mit den Wärmepads nach oben. Vermeiden Sie zusätzliches Gewicht auf den Wärmepads.

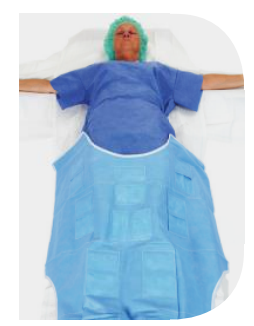

6. Für Eingriffe oberhalb des Genitalbereiches platzieren Sie BARRIER EasyWarm über den unteren Bereich des Körpers

Warnhinweise

- Die Wärmedecke ist ein aktives

therapeutisches Produkt und darf nur entsprechend der Gebrauchsanweisung eingesetzt werden, um mögliche thermische Verletzungen zu vermeiden

- Bei anhaltender Hypotonie sollte der Einsatz der Patientendecke unterbleiben.

- BARRIER EasyWarm ist nicht für die Anwendung bei Personen unter 18 Jahren geeigne

- Die Patientendecke sollte nicht in Fällen von schweren peripheren Gefäßerkrankungen zum Einsatz kommen.

\begin{tabular}{l|l}
\hline Artikel-Nr. & Beschreibung \\
629900 & BARRIER EasyWarm aktiv selbstwärmende Patientendecke \\
& Produkt: 12 luftaktivierte Wärmepads $(13 \mathrm{~cm} \times 10 \mathrm{~cm})$, die in Taschen auf der Oberfläche integriert sind. Die Größe der Decke \\
beträgt $152 \mathrm{~cm} \times 92 \mathrm{~cm}$. \\
Material: Verschiedene Lagen aus SMS (Polypropylen). Die einzelnen Lagen sowie die Taschen sind mit Polyester/ \\
Baumwollgarn miteinander vernäht. Die Wärmepads bestehen aus luftdurchlässigen Beuteln, die Aktivkohle, Mineralien, \\
Salz und Eisenpulver beinhalten. \\
Verpackung: Vakuumverpackt in einem luftdichten Polymerbeutel. Die Beutel sind mit Luftpolsterfolie in einem \\
\\
$\begin{array}{l}\text { Transportkarton eingeschlagen. } \\
\text { Versandeinheit: } 10 \text { Stück }\end{array}$ \\
\hline
\end{tabular}

Besuchen Sie uns auf www.molnlycke.com/de-de/

Mölnlycke Health Care GmbH, Max-Planck-Straße 15, 40699 Erkrath.

Mölnlycke Health Care GmbH, Max-Planck-Straße 15, 40699 Erkrath.
$T+49$ (0) $211920880 . \mathrm{F}+49$ (0) 21192088170 . www.molnlycke.com/de-de/

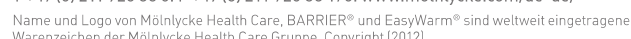

http://www.molnly-

cke.de/Documents/DEU/Surgical/EasyWarm/DE BARRIER EasyWarm Produ ctSheet\%20291113.pdf (Seitenabruf am 01.09.2014) mit freundlicher Genehmigung von Mölnlycke Health Care $\mathrm{GmbH}$ ) 


\section{Anwendungshinweise BARRIER ${ }^{\circ}$ EasyWarm ${ }^{\circ}$ - die aktiv selbstwärmende Patientendecke}

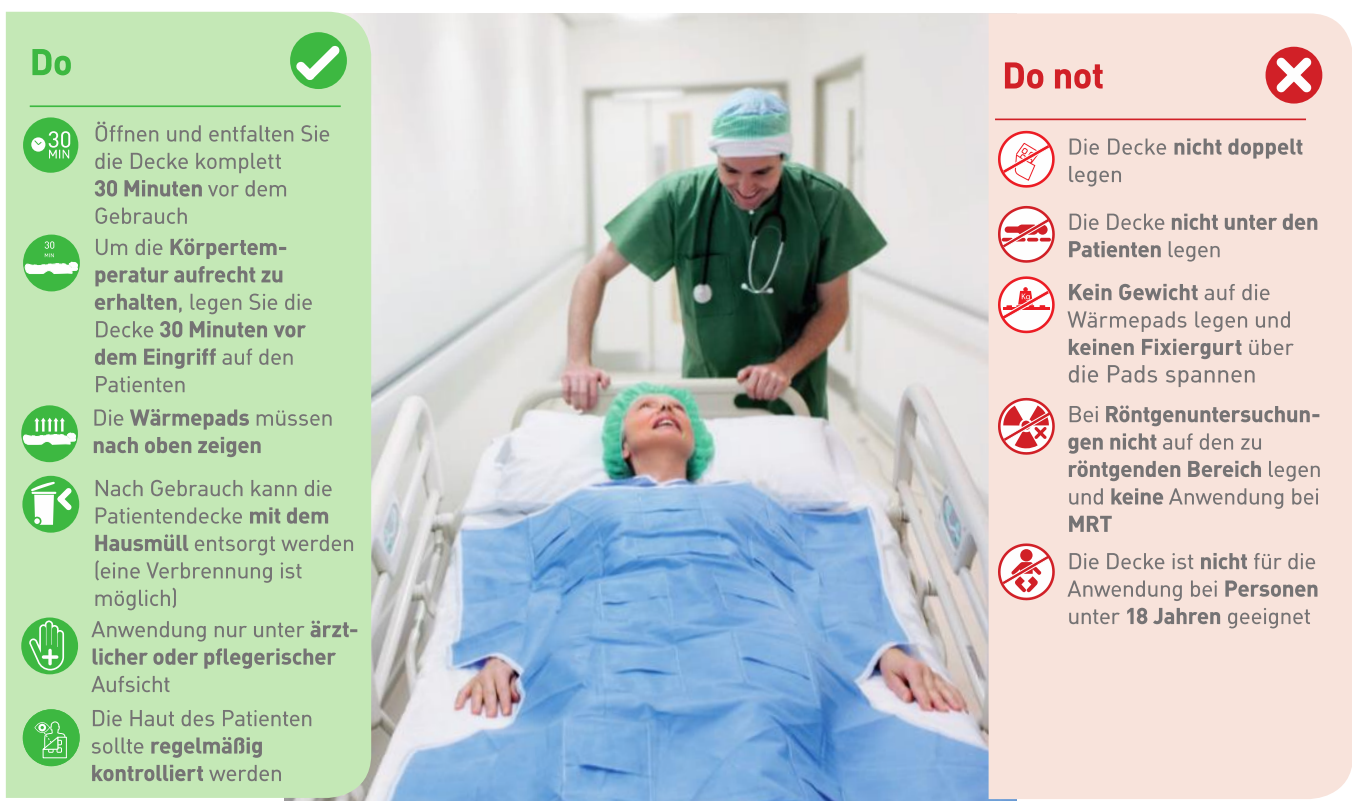

Achtung: Die Patientendecke sollte nicht in Fällen einer beeinträchtigten peripheren Zirkulation (Haut eingeschlossen) zum Einsatz kommen, z.B. bei schwerer oder

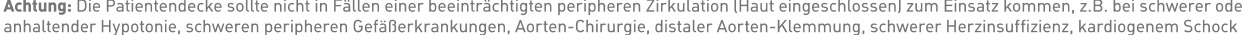

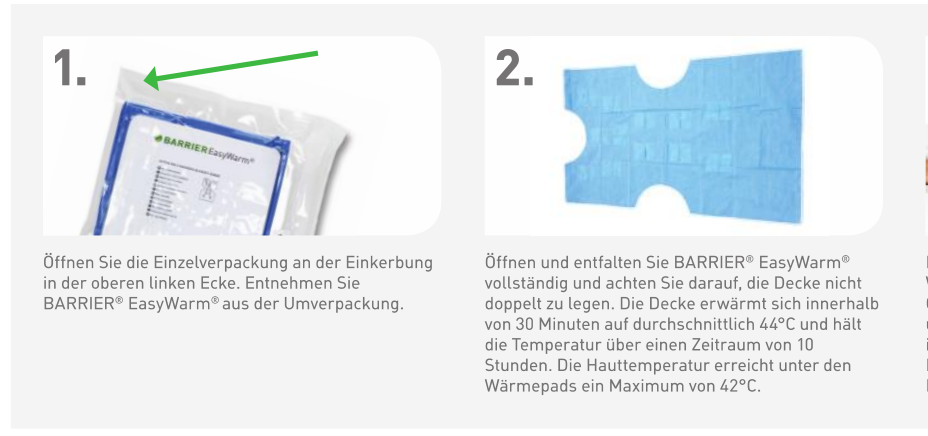

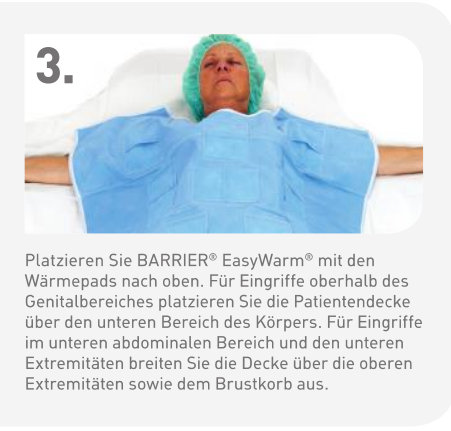

BARRIEREasyWarm ster A3 v1 291113.pdf (Seitenabruf am 01.09.2014) 


\subsection{Messprotokoll}

1. Biometrische und eingriffsspezifische Daten

\begin{tabular}{|l|l|l|}
\hline Patienten-ID & Größe (cm) & ASA \\
\hline Geschlecht & Gewicht $(\mathrm{kg})$ & Art des Eingriffs \\
\hline Alter & BMI $\left(\mathrm{kg} / \mathrm{m}^{2}\right)$ & OP Dauer geplant (min) \\
\hline Gruppe: Vorwärmung $\bigcirc$ & \multicolumn{2}{|c|}{ Kontrolle $\bigcirc$} \\
\hline
\end{tabular}

2. Verbrauch Anästhetika und Infusionen

\begin{tabular}{|l|l|l|}
\hline Ultiva (mg) & Rocuronium (mg) & Kristalloide (ml) \\
\hline Propofol (mg) & & Kolloide (ml) \\
\hline
\end{tabular}

\section{Messwerte Temperaturen}

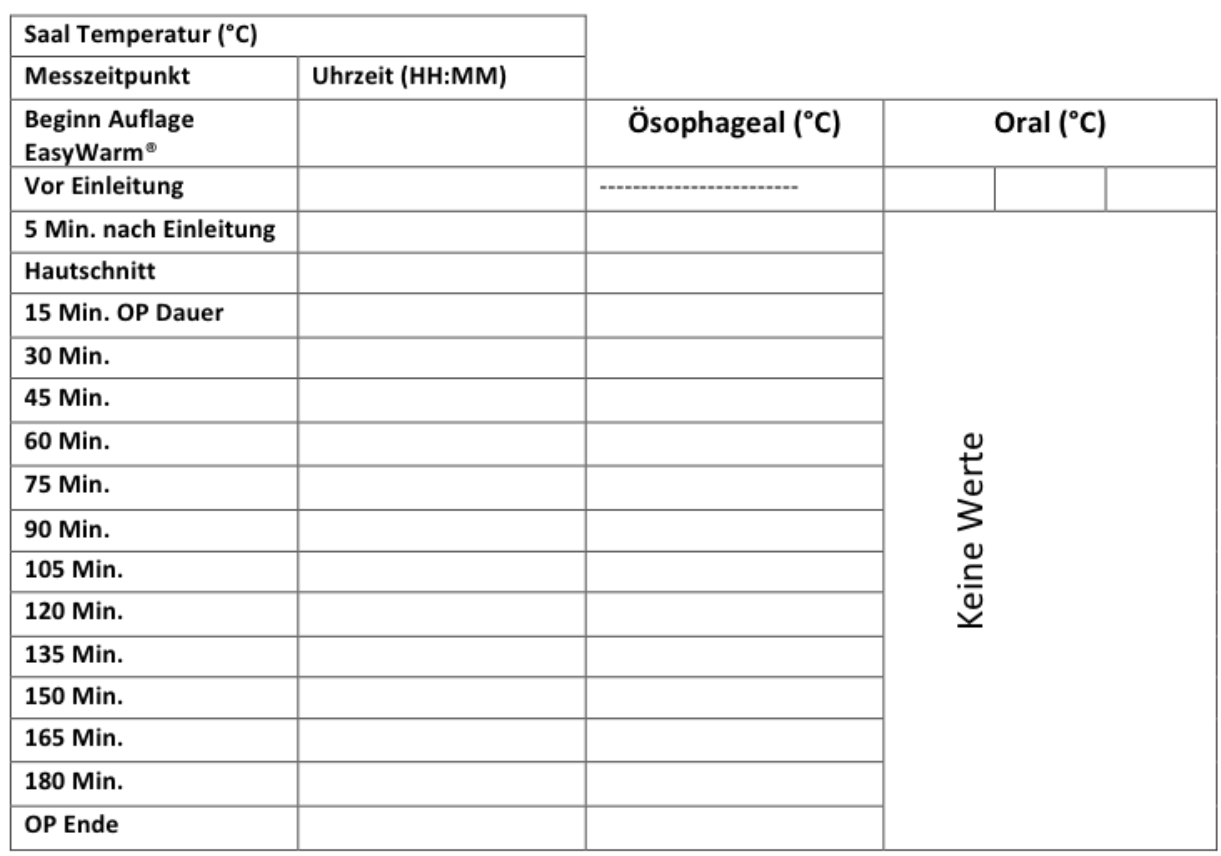

4. Verweildauer Aufwachraum (min):

Ankunft:

Entlassung: 


\title{
6.3 Patientenaufklärung
}

\author{
Dr. med. Ivo Brandes \\ Prof. Dr. med. Anselm Bräuer, DEAA \\ Universitätsmedizin Göttingen \\ Zentrum Anaesthesiologie, Rettungs- und Intensivmedizin \\ Robert-Koch-Str. 40 \\ 37099 Göttingen \\ Telefon +49 551 39-22995
}

Über den gesamten Zeitraum der Operation sowie im Aufwachraum wird Ihre Körpertemperatur in regelmäßigen Abständen gemessen und dokumentiert. Die Temperaturmessung erfolgt vor Operationsbeginn oral, während der Operation über eine Messsonde in der Speiseröhre. Zusätzlich wird die Temperatur vor und während der Operation an der Stirn über einen Aufkleber auf der Haut gemessen.

Darüber hinaus wird Ihre Verweildauer im Aufwachraum dokumentiert. Die erhobenen Daten werden unter einer Codenummer gespeichert, die nicht Ihrer Person zuzuordnen ist (pseudonomysiert). Die Soeicherungsdauer beträgt 10 Jahre. Sie haben jederzeit die Möglichkeit die Teilnahme an der Studie zu Widerrufen und die Möglichkeit die erhobenen Daten löschen zu lassen.

Risiken und Nebenwirkungen dieser Wärmetherapie sind bisher nicht bekannt.

Die Teilnahme an dieser Untersuchung ist freiwillig. Wenn Sie nicht teilnehmen wollen, erhalten Sie die Standardbehandlung. Sollten Sie sich im weiteren Verlauf gegen die Teilnahme an der Studie entscheiden, haben sie jederzeit die Möglichkeit diese Einwilligung zu wiederufen. Evtl. bis dahin gespeicherte pseudonomysierte Daten werden in diesem Fall gelöscht.

\section{Notfalladressen:}

Sollten sich während der Studie unerwünscht Ereignisse ergeben oder Sie noch Fragen, Anregungen oder Wünsche haben erreichen Sie unter nachstehender Adresse jederzeit einen der Prüfärzte.

\section{Dr. med. Ivo Brandes}

Prof. Dr. med. Anselm Bräuer, DEAA

Univer sitätsmedizin Göttingen

Zentrum Anaesthesiologie, Rettungs und Intensivmedizin

Robert-Koch-Str. 40

37099 Göttingen

Telefon +49 551 39-22995

E-Mail abraeue@gwdg.de 


\title{
6.4 Patienteneinwilligung
}

\author{
Dr. med. Ivo Brandes \\ Prof. Dr. med. Anselm Bräuer, DEAA \\ Universitätsmedizin Göttingen \\ Zentrum Anaesthesiologie, Rettungs- und Intensivmedizin \\ Robert-Koch-Str. 40 \\ 37099 Göttingen \\ Telefon +4955139-22230
}

\section{Patienteneinverständniserklärung zur Studie}

\section{„, Untersuchung der Effektivität eines neuen konduktiven Vorwärmesystems zur Prävention perioperativer Hypothermie bei chirurgischen Eingriffen im Bereich der Hals-Nasen-Ohrenheilkunde "}

Patientenaufkleber/Patientencode

\begin{abstract}
Ich ................................, w urde von meinem Arzt über Wesen und Tragweite der klinischen Prüfung mit dem o. g. Titel aufgeklärt. Ich habe den Aufklärungstext gelesen und verstanden. Ich hatte die Möglichkeit, Fragen zu stellen, und habe die Antworten verstanden und akzeptiere sie. Mein Arzt hat mich über die mit der Teilnahme an der Studie verbundenen Risiken und den möglichen Nutzen informiert.

Ich hatte ausreichend Zeit, mich zur Teilnahme an dieser Studie zu entscheiden und weiß, dass die Teilnahme an dieser klinischen Prüfung, die damit verbundene Datenerhebung und die pseudonymisierte Verarbeitung und Speicherung, freiwillig ist. Ich weiß dass ich jederzeit und ohne Angabe von Gründen diese Zustimmung widerrufen kann, ohne dass sich dieser Entschluss nachteilig auf die spätere Behandlung durch meinen Arzt auswirken wird.
\end{abstract}

\section{Mir ist bekannt, dass meine persönlichen Daten in verschlüsselter Form gespeichert werden.}

Ich habe eine Kopie der Patienteninformation und dieser Einwilligungserklärung erhalten. Ich erkläre hiermit meine freiwillige Teilnahme an dieser klinischen Studie. 


\subsection{Randomisierungsliste}

Seite 1 von 2

\section{A Randomization Plan \\ from \\ http://www.randomization.com}

1. EasyWarm

2. Control

3. Control

4. EasyWarm

5. Control

6. EasyWarm

7. Control

8. Control

9. Control

10. EasyWarm

11. EasyWarm

12. EasyWarm

13. Control

14. Easywarm

15. EasyWarm

16. EasyWarm

17. EasyWarm

18. EasyWarm

19. EasyWarm

20. Control

21. Control

22. Control

23. EasyWarm

24. Control

25. Control

26. EasyWarm

27. EasyWarm

28. EasyWarm

29. EasyWarm

30. EasyWarm

31. Control

32. Control

33. EasyWarm

34. EasyWarm

35. Control

36. EasyWarm

37. EasyWarm

38. EasyWarm

39. EasyWarm

40. Control

41. Control

42. Control

43. Control

44. Control

45. EasyWarm

46. EasyWarm

47. EasyWarm

48. Control

49. Control

50. Control

51. EasyWarm

52. Control

53. EasyWarm

54. Control

55. Control

56. EasyWarm

57. EasyWarm

58. Control 
ANHANG

Seite 2 von 2

59. EasyWarm
60. EasyWarm
61. Control
62. Control
63. Control
64. EasyWarm
65. EasyWarm
66. Control
67. Control
68. Control
69. Control
70. Control
71. Control
72. EasyWarm
73. Control
74. EasyWarm
75. EasyWarm
76. Control
77. Control
78. EasyWarm
79. Control
80. EasyWarm

80 subjects randomized into 1 block

To reproduce this plan, use the seed 28116 


\section{Literaturverzeichnis}

Abelha FJ, Castro MA, Neves AM, Landeiro NM, Santos CC (2005):

Hypothermia in a surgical intensive care unit. BMC Anesthesiol $\underline{5}$, 7-16

Agrawal N, Sewell DA, Griswold ME, Frank SM, Hessel TW, Eisele DW (2003): Hypothermia during head and neck surgery. Laryngoscope $\underline{113}, 1278-1282$

Ammann G, Draxler H, Engler E, Germann PS, Germann R, Gottsauner-Wolf F, Greif R, Hiebl W, Huemer G, Illievich U (2004): Thermoregulation in der Anästhesie. CliniCum Sonderausgabe, 9-10 http://www.medizin-akademie.at/mm/konsensus-thermo.pdf

Andrzejowski J, Hoyle J, Eapen G, Turnbull D (2008): Effect of prewarming on post-induction core temperature and the incidence of inadvertent perioperative hypothermia in patients undergoing general anaesthesia. $\mathrm{Br} \mathrm{J}$ Anaesth $\underline{101}$, 627-631

Azzam FJ, Krock JL (1995): Thermal burns in two infants associated with a forced air warming system. Anesth Analg 81, 661

Bauer M, Bock M, Martin J, Schaper C, Chamaly M, Mahla E, Schlereth T, Max M, Hübner M (2007): Ungeplante postoperative Aufnahme elektiver Patienten auf Intensivstation: Eine prospektive Multi-Center-Analyse von Inzidenz, Kausalität und Vermeidbarkeit. Anästh Intensivmed 48: 542-550

Bay J, Nunn JF, Prys-Roberts C (1968): Factors influencing arterial PO2 during recovery from anaesthesia. $\mathrm{Br} \mathrm{J}$ Anaesth $\underline{40}, 398-407$

Bazett HC, Love L, et al. (1948): Temperature changes in blood flowing in arteries and veins in man. J Appl Physiol 1 , 3-19

Bennett J, Ramachandra V, Webster J, Carli F (1994): Prevention of hypothermia during hip surgery: effect of passive compared with active skin surface warming. Br J Anaesth $\underline{73}, 180-183$

Berti M, Casati A, Torri G, Aldegheri G, Lugani D, Fanelli G (1997): Active warming, not passive heat retention, maintains normothermia during combined epidural-general anesthesia for hip and knee arthroplasty. J Clin Anesth $\underline{9}$, 482486 
Bickler P, Sessler DI (1990): Efficiency of airway heat and moisture exchangers in anesthetized humans. Anesth Analg 71, 415-418

Bock M, Muller J, Bach A, Bohrer H, Martin E, Motsch J (1998): Effects of preinduction and intraoperative warming during major laparotomy. Br $\mathrm{J}$ Anaesth $\underline{80}, 159-163$

Brandes IF, Muller C, Perl T, Russo SG, Bauer M, Brauer A (2013): [Efficacy of a novel warming blanket : Prospective randomized trial]. Anaesthesist $\underline{62}, 137-$ 142

Brauer A, Weyland W (1998): Oesophageal heat exchanger in the prevention of perioperative hypothermia. Acta Anaesthesiol Scand 42, 1232-1233

Brauer A, Perl T, Wittkopp E, Braun U, Weyland W (2000): [Value of reflecting disposable insulation (Thermodrape) in preventing perioperative hypothermia]. Anasthesiol Intensivmed Notfallmed Schmerzther $\underline{35}$, 756-762

Brauer A, English MJ, Steinmetz N, Lorenz N, Perl T, Braun U, Weyland W (2002): Comparison of forced-air warming systems with upper body blankets using a copper manikin of the human body. Acta Anaesthesiol Scand 46, 965972

Brauer A, English MJ, Lorenz N, Steinmetz N, Perl T, Braun U, Weyland W (2003): Comparison of forced-air warming systems with lower body blankets using a copper manikin of the human body. Acta Anaesthesiol Scand 47, 58-64

Brauer A, Pacholik L, Perl T, English MJ, Weyland W, Braun U (2004a): Conductive heat exchange with a gel-coated circulating water mattress. Anesth Analg $\underline{99}$, 1742-1746

Brauer A, Pacholik L, Perl T, Mielck F, Weyland W, Braun U (2004b): [Heat transfer by conductive warming with circulating-water mattresses]. Anasthesiol Intensivmed Notfallmed Schmerzther $\underline{39}$, 471-476

Brauer A, Perl T, Uyanik Z, English MJ, Weyland W, Braun U (2004c): Perioperative thermal insulation: minimal clinically important differences? Br $\mathrm{J}$ Anaesth $\underline{\text { 92, 836-840 }}$

Brauer A, Perl T, Quintel M (2006): Perioperatives Wärmemanagement. Anaesthesist $\underline{55}, 1321-1340$ 
Brauer A, Perl T, English MJ, Quintel M (2007): Perioperative thermal insulation. Surg Technol Int 16, 41-45

Brauer A, Bovenschulte H, Perl T, Zink W, English MJM, Quintel M (2009): What determines the efficacy of forced-air systems? A manikin evaluation with upper body blankets. Anesth Analg 108, 192-198

Buhre W, Rossaint R (2003): Perioperative management and monitoring in anaesthesia. Lancet $\underline{362}, 1839-1846$

Camus Y, Delva E, Sessler DI, Lienhart A (1995): Pre-induction skin-surface warming minimizes intraoperative core hypothermia. J Clin Anesth $\underline{7}$, 384-388

Cobbe KA, Di Staso R, Duff J, Walker K, Draper N (2012): Preventing inadvertent hypothermia: comparing two protocols for preoperative forced-air warming. J Perianesth Nurs $\underline{27}$, 18-24

Crino MH, Nagel EL (1968): Thermal burns caused by warming blankets in the operating room. Anesthesiology $\underline{29}, 149-150$

de Brito Poveda V, Clark AM, Galvao CM (2013): A systematic review on the effectiveness of prewarming to prevent perioperative hypothermia. J Clin Nurs $\underline{22}, 906-918$

De Witte J, Sessler DI (2002): Perioperative shivering: physiology and pharmacology. Anesthesiology 96, 467-484

El-Gamal N, El-Kassabany N, Frank SM, Amar R, Khabar HA, El-Rahmany HK, Okasha AS (2000): Age-related thermoregulatory differences in a warm operating room environment (approximately 26 degrees $C$ ). Anesth Analg $\underline{90}$, 694-698

English MJ, Farmer C, Scott WA (1990): Heat loss in exposed volunteers. J Trauma $\underline{30}$, 422-425

English MJM (2001): Physical principles of heat transfer. Curr Anaesth Crit Care 12, 66-71 
Fossum S, Hays J, Henson MM (2001): A comparison study on the effects of prewarming patients in the outpatient surgery setting. J Perianesth Nurs $\underline{16}$, 187-194

Frank SM, Beattie C, Christopherson R, Norris EJ, Rock P, Parker S, Kimball AW, Jr. (1992): Epidural versus general anesthesia, ambient operating room temperature, and patient age as predictors of inadvertent hypothermia.

Anesthesiology $\underline{77}, 252-257$

Frank SM, Beattie C, Christopherson R, Norris EJ, Perler BA, Williams GM, Gottlieb SO (1993): Unintentional hypothermia is associated with postoperative myocardial ischemia. The Perioperative Ischemia Randomized Anesthesia Trial Study Group. Anesthesiology $\underline{78}$, 468-476

Frank SM, Fleisher LA, Olson KF, Gorman RB, Higgins MS, Breslow MJ, Sitzmann JV, Beattie C (1995 a): Multivariate determinants of early postoperative oxygen consumption in elderly patients. Effects of shivering, body temperature, and gender. Anesthesiology $\underline{83}, 241-249$

Frank SM, Higgins MS, Breslow MJ, Fleisher LA, Gorman RB, Randolph B, Sitzmann JV, Raff H, Beattle C (1995 b): The Catecholamine, Cortisol and Hemodynamic Responses to Mild Perioperative Hypothermia: A Randomized Clinical Trial. Anesthesiology $\underline{82}, 83-93$

Frank SM, Fleisher LA, Breslow MJ, Higgins MS, Olson KF, Kelly S, Beattie C (1997 a): Perioperative maintenance of normothermia reduces the incidence of morbid cardiac events. A randomized clinical trial. JAMA 277, 1127-1134

Frank SM, Higgins MS, Fleisher LA, Sitzmann JV, Raff H, Breslow MJ (1997 b): Adrenergic, respiratory, and cardiovascular effects of core cooling in humans. Am J Physiol 272, 557-562

Fritz HG, Holzmayr M, Walter B, Moeritz KU, Lupp A, Bauer R (2005): The effect of mild hypothermia on plasma fentanyl concentration and biotransformation in juvenile pigs. Anesth Analg $\underline{100}$, 996-1002

Gali B, Findlay JY, Plevak DJ (2003): Skin injury with the use of a water warming device. Anesthesiology $\underline{98}, 1509-1510$

Galvao CM, Marck PB, Sawada NO, Clark AM (2009): A systematic review of the effectiveness of cutaneous warming systems to prevent hypothermia. J Clin Nurs $\underline{18}, 627-636$ 
Galvao CM, Liang Y, Clark AM (2010): Effectiveness of cutaneous warming systems on temperature control: meta-analysis. J Adv Nurs $\underline{66}, 1196-1206$

Gautier H, Bonora M, Schultz SA, Remmers JE (1987): Hypoxia-induced changes in shivering and body temperature. J Appl Physiol $\underline{62}, 2477-2484$

Grocott HP, Mathew JP, Carver EH, Phillips-Bute B, Landolfo KP, Newman MF (2004): A randomized controlled trial of the Arctic Sun Temperature Management System versus conventional methods for preventing hypothermia during off-pump cardiac surgery. Anesth Analg $\underline{98}, 298-302$

Heier T, Caldwell JE, Sessler DI, Miller RD (1991): Mild intraoperative hypothermia increases duration of action and spontaneous recovery of vecuronium blockade during nitrous oxide-isoflurane anesthesia in humans. Anesthesiology $\underline{74}, 815-819$

Hocker J, Bein B, Bohm R, Steinfath M, Scholz J, Horn EP (2012): Correlation, accuracy, precision and practicability of perioperative measurement of sublingual temperature in comparison with tympanic membrane temperature in awake and anaesthetised patients. Eur J Anaesthesiol 29, 70-74

Hofer CK, Worn M, Tavakoli R, Sander L, Maloigne M, Klaghofer R, Zollinger A (2005): Influence of body core temperature on blood loss and transfusion requirements during off-pump coronary artery bypass grafting: a comparison of 3 warming systems. J Thorac Cardiovasc Surg $\underline{129}$, 838-843

Hohn DC, MacKay RD, Halliday B, Hunt TK (1976): Effect of O2 tension on microbicidal function of leukocytes in wounds and in vitro. Surg Forum 27, 1820

Hooper VD (2009): An introduction to the ASPAN evidence-based clinical practice guideline for the promotion of perioperative normothermia. $\mathrm{J}$ Perianesth Nurs $\underline{24}$, 269-270

Hooper VD, Chard R, Clifford T, Fetzer S, Fossum S, Godden B, Martinez EA, Noble KA, O'Brien D, Odom-Forren J (2009): ASPAN's evidence-based clinical practice guideline for the promotion of perioperative normothermia. J Perianesth Nurs 24, 271-287

Hopf HW, Hunt TK, West JM, Blomquist P, Goodson WH, 3rd, Jensen JA, Jonsson K, Paty PB, Rabkin JM, Upton RA (1997): Wound tissue oxygen 
tension predicts the risk of wound infection in surgical patients. Arch Surg $\underline{132}$, 997-1004

Horn EP, Werner C, Sessler DI, Steinfath M, Schulte am Esch J (1997): Late intraoperative clonidine administration prevents postanesthetic shivering after total intravenous or volatile anesthesia. Anesth Analg 84, 613-617

Horn EP, Sessler DI, Standl T, Schroeder F, Bartz HJ, Beyer JC, Schulte am Esch J (1998): Non-thermoregulatory shivering in patients recovering from isoflurane or desflurane anesthesia. Anesthesiology $\underline{89}$, 878-886

Horn EP, Schroeder F, Wilhelm S, Sessler DI, Standl T, von dem Busche K, Schulte am Esch J (1999): Postoperative pain facilitates nonthermoregulatory tremor. Anesthesiology $\underline{91}$, 979-984

Horn EP, Schroeder F, Gottschalk A, Sessler DI, Hiltmeyer N, Standl T, Schulte am Esch J (2002): Active warming during cesarean delivery. Anesth Analg 94, 409-414

Horn EP, Bein B, Bohm R, Steinfath M, Sahili N, Hocker J (2012): The effect of short time periods of pre-operative warming in the prevention of peri-operative hypothermia. Anaesthesia 67, 612-617

Huang JK, Shah EF, Vinodkumar N, Hegarty MA, Greatorex RA (2003): The Bair Hugger patient warming system in prolonged vascular surgery: an infection risk? Crit Care $\underline{7}, 13-16$

Hynson JM, Sessler DI (1992): Intraoperative warming therapies: a comparison of three devices. J Clin Anesth 4, 194-199

Hynson JM, Sessler DI, Moayeri A, McGuire J, Schroeder M (1993): The effects of preinduction warming on temperature and blood pressure during propofol/nitrous oxide anesthesia. Anesthesiology $\underline{79}$, 219-228

International Electrotechnical Commission (2009): Medical electrical equipmentPart 2-35: particular requirements for the basic safety and essential performance of heating devices using blankets, pads and mattresses and intended for heating in medical use. IEC80601-2-35, ed2.0 
Ikeda T, Sessler DI, Kikura M, Kazama T, Ikeda K, Sato S (1999): Less core hypothermia when anesthesia is induced with inhaled sevoflurane than with intravenous propofol. Anesth Analg $\underline{88}, 921-924$

Insler SR, O'Connor MS, Leventhal MJ, Nelson DR, Starr NJ (2000): Association between postoperative hypothermia and adverse outcome after coronary artery bypass surgery. Ann Thorac Surg $\underline{70}$, 175-181

Janicki PK, Higgins MS, Janssen J, Johnson RF, Beattie C (2001): Comparison of two different temperature maintenance strategies during open abdominal surgery: upper body forced-air warming versus whole body water garment. Anesthesiology $\underline{95}, 868-874$

Janicki PK, Stoica C, Chapman WC, Wright JK, Walker G, Pai R, Walia A, Pretorius M, Pinson CW (2002): Water warming garment versus forced air warming system in prevention of intraoperative hypothermia during liver transplantation: a randomized controlled trial. BMC Anesthesiol 2, 7-11

Johansson T, Lisander B, Ivarsson I (1999): Mild hypothermia does not increase blood loss during total hip arthroplasty. Acta Anaesthesiol Scand $\underline{43}$, $1005-1010$

Just B, Delva E, Camus Y, Lienhart A (1992): Oxygen uptake during recovery following naloxone. Relationship with intraoperative heat loss. Anesthesiology $\underline{76}, 60-64$

Just B, Trevien V, Delva E, Lienhart A (1993): Prevention of intraoperative hypothermia by preoperative skin-surface warming. Anesthesiology $\underline{79}, 214$ 218

Karalapillai D, Story D (2008): Hypothermia on arrival in the intensive care unit after surgery. Crit Care Resusc 10, 116-119

Karalapillai D, Story DA, Calzavacca P, Licari E, Liu YL, Hart GK (2009): Inadvertent hypothermia and mortality in postoperative intensive care patients: retrospective audit of 5050 patients. Anaesthesia $\underline{64}, 968-972$

Karalapillai D, Story D, Hart GK, Bailey M, Pilcher D, Schneider A, Kaufman M, Cooper DJ, Bellomo R (2013): Postoperative hypothermia and patient outcomes after major elective non-cardiac surgery. Anaesthesia $\underline{68}, 605-611$ 
Kim JY, Shinn H, Oh YJ, Hong YW, Kwak HJ, Kwak YL (2006): The effect of skin surface warming during anesthesia preparation on preventing redistribution hypothermia in the early operative period of off-pump coronary artery bypass surgery. Eur J Cardiothorac Surg 29, 343-347

Kongsayreepong S, Chaibundit C, Chadpaibool J, Komoltri C, Suraseranivongse S, Suwannanonda P, Raksamanee EO, Noocharoen P, Silapadech A, Parakkamodom S (2003): Predictor of core hypothermia and the surgical intensive care unit. Anesth Analg $\underline{96}, 826-833$

Kurz A, Go JC, Sessler DI, Kaer K, Larson MD, Bjorksten AR (1995a): Alfentanil slightly increases the sweating threshold and markedly reduces the vasoconstriction and shivering thresholds. Anesthesiology $\underline{83}, 293-299$

Kurz A, Sessler DI, Narzt E, Bekar A, Lenhardt R, Huemer G, Lackner F (1995b): Postoperative hemodynamic and thermoregulatory consequences of intraoperative core hypothermia. J Clin Anesth ㄱ, 359-366

Kurz A, Sessler DI, Lenhardt R (1996): Perioperative normothermia to reduce the incidence of surgical-wound infection and shorten hospitalization. Study of Wound Infection and Temperature Group. N Engl J Med 334, 1209-1215

Lamke LO, Nilsson GE, Reithner HL (1977): Water loss by evaporation from the abdominal cavity during surgery. Acta Chir Scand 143, 279-284

Leben J, Tryba M (1997): Prevention of hypothermia during surgery. Contribution of convective heating system and warm infusion. Ann N Y Acad Sci $\underline{813}, 807-811$

Lee L, Leslie K, Kayak E, Myles PS (2004): Intraoperative patient warming using radiant warming or forced-air warming during long operations. Anaesth Intensive Care $\underline{32}, 358-361$

Lefrant JY, Muller L, de La Coussaye JE, Benbabaali M, Lebris C, Zeitoun N, Mari C, Saissi G, Ripart J, Eledjam JJ (2003): Temperature measurement in intensive care patients: comparison of urinary bladder, oesophageal, rectal, axillary, and inguinal methods versus pulmonary artery core method. Intensive Care Med 29, 414-418

Lenhardt R, Marker E, Goll V, Tschernich H, Kurz A, Sessler DI, Narzt E, Lackner F (1997): Mild intraoperative hypothermia prolongs postanesthetic recovery. Anesthesiology $\underline{87}, 1318-1323$ 
Leslie K, Sessler DI, Bjorksten AR, Moayeri A (1995): Mild hypothermia alters propofol pharmacokinetics and increases the duration of action of atracurium. Anesth Analg $\underline{80}, 1007-1014$

Liu M, Hu X, Liu J (2001): The effect of hypothermia on isoflurane MAC in children. Anesthesiology $\underline{94}, 429-432$

Liu X, Dingley J, Elstad M, Scull-Brown E, Steen PA, Thoresen M (2013): Minimum alveolar concentration (MAC) for sevoflurane and xenon at normothermia and hypothermia in newborn pigs. Acta Anaesthesiol Scand $\underline{57}$, 646-653

Lopez M, Sessler DI, Walter K, Emerick T, Ozaki M (1994): Rate and gender dependence of the sweating, vasoconstriction, and shivering thresholds in humans. Anesthesiology $\underline{80}, 780-788$

Mahoney CB, Odom J (1999): Maintaining intraoperative normothermia: a meta-analysis of outcomes with costs. AANA J $\underline{67}, 155-163$

Matsukawa T, Kurz A, Sessler DI, Bjorksten AR, Merrifield B, Cheng C (1995 a): Propofol linearly reduces the vasoconstriction and shivering thresholds. Anesthesiology $\underline{82}, 1169-1180$

Matsukawa T, Sessler DI, Sessler AM, Schroeder M, Ozaki M, Kurz A, Cheng C (1995 b): Heat flow and distribution during induction of general anesthesia. Anesthesiology $\underline{82}, 662-673$

Matsuzaki Y, Matsukawa T, Ohki K, Yamamoto Y, Nakamura M, Oshibuchi T (2003): Warming by resistive heating maintains perioperative normothermia as well as forced air heating. Br J Anaesth 90, 689-691

Melling AC, Ali B, Scott EM, Leaper DJ (2001): Effects of preoperative warming on the incidence of wound infection after clean surgery: a randomised controlled trial. Lancet $\underline{358}, 876-880$

Moola S, Lockwood C (2011): Effectiveness of strategies for the management and/or prevention of hypothermia within the adult perioperative environment. Int $\mathrm{J}$ Evid Based Healthc $\underline{9}$, 337-345 
Morris $\mathrm{RH}$ (1971): Operating room temperature and the anesthetized, paralyzed patient. Arch Surg 102, 95-97

Ng SF, Oo CS, Loh KH, Lim PY, Chan YH, Ong BC (2003): A comparative study of three warming interventions to determine the most effective in maintaining perioperative normothermia. Anesth Analg 무, 171-176

NICE: National Institute for Health and Clinical Excellence (2008): Inadvertent perioperative hypothermia: Full Guideline. NICE Clinical Guideline 65,1-567 https://www.nice.org.uk/guidance/cg65

O'Brien D, Greenfield ML, Anderson JE, Smith BA, Morris M (2010): Comfort, satisfaction, and anxiolysis in surgical patients using a patient-adjustable comfort warming system: a prospective randomized clinical trial. J Perianesth Nurs $\underline{25}, 88-93$

Ozaki M, Sessler DI, Suzuki H, Ozaki K, Tsunoda C, Atarashi K (1995): Nitrous oxide decreases the threshold for vasoconstriction less than sevoflurane or isoflurane. Anesth Analg $\underline{80}, 1212-1216$

Patel N, Smith CE, Pinchak AC, Hagen JF (1996): Comparison of esophageal, tympanic, and forehead skin temperatures in adult patients. J Clin Anesth $\underline{8}$, 462-468

Perl T, Rhenius A, Eich CB, Quintel M, Heise D, Brauer A (2012): Conductive warming and insulation reduces perioperative hypothermia. Cent Eur J Med $\underline{7}$, 284-289

Rajagopalan S, Mascha E, Na J, Sessler DI (2008): The effects of mild perioperative hypothermia on blood loss and transfusion requirement. Anesthesiology $\underline{108}, 71-77$

Rasmussen YH, Leikersfeldt G, Drenck NE (1998): Forced-air surface warming versus oesophageal heat exchanger in the prevention of peroperative hypothermia. Acta Anaesthesiol Scand 42, 348-352

Rohrer MJ, Natale AM (1992): Effect of hypothermia on the coagulation cascade. Crit Care Med 20, 1402-1405

Sato H, Yamakage M, Okuyama K, Imai Y, Iwashita H, Ishiyama T, Matsukawa $\mathrm{T}$ (2008): Urinary bladder and oesophageal temperatures correlate better in 
patients with high rather than low urinary flow rates during non-cardiac surgery. Eur J Anaesthesiol 25, 805-809

Schmidt JH, Weyland W, Fritz U, Brauer A, Rathgeber J, Braun U (1996): [The comparative effectiveness of different infusion and blood warming methods]. Anaesthesist $\underline{45}, 1067-1074$

Schmied H, Kurz A, Sessler DI, Kozek S, Reiter A (1996): Mild hypothermia increases blood loss and transfusion requirements during total hip arthroplasty. Lancet $\underline{347}$, 289-292

Sellden E, Lindahl SG (1998): Postoperative nitrogen excretion after amino acid-induced thermogenesis under anesthesia. Anesth Analg 87, 641-646

Sellden E, Branstrom R, Brundin T (1996): Preoperative infusion of amino acids prevents postoperative hypothermia. Br J Anaesth $\underline{76}$, 227-234

Sessler DI (1997): Mild perioperative hypothermia. N Engl J Med 336, 17301737

Sessler DI (2000): Perioperative heat balance. Anesthesiology 92, 578-596

Sessler DI (2001): Complications and treatment of mild hypothermia. Anesthesiology $\underline{95}, 531-543$

Sessler DI (2008): Temperature monitoring and perioperative thermoregulation. Anesthesiology 109, 318-338

Sessler DI (2009): Thermoregulatory defense mechanisms. Crit Care Med $\underline{37}$, 203-210

Sessler DI, Moayeri A (1990): Skin-surface warming: heat flux and central temperature. Anesthesiology $\underline{73}, 218-224$

Sessler DI, Schroeder M (1993): Heat loss in humans covered with cotton hospital blankets. Anesth Analg $\underline{77}, 73-77$

Sessler DI, McGuire J, Sessler AM (1991a): Perioperative thermal insulation. Anesthesiology $\underline{74}, 875-879$ 
Sessler DI, Rubinstein EH, Moayeri A (1991b): Physiologic responses to mild perianesthetic hypothermia in humans. Anesthesiology $\underline{75}, 594-610$

Sessler DI, Schroeder M, Merrifield B, Matsukawa T, Cheng C (1995): Optimal duration and temperature of prewarming. Anesthesiology $\underline{82}, 674-681$

Sharp RJ, Chesworth T, Fern ED (2002): Do warming blankets increase bacterial counts in the operating field in a laminar-flow theatre? J Bone Joint Surg Br $\underline{84}$, 486-488

Sheffield CW, Sessler DI, Hopf HW, Schroeder M, Moayeri A, Hunt TK, West JM (1996): Centrally and locally mediated thermoregulatory responses alter subcutaneous oxygen tension. Wound Repair Regen 4, 339-345

Slotman GJ, Jed EH, Burchard KW (1985): Adverse effects of hypothermia in postoperative patients. Am J Surg $\underline{149}$, 495-501

Smeulers NJ, Wierda JM, van den Broek L, Gallandat Huet RC, Hennis PJ (1995): Hypothermic cardiopulmonary bypass influences the concentrationresponse relationship and the biodisposition of rocuronium. Eur $\mathrm{J}$ Anaesthesiol Suppl 11, 91-94

Smith CE, Desai R, Glorioso V, Cooper A, Pinchak AC, Hagen KF (1998): Preventing hypothermia: convective and intravenous fluid warming versus convective warming alone. J Clin Anesth 10, 380-385

Sumer BD, Myers LL, Leach J, Truelson JM (2009): Correlation between intraoperative hypothermia and perioperative morbidity in patients with head and neck cancer. Arch Otolaryngol Head Neck Surg 135, 682-686

Taguchi A, Ratnaraj J, Kabon B, Sharma N, Lenhardt R, Sessler DI, Kurz A (2004): Effects of a circulating-water garment and forced-air warming on body heat content and core temperature. Anesthesiology 100, 1058-1064

The Commission for Thermal Physiology of the International Union of Physiological Sciences (2001): Glossary of terms for thermal physiology, 3rd edn. Jpn J Physiol 1ㅡ, 245-280 
Torossian A (2008): Thermal management during anaesthesia and thermoregulation standards for the prevention of inadvertent perioperative hypothermia. Best Pract Res Clin Anaesthesiol 22, 659-668

Truell KD, Bakerman PR, Teodori MF, Maze A (2000): Third-degree burns due to intraoperative use of a Bair Hugger warming device. Ann Thorac Surg $\underline{69}$, 1933-1934

Valeri CR, Feingold H, Cassidy G, Ragno G, Khuri S, Altschule MD (1987): Hypothermia-induced reversible platelet dysfunction. Ann Surg 205, 175-181

Valeri CR, Khabbaz K, Khuri SF, Marquardt C, Ragno G, Feingold H, Gray AD, Axford T (1992): Effect of skin temperature on platelet function in patients undergoing extracorporeal bypass. J Thorac Cardiovasc Surg 104, 108-116

van Oss CJ, Absolom DR, Moore LL, Park BH, Humbert JR (1980): Effect of temperature on the chemotaxis, phagocytic engulfment, digestion and $\mathrm{O} 2$ consumption of human polymorphonuclear leukocytes. J Reticuloendothel Soc $\underline{27}, 561-565$

Vanni SM, Braz JR, Modolo NS, Amorim RB, Rodrigues GR, Jr. (2003): Preoperative combined with intraoperative skin-surface warming avoids hypothermia caused by general anesthesia and surgery. J Clin Anesth 15, 119125

Wenisch C, Narzt E, Sessler DI, Parschalk B, Lenhardt R, Kurz A, Graninger W (1996): Mild intraoperative hypothermia reduces production of reactive oxygen intermediates by polymorphonuclear leukocytes. Anesth Analg ⒉ 810-816

Winkler M, Akca O, Birkenberg B, Hetz H, Scheck T, Arkilic CF, Kabon B, Marker E, Grubl A, Czepan R (2000): Aggressive warming reduces blood loss during hip arthroplasty. Anesth Analg 91, 978-984

Wong A, Walker S, Bradley M (2004): Comparison of a radiant patient warming device with forced air warming during laparoscopic cholecystectomy. Anaesth Intensive Care 32, 93-99

Wong PF, Kumar S, Bohra A, Whetter D, Leaper DJ (2007): Randomized clinical trial of perioperative systemic warming in major elective abdominal surgery. Br J Surg 94, 421-426 
Zink RS, laizzo PA (1993): Convective warming therapy does not increase the risk of wound contamination in the operating room. Anesth Analg $\underline{76}, 50-53$

Internetquellen:

Gebrauchsanweisung Barrier® Easywarm®, Mönlycke Healthcare GmbH, Erkrath, Deutschland 2012, Seitenabruf am 01.09.2014:

http://www.molnlycke.de/Documents/DEU/Surgical/EasyWarm/DE BARRIER EasyWarm Produ ctSheet\%20291113.pdf

http://www.molnlycke.de/Documents/DEU/Surgical/EasyWarm/DE\%20BARRIER EasyWarm Po ster A3 v1 291113.pdf

http://www.molnlycke.de/patientenwarmemanagement/easywarm/ Seitenabruf am 01.09.2014 


\section{Danksagung}

Mein besonderer Dank gilt meinem Doktorvater Herrn Prof. Dr. med. Anselm Bräuer und Betreuer Herrn Dr. med. Ivo Brandes für die Möglichkeit, diese klinisch-praktische Arbeit durchführen zu können sowie für die außergewöhnlich gute Betreuung der Doktorarbeit.

Danken möchte ich auch Herrn Prof. Dr. Dr. med. Martin Bauer, der mir die Durchführung dieses Projektes ermöglichte.

Ebenfalls danke ich dem Pflegeteam in der Wartezone, im Aufwachraum und den HNO-Stationen sowie den HNO-chirurgischen Kollegen für ihr Interesse und die Kooperation bei der Durchführung dieses Projektes. 